\title{
Optimality of Affine Policies in Multistage Robust Optimization
}

\author{
Dimitris Bertsimas \\ Sloan School of Management and Operations Research Center, Massachusetts Institute of Technology, \\ Cambridge, Massachusetts 02139, dbertsim@mit.edu, http://www.mit.edu/ dbertsim/
}

Dan A. Iancu

Operations Research Center, Massachusetts Institute of Technology, Cambridge, Massachusetts 02139, daniancu@ mit.edu, http://www.mit.edu/ daniancu/

\section{Pablo A. Parrilo}

Laboratory for Information and Decision Systems, Massachusetts Institute of Technology, Cambridge, Massachusetts 02139, parrilo@ mit.edu, http://www.mit.edu/ parrilo/

\begin{abstract}
In this paper, we prove the optimality of disturbance-affine control policies in the context of one-dimensional, constrained, multistage robust optimization. Our results cover the finite-horizon case, with minimax (worst-case) objective, and convex state costs plus linear control costs. We develop a new proof methodology, which explores the relationship between the geometrical properties of the feasible set of solutions and the structure of the objective function. Apart from providing an elegant and conceptually simple proof technique, the approach also entails very fast algorithms for the case of piecewise-affine state costs, which we explore in connection with a classical inventory management application.

Key words: robust optimization; multistage minimax; optimal policies; convex costs; dynamic; programming; inventory management

MSC2000 subject classification: Primary: 90C47, 49J30; secondary: 90C25, 90C39

OR/MS subject classification: Primary: dynamic programming/optimal control—Markov, finite state and infinite state, mathematics-Convexity; secondary: mathematics-Piecewise linear, inventory

History: Received January 4, 2009; revised November 16, 2009, and February 8, 2010. Published online in Articles in Advance April 30, 2010.
\end{abstract}

1. Introduction. Multistage optimization problems under uncertainty have been prevalent in numerous fields of science and engineering, and have elicited interest from diverse research communities, on both a theoretical and a practical level. Several solution approaches have been proposed, with various degrees of generality, tractability, and performance guarantees. Some of the most successful ones include exact and approximate dynamic programming (DP), stochastic programming, sampling-based methods, and more recently, robust and adaptive optimization, which is the focus of this paper.

The topics of robust optimization and robust control have been studied, under different names, by a variety of academic groups, mostly in operations research (Ben-Tal and Nemirovski [2, 3]; Ben-Tal et al. [6]; Bertsimas and Sim [12, 13]; Bertsimas et al. [14]) and control theory (Bertsekas and Rhodes [10], Fan et al. [19], El-Ghaoui et al. [18], Grieder et al. [22], Bemporad et al. [1], Kerrigan and Maciejowski [25], Zhou and Doyle [33], Dullerud and Paganini[17]), with considerable effort put into justifying the assumptions and general modeling philosophy. As such, the goal of this paper is not to motivate the use of robust (and, more generally, distribution free) techniques. Rather, we take the modeling approach as a given, and investigate tractability and performance issues in the context of a certain class of optimization problems. More precisely, we are concerned with the following multistage decision problem.

Problem 1. Consider a one-dimensional, discrete-time, linear dynamical system

$$
x_{k+1}=\alpha_{k} \cdot x_{k}+\beta_{k} \cdot u_{k}+\gamma_{k} \cdot w_{k},
$$

where $\alpha_{k}, \beta_{k}, \gamma_{k} \neq 0$ are known scalars, and the initial state $x_{1} \in \mathbb{R}$ is specified. The random disturbances $w_{k}$ are unknown, but bounded

$$
w_{k} \in \mathscr{W}_{k} \stackrel{\text { def }}{=}\left[\underline{w}_{k}, \bar{w}_{k}\right] \text {. }
$$

We would like to find a sequence of robust controllers $\left\{u_{k}\right\}$, obeying upper-and lower-bound constraints

$$
u_{k} \in\left[L_{k}, U_{k}\right]
$$

$\left(L_{k}, U_{k} \in \mathbb{R}\right.$ are known and fixed) and minimizing the following cost function over a finite horizon $1, \ldots, T$,

$$
J=c_{1} u_{1}+\max _{w_{1}}\left[h_{1}\left(x_{2}\right)+c_{2} u_{2}+\max _{w_{2}}\left[h_{2}\left(x_{3}\right)+\cdots+\max _{w_{T-1}}\left[c_{T} u_{T}+\max _{w_{T}} h_{T}\left(x_{T+1}\right)\right] \cdots\right]\right],
$$

where the functions $h_{k}: \mathbb{R} \rightarrow \mathbb{R} \cup\{+\infty\}$ are extended real and convex, and $c_{k} \geq 0$ are fixed and known. 
The problem corresponds to a situation in which, at every time step $k$, the decision maker has to compute a control action $u_{k}$, in such a way that certain constraints (3) are obeyed, and a cost penalizing both the state $\left(h_{k}\left(x_{k+1}\right)\right)$ and the control $\left(c_{k} \cdot u_{k}\right)$ is minimized. The uncertainty, $w_{k}$, always acts so as to maximize the costs, hence the problem solved by the decision maker corresponds to a worst-case scenario (a minimization of the maximum possible cost). An example of such a problem, which we use extensively in this paper, is the following.

EXAmple 1.1. Consider a retailer selling a single product over a planning horizon $1, \ldots, T$. The demands $w_{k}$ from customers are only known to be bounded, and the retailer can replenish her inventory $x_{k}$ by placing capacitated orders $u_{k}$, at the beginning of each period, for a cost of $c_{k}$ per unit of product. After the demand $w_{k}$ is realized, the retailer incurs holding costs $H_{k} \cdot \max \left\{0, x_{k}+u_{k}-w_{k}\right\}$ for all the amounts of supply stored on her premises, and penalties $B_{k} \cdot \max \left\{w_{k}-x_{k}-u_{k}, 0\right\}$, for any demand that is backlogged.

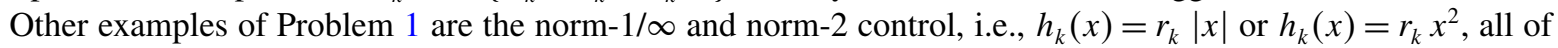
which have been studied extensively in the control literature in the unconstrained case (see Zhou and Doyle [33], Dullerud and Paganini [17]).

The solution to Problem 1 could be obtained using a "classical" DP formulation (Bertsekas [9]), in which the optimal policies $u_{k}^{\star}\left(x_{k}\right)$ and the optimal value functions $J_{k}^{\star}\left(x_{k}\right)$ are computed backward in time, starting at the end of the planning horizon, $k=T$. The resulting policies are piecewise affine in the states $x_{k}$, and have properties that are well known and documented in the literature (e.g., for the inventory model above, they exactly correspond to the base-stock ordering policies of Scarf et al. [31] and Kasugai and Kasegai [23]). We remark that the piecewise structure is essential, i.e., control policies that are only affine in the states $x_{k}$ are, in general, suboptimal.

In this paper, we would like to study the performance of a new class of policies, where instead of regarding the controllers $u_{k}$ as functions of the state $x_{k}$, one seeks disturbance-feedback policies, i.e., policies that are directly parameterizations in the observed disturbances:

$$
u_{k}: \mathscr{W}_{1} \times \mathscr{W}_{2} \times \cdots \times \mathscr{W}_{k-1} \rightarrow \mathbb{R}
$$

One such example (of particular interest) is the disturbance-affine class, i.e., policies of the form (5), which are also affine. In this new framework, we require that constraint (3) should be robustly feasible, i.e.,

$$
u_{k}(\mathbf{w}) \in\left[L_{k}, U_{k}\right], \quad \forall \mathbf{w} \in \mathscr{W}_{1} \times \cdots \times \mathscr{W}_{k-1} .
$$

Note that if we insisted on this category of parameterizations, then we would have to consider a new state for the system, $\mathbf{X}_{k}$, which would include at least all the past observed disturbances, as well as possibly other information (e.g., the previous controls $\left\{u_{t}\right\}_{1 \leq t<k}$, the previous states $\left\{x_{t}\right\}_{1 \leq t<k}$, or some combination thereof). Compared with the original, compact state formulation, $x_{k}$, the new state $\mathbf{X}_{k}$ would become much larger, and solving the DP with state variable $\mathbf{X}_{k}$ would produce exactly the same optimal objective function value. Therefore, one should rightfully ask what the benefit for introducing such a complicated state might be.

The hope is that, by considering policies over a larger state, simpler functional forms might be sufficient for optimality, for instance, affine policies. These have a very compact representation, because only the coefficients of the parameterization are needed, and for certain classes of convex costs $h_{k}(\cdot)$, there may be efficient procedures available for computing them.

This approach is also not new in the literature. It has been originally advocated in the context of stochastic programming (see Charnes et al. [15], Garstka and Wets [20], and references therein), where such policies are known as decision rules. More recently, the idea has received renewed interest in robust optimization (Ben-Tal et al. [7]), and has been extended to linear systems theory (Ben-Tal et al. [4, 5]), with notable contributions from researchers in robust model predictive control and receding horizon control (see Löfberg [27], Bemporad et al. [1], Kerrigan and Maciejowski [25], Skaf and Boyd [32], and references therein). In all the papers, which usually deal with the more general case of multidimensional linear systems, the authors typically restrict attention, for purposes of tractability, to the class of disturbance-affine policies, and show how the corresponding policy parameters can be found by solving specific types of optimization problems, which vary from linear and quadratic programs (Ben-Tal et al. [4], Kerrigan and Maciejowski [24, 25]) to conic and semidefinite (Löfberg [27], Ben-Tal et al. [4]), or even multiparametric, linear, or quadratic programs (Bemporad et al. [1]). The tractability and empirical success of disturbance-affine policies in the robust framework have lead to their reexamination in stochastic settings, with several recent papers (Nemirovski and Shapiro [29], Chen et al. [16], Kuhn et al. [26]) providing tractable methods for determining the best parameters of the policies, in the context of both single-stage and multistage linear stochastic programming problems. 
The first steps toward analyzing the properties of such parameterizations were made in Kerrigan and Maciejowski [25], where the authors show that, under suitable conditions, the resulting affine parameterization has certain desirable system-theoretic properties (stability and robust invariance). Other notable contributions were Goulart and Kerrigan [21] and Ben-Tal et al. [4], who prove that the class of affine disturbance-feedback policies is equivalent to the class of affine state feedback policies with memory of prior states, thus subsuming the well-known classes of open-loop and prestabilizing control policies. In terms of characterizing the optimal objective obtained by using affine parameterizations, most research efforts thus far focus on providing tractable dual formulations, which allow a computation of lower or upper bounds to the problems, and hence an assessment of the degree of suboptimality (see Kuhn et al. [26] for details). Empirically, several authors have observed that affine policies deliver excellent performance, with Ben-Tal et al. [8] and Kuhn et al. [26] reporting many instances in which they are actually optimal. However, to the best of our knowledge, apart from these advances, there has been very little progress in proving results about the quality of the objective function value resulting from the use of such parameterizations.

Our main result, summarized in Theorem 3.1 of $\S 3$, is that, for Problem 1 stated above, disturbance-affine policies of the form (5) are optimal. Furthermore, we prove that a certain (affine) relaxation of the state costs is also possible, without any loss of optimality, which gives rise to very efficient algorithms for computing the optimal affine policies when the state costs $h_{k}(\cdot)$ are piecewise affine. To the best of our knowledge, this is the first result of its kind, and it is surprising, particularly because similar policies, i.e., decision rules, are known to be severely suboptimal for stochastic problems (see, e.g., Garstka and Wets [20], and our discussion in §4.5). The result provides intuition and motivation for the widespread advocation of such policies in both theory and applications. Our theoretical constructions are tight, i.e., if the conditions in Problem 1 are slightly perturbed, then simple counterexamples for Theorem $3.1 \mathrm{can}$ be found (see $\$ 4.5$ ). The proof of the theorem itself is atypical, consisting of a forward induction and making use of polyhedral geometry to construct the optimal affine policies. Thus we gain insight into the structure and properties of these policies, which we explore in connection with the inventory management problem in Example 1.1.

We remark that two concepts are central to our constructions. First, considering policies over an enlarged state space (here, the history of all disturbances) is essential, in the sense that affine state-feedback controllers depending only on the current state $x_{k}$ (e.g., $\left.u_{k}\left(x_{k}\right)=\ell_{k} x_{k}+\ell_{k, 0}\right)$ are, in general, suboptimal for the problems we consider. Second, the construction makes full use of the fact that the problem objective is of minimax type, which allows the decision maker the freedom of computing policies that are not optimal in every state of the system evolution (but rather, only in states that could result in worst-case outcomes). This is a fundamental distinction between robust and stochastic models for decision making under uncertainty, and it suggests that utilizing the framework of DP to solve multiperiod robust problems might be an unnecessary overkill, since simpler (not necessarily "Bellman optimal") policies might be sufficient to achieve the optimal worst-case outcome.

The paper is organized as follows. Section 2 presents an overview of the DP formulation in state variable $x_{k}$, extracting the optimal policies $u_{k}^{\star}\left(x_{k}\right)$ and optimal value functions $J_{k}^{\star}\left(x_{k}\right)$, as well as some of their properties. Section 3 contains our main result, and briefly discusses some immediate extensions and computational implications. In $\S 4$, we introduce the constructive proof for building the affine control policies and the affine cost relaxations, and present counterexamples that prevent a generalization of the results. In $\S 5$, we discuss our results in connection with the classical inventory management problem of Example 1.1. Section 6 presents our conclusions and directions for future research.

1.1. Notation. Throughout the rest of the paper, the subscripts $k$ and $t$ are used to denote time dependency, and vector quantities are distinguished by boldface symbols, with optimal quantities having a $\star$ superscript, e.g., $J_{k}^{\star}$. Also, $\overline{\mathbb{R}}=\mathbb{R} \cup\{+\infty\}$ stands for the set of extended reals.

Because we seek policies parameterized directly in the uncertainties, we introduce $\mathbf{w}_{k} \stackrel{\text { def }}{=}\left(w_{1}, \ldots, w_{k-1}\right)$ to denote the history of known disturbances in period $k$, and $\mathscr{H}_{k} \stackrel{\text { def }}{=} \mathscr{W}_{1} \times \cdots \times \mathscr{W}_{k-1}$ to denote the corresponding uncertainty set (a hypercube in $\mathbb{R}^{k-1}$ ). A function $q_{k}$ that depends affinely on variables $w_{1}, \ldots, w_{k-1}$ is denoted by $q_{k}\left(\mathbf{w}_{k}\right) \stackrel{\text { def }}{=} q_{k, 0}+\mathbf{q}_{k}^{\prime} \mathbf{w}_{k}$, where $\mathbf{q}_{k}$ is the vector of coefficients, and ' denotes the usual transpose.

2. DP solution. As mentioned in the introduction, the solution to Problem 1 can be obtained using a "classical" DP formulation (see, e.g., Bertsekas [9]), in which the state is taken to be $x_{k}$, and the optimal policies $u_{k}^{\star}\left(x_{k}\right)$ and optimal value functions $J_{k}^{\star}\left(x_{k}\right)$ are computed starting at the end of the planning horizon, $k=T$, and moving backward in time. In this section, we briefly outline the DP solution for our problem, and state some of 
the key properties that are used throughout the rest of the paper. For completeness, a full proof of the results is included in $\S \mathrm{A} 1$ of the appendix.

To simplify the notation, we remark that, since the constraints on the controls $u_{k}$ and the bounds on the disturbances $w_{k}$ are time varying, and independent for different time periods, we can restrict attention without loss of generality, ${ }^{1}$ to a system with $\alpha_{k}=\beta_{k}=\gamma_{k}=1$. With this simplification, the problem that we would like to solve is the following:

$$
\min _{u_{1}}\left[c_{1} u_{1}+\max _{w_{1}}\left[h_{1}\left(x_{2}\right)+\cdots+\min _{u_{k}}\left[c_{k} u_{k}+\max _{w_{k}}\left[h_{k}\left(x_{k+1}\right)+\cdots+\min _{u_{T}}\left[c_{T} u_{T}+\max _{w_{T}} h_{T}\left(x_{T+1}\right)\right] \cdots\right]\right],\right.\right.
$$

$$
\begin{array}{ll}
\text { s.t. } & x_{k+1}=x_{k}+u_{k}+w_{k}, \\
& L_{k} \leq u_{k} \leq U_{k} \quad \forall k \in\{1,2, \ldots, T\}, \\
& w_{k} \in \mathscr{W}_{k}=\left[\underline{w}_{k}, \bar{w}_{k}\right] .
\end{array}
$$

The corresponding Bellman recursion for $(D P)$ can then be written as follows:

$$
J_{k}^{\star}\left(x_{k}\right) \stackrel{\text { def }}{=} \min _{L_{k} \leq u_{k} \leq U_{k}}\left[c_{k} u_{k}+\max _{w_{k} \in \mathscr{W}_{k}}\left[h_{k}\left(x_{k}+u_{k}+w_{k}\right)+J_{k+1}^{\star}\left(x_{k}+u_{k}+w_{k}\right)\right]\right],
$$

where $J_{T+1}^{\star}\left(x_{T+1}\right) \equiv 0$. By defining

$$
\begin{gathered}
y_{k} \stackrel{\text { def }}{=} x_{k}+u_{k}, \\
g_{k}\left(y_{k}\right) \stackrel{\text { def }}{=} \max _{w_{k} \in \mathscr{V}_{k}}\left[h_{k}\left(y_{k}+w_{k}\right)+J_{k+1}^{\star}\left(y_{k}+w_{k}\right)\right],
\end{gathered}
$$

we obtain the following solution to the Bellman recursion (see $\S \mathrm{A} 1$ in the appendix for the derivation):

$$
\begin{gathered}
u_{k}^{\star}\left(x_{k}\right)= \begin{cases}U_{k} & \text { if } x_{k}<y_{k}^{\star}-U_{k}, \\
-x_{k}+y_{k}^{\star} & \text { otherwise, } \\
L_{k} & \text { if } x_{k}>y_{k}^{\star}-L_{k},\end{cases} \\
J_{k}^{\star}\left(x_{k}\right)=c_{k} \cdot u_{k}^{\star}\left(x_{k}\right)+g_{k}\left(x_{k}+u_{k}^{\star}\left(x_{k}\right)\right)= \begin{cases}c_{k} \cdot U_{k}+g_{k}\left(x_{k}+U_{k}\right) & \text { if } x_{k}<y_{k}^{\star}-U_{k}, \\
c_{k} \cdot\left(y_{k}^{\star}-x_{k}\right)+g_{k}\left(y^{\star}\right) & \text { otherwise, } \\
c_{k} \cdot L_{k}+g_{k}\left(x_{k}+L_{k}\right) & \text { if } x_{k}>y_{k}^{\star}-L_{k},\end{cases}
\end{gathered}
$$

where $y_{k}^{\star}$ represents the minimizer ${ }^{2}$ of the convex function $c_{k} \cdot y+g_{k}(y)$ (for the inventory Example 1.1, $y_{k}^{\star}$ is the base-stock level in period $k$, i.e., the inventory position just after ordering and before seeing the demand). A typical example of the optimal control law and the optimal value function is shown in Figure 1.

The main properties of the solution relevant for our later treatment are listed as follows:

[P1] The optimal control law $u_{k}^{\star}\left(x_{k}\right)$ is piecewise affine, continuous, and nonincreasing.

[P2] The optimal value function $J_{k}^{\star}\left(x_{k}\right)$ and the function $g_{k}\left(y_{k}\right)$ are convex.

[P3] The difference in the values of the optimal control law at two distinct arguments $s \leq t$ always satisfies $0 \leq u_{k}^{\star}(s)-u_{k}^{\star}(t) \leq t-s$. Equivalently, $x_{k}+u_{k}^{\star}\left(x_{k}\right)$ is nondecreasing as a function of $x_{k}$.

3. Optimality of affine policies in $\mathbf{w}_{k}$. In this section, we introduce our main contribution; namely, a proof that policies that are affine in the disturbances $\mathbf{w}_{k}$ are, in fact, optimal for problem $(D P)$. Using the same notation as in $\S 2$, and with $J_{1}^{\star}\left(x_{1}\right)$ denoting the optimal overall value, we can summarize our main result in the following theorem.

\footnotetext{
${ }^{1}$ Such a system can always be obtained by the linear change of variables $\tilde{x}_{k}=x_{k} / \prod_{i=1}^{k-1} \alpha_{i}$, and by suitably scaling the bounds $L_{k}, U_{k}, \underline{w}_{k}, \bar{w}_{k}$. ${ }^{2}$ For simplicity of exposition, we work under the assumption that the minimizer is unique. The results can be extended to the case of multiple minimizers.
} 


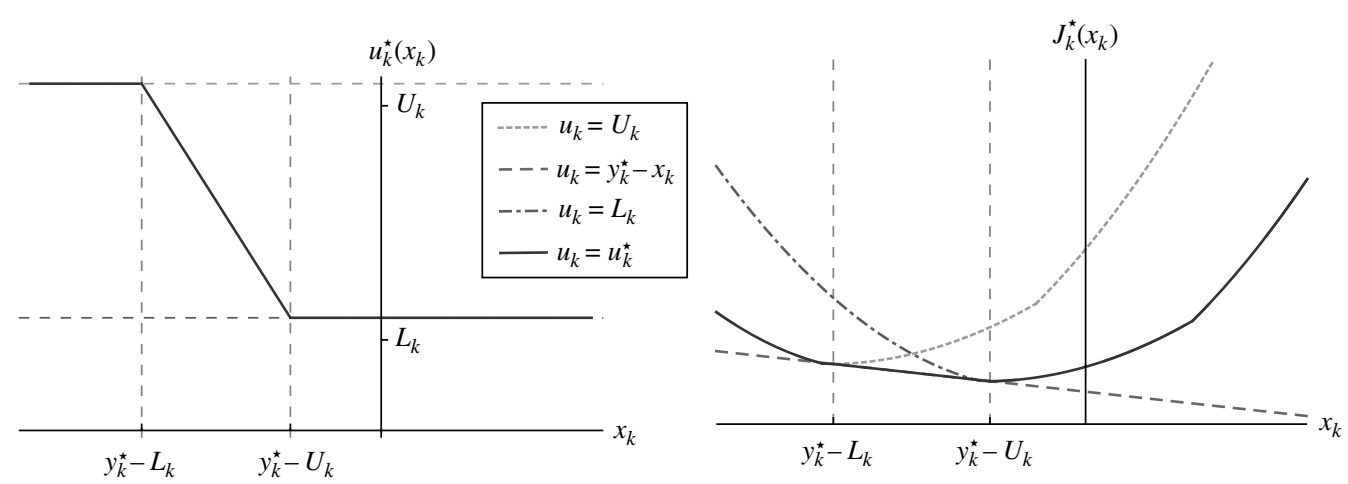

FIGURE 1. Optimal control law $u_{k}^{\star}\left(x_{k}\right)$ and optimal value function $J_{k}^{\star}\left(x_{k}\right)$ at time $k$.

Theorem 3.1 (Optimality of Disturbance-Affine Policies). Affine disturbance-feedback policies are optimal for Problem 1 stated in the introduction. More precisely, for every time step $k=1, \ldots, T$, the following quantities exist:

$$
\begin{aligned}
& \text { an affine control policy, } q_{k}\left(\mathbf{w}_{k}\right) \stackrel{\text { def }}{=} q_{k, 0}+\mathbf{q}_{k}^{\prime} \mathbf{w}_{k} \text {, } \\
& \text { an affine running cost, } z_{k}\left(\mathbf{w}_{k+1}\right) \stackrel{\text { def }}{=} z_{k, 0}+\mathbf{z}_{k}^{\prime} \mathbf{w}_{k+1} \text {, }
\end{aligned}
$$

such that the following properties are obeyed:

$$
\begin{gathered}
L_{k} \leq q_{k}\left(\mathbf{w}_{k}\right) \leq U_{k}, \quad \forall \mathbf{w}_{k} \in \mathscr{H}_{k}, \\
z_{k}\left(\mathbf{w}_{k+1}\right) \geq h_{k}\left(x_{1}+\sum_{t=1}^{k}\left(q_{t}\left(\mathbf{w}_{t}\right)+w_{t}\right)\right), \quad \forall \mathbf{w}_{k+1} \in \mathscr{H}_{k+1}, \\
J_{1}^{\star}\left(x_{1}\right)=\max _{w_{k+1} \in \mathscr{H}_{k+1}}\left[\sum_{t=1}^{k}\left(c_{t} \cdot q_{t}\left(\mathbf{w}_{t}\right)+z_{t}\left(\mathbf{w}_{t+1}\right)\right)+J_{k+1}^{\star}\left(x_{1}+\sum_{t=1}^{k}\left(q_{t}\left(\mathbf{w}_{t}\right)+w_{t}\right)\right)\right] .
\end{gathered}
$$

Let us interpret the main statements in the theorem. Equation (11a) confirms the existence of an affine policy $q_{k}\left(\mathbf{w}_{k}\right)$ that is robustly feasible, i.e., that obeys the control constraints, no matter what the realization of the disturbances may be. Equation (11b) states the existence of an affine cost $z_{k}\left(\mathbf{w}_{k+1}\right)$ that is always larger than the convex state cost $h_{k}\left(x_{k+1}\right)$ incurred when the affine policies $\left\{q_{t}(\cdot)\right\}_{1 \leq t \leq k}$ are used. Equation (11c) guarantees that, despite using the (suboptimal) affine control law $q_{k}(\cdot)$ and incurring a (potentially larger) affine stage cost $z_{k}(\cdot)$, the overall objective function value $J_{1}^{\star}\left(x_{1}\right)$ is, in fact, not increased. This translates in the following two main results:

- Existential result. Affine policies $q_{k}\left(\mathbf{w}_{k}\right)$ are, in fact, optimal for Problem 1.

- Computational result. When the convex costs $h_{k}\left(x_{k+1}\right)$ are piecewise affine, the optimal affine policies $\left\{q_{k}\left(\mathbf{w}_{k}\right)\right\}_{1 \leq k \leq T}$ can be computed by solving a linear programming problem.

To see why the second implication would hold, suppose that $h_{k}\left(x_{k+1}\right)$ is the maximum of $m_{k}$ affine functions, $h_{k}\left(x_{k+1}\right)=\max \left(p_{k}^{i} \cdot x_{k+1}+p_{k, 0}^{i}\right), i \in\left\{1, \ldots, m_{k}\right\}$. Then, the optimal affine policies $q_{k}\left(\mathbf{w}_{k}\right)$ can be obtained by solving the following optimization problem (see Ben-Tal et al. [8]):

$(A A R C)$

$$
\begin{aligned}
& \min _{J ;\left\{q_{k, t}\right\} ;\left\{z_{k, t}\right\}} J \\
& \text { s.t. } \quad \forall \mathbf{w} \in \mathscr{H}_{T+1}, \quad \forall k \in\{1, \ldots, T\}: \\
& \quad J \geq \sum_{k=1}^{T}\left[c_{k} \cdot q_{k, 0}+z_{k, 0}+\sum_{t=1}^{k-1}\left(c_{t} \cdot q_{k, t}+z_{k, t}\right) \cdot w_{t}+z_{k, k} \cdot w_{k}\right], \\
& z_{k, 0}+\sum_{t=1}^{k} z_{k, t} \cdot w_{t} \geq p_{k}^{i} \cdot\left[x_{1}+\sum_{t=1}^{k}\left(q_{t, 0}+\sum_{\tau=1}^{t-1} q_{t, \tau} \cdot w_{\tau}+w_{t}\right)\right]+p_{k, 0}^{i} \quad \forall i \in\left\{1, \ldots, m_{k}\right\}, \\
& L_{k} \leq q_{k, 0}+\sum_{t=1}^{k-1} q_{k, t} \cdot w_{t} \leq U_{k} .
\end{aligned}
$$


Although Problem $(A A R C)$ is still a semi-infinite LP (because of the requirement of robust constraint feasibility, $\forall \mathbf{w}$ ), since all the constraints are inequalities that are biaffine in the decision variables and the uncertain quantities, a very compact reformulation of the problem is available. In particular, with a typical constraint in $(A A R C)$ written as

$$
\lambda_{0}(\mathbf{x})+\sum_{t=1}^{T} \lambda_{t}(\mathbf{x}) \cdot w_{t} \leq 0, \quad \forall \mathbf{w} \in \mathscr{H}_{T+1},
$$

where $\lambda_{i}(\mathbf{x})$ are affine functions of the decision variables $\mathbf{x}$, it can be shown (see Ben-Tal and Nemirovski [3], Ben-Tal et al. [7] for details) that the previous condition is equivalent to

$$
\begin{gathered}
\lambda_{0}(\mathbf{x})+\sum_{t=1}^{T}\left(\lambda_{t}(\mathbf{x}) \cdot \frac{\underline{w}_{t}+\bar{w}_{t}}{2}+\frac{\bar{w}_{t}-\underline{w}_{t}}{2} \cdot \xi_{t}\right) \leq 0 \\
-\xi_{t} \leq \lambda_{t}(\mathbf{x}) \leq \xi_{t}, \quad t=1, \ldots, T,
\end{gathered}
$$

which are linear constraints in the decision variables $\mathbf{x}, \boldsymbol{\xi}$. Therefore, $(A A R C)$ can be reformulated as a linear program, with $O\left(T^{2} \cdot \max _{k} m_{k}\right)$ variables and $O\left(T^{2} \cdot \max _{k} m_{k}\right)$ constraints, which can be solved very efficiently using commercially available software.

We conclude our observations by making one last remark related to an immediate extension of the results. Note that in the statement of Problem 1, there was no mention about constraints on the states $x_{k}$ of the dynamical system. In particular, one may want to incorporate lower or upper bounds on the states as well,

$$
L_{k}^{x} \leq x_{k} \leq U_{k}^{x}
$$

We claim that, in case the mathematical problem, including such constraints, remains feasible, ${ }^{3}$ then affine policies are again optimal. The reason is that such constraints can always be simulated in our current framework, by adding suitable convex barriers to the stage costs $h_{k}\left(x_{k+1}\right)$. In particular, by considering the modified convex stage costs

$$
\tilde{h}_{k}\left(x_{k+1}\right) \stackrel{\text { def }}{=} h_{k}\left(x_{k+1}\right)+\mathbf{1}_{\left[L_{k+1}^{x}, U_{k+1}^{x}\right]}\left(x_{k+1}\right) \text {, }
$$

where $\mathbf{1}_{S}(x) \stackrel{\text { def }}{=}\{0$, if $x \in S ; \infty$, otherwise $\}$, it can be easily seen that the original problem, with convex stage costs $h_{k}(\cdot)$ and state constraints (13), is equivalent to a problem with the modified stage costs $\tilde{h}_{k}(\cdot)$ and no state constraints. And, since affine policies are optimal for the latter problem, the result is immediate. Therefore, our decision to exclude such constraints from the original formulation was made only for sake of brevity and conciseness of the proofs, but without loss of generality.

4. Proof of main theorem. The current section contains the proof of Theorem 3.1. Before presenting the details, we first give some intuition behind the strategy of the proof, and introduce the organization of the material.

Unlike most DP proofs, which use backward induction on the time periods, we proceed with a forward induction. Section 4.1 presents a test of the first step of the induction, and then introduces a detailed analysis of the consequences of the induction hypothesis.

We then separate the completion of the induction step into two parts. In the first part, discussed in $\S 4.2$, by exploiting the structure provided by the forward induction hypothesis, and making critical use of the properties of the optimal control law $u_{k}^{\star}\left(x_{k}\right)$ and optimal value function $J_{k}^{\star}\left(x_{k}\right)$ (the DP solutions), we introduce a candidate affine policy $q_{k}\left(\mathbf{w}_{k}\right)$. In $\$ 4.2 .1$, we then prove that this policy is robustly feasible, and preserves the minmax value of the overall problem, $J_{1}^{\star}\left(x_{1}\right)$, when used in conjunction with the original, convex state costs, $h_{k}\left(x_{k+1}\right)$.

Similarly, for the second part of the inductive step ( $\$ 4.3)$, by reanalyzing the feasible sets of the optimization problems resulting after the use of the (newly computed) affine policy $q_{k}\left(\mathbf{w}_{k}\right)$, we determine a candidate affine cost $z_{k}\left(\mathbf{w}_{k+1}\right)$, which we prove to be always larger than the original convex state costs, $h_{k}\left(x_{k+1}\right)$. However, despite this fact, in \$4.3.1, we also show that when this affine cost is incurred, the overall min-max value $J_{1}^{\star}\left(x_{1}\right)$ remains unchanged, which completes the proof of the inductive step.

Section 4.4 concludes the proof of Theorem 3.1, and outlines several counterexamples that prevent an immediate extension of the result to more general cases.

${ }^{3}$ Such constraints may lead to infeasible problems, for example, $T=1, x_{1}=0, u_{1} \in[0,1], w_{1} \in[0,1], x_{2} \in[5,10]$. 
4.1. Induction hypothesis. As mentioned before, the proof of the theorem uses a forward induction on the time step $k$. We begin by verifying the induction at $k=1$.

Using the same notation as in $\S 2$, by taking the affine control to be $q_{1} \stackrel{\text { def }}{=} u_{1}^{\star}\left(x_{1}\right)$, we immediately get that $q_{1}$, which is simply a constant, is robustly feasible, so (11a) is obeyed. Furthermore, since $u_{1}^{\star}\left(x_{1}\right)$ is optimal, we can write the overall optimal objective value as

$$
\begin{aligned}
J_{1}^{\star}\left(x_{1}\right) & =\min _{u_{1} \in\left[L_{1}, U_{1}\right]}\left[c_{1} \cdot u_{1}+g_{1}\left(x_{1}+u_{1}\right)\right]=c_{1} \cdot q_{1}+g_{1}\left(x_{1}+q_{1}\right)=\left(\text { by }(7 \mathrm{~b}) \text { and convexity of } h_{1}, J_{2}^{\star}\right) \\
& =c_{1} \cdot q_{1}+\max \left\{\left(h_{1}+J_{2}^{\star}\right)\left(x_{1}+q_{1}+\underline{w}_{1}\right),\left(h_{1}+J_{2}^{\star}\right)\left(x_{1}+q_{1}+\bar{w}_{1}\right)\right\} .
\end{aligned}
$$

Next, we introduce the affine cost $z_{1}\left(w_{1}\right) \stackrel{\text { def }}{=} z_{1,0}+z_{1,1} \cdot w_{1}$, where we constrain the coefficients $z_{1, i}$ to satisfy the following two linear equations:

$$
z_{1,0}+z_{1,1} \cdot w_{1}=h_{1}\left(x_{1}+q_{1}+w_{1}\right), \quad \forall w_{1} \in\left\{\underline{w}_{1}, \bar{w}_{1}\right\}
$$

Note that for fixed $x_{1}$ and $q_{1}$, the function $z_{1}\left(w_{1}\right)$ is nothing but a linear interpolation of the mapping $w_{1} \mapsto$ $h_{1}\left(x_{1}+q_{1}+w_{1}\right)$, matching the value at points $\left\{\underline{w}_{1}, \bar{w}_{1}\right\}$. Since $h_{1}$ is convex, the linear interpolation defined above clearly dominates it, so condition (11b) is readily satisfied. Furthermore, by (14), $J_{1}^{\star}\left(x_{1}\right)$ is achieved for $w_{1} \in\left\{\underline{w}_{1}, \bar{w}_{1}\right\}$, so condition (11c) is also obeyed.

Having checked the induction at time $k=1$, let us now assume that the statements of Theorem 3.1 are true for times $t=1, \ldots, k$. Equation (11c) written for stage $k$ then yields

$$
J_{1}^{\star}\left(x_{1}\right)=\max _{w_{k+1} \in \mathscr{P}_{k+1}}\left[\sum_{t=1}^{k}\left(c_{t} \cdot q_{t}\left(\mathbf{w}_{t}\right)+z_{t}\left(\mathbf{w}_{t+1}\right)\right)+J_{k+1}^{\star}\left(x_{1}+\sum_{t=1}^{k}\left(q_{t}\left(\mathbf{w}_{t}\right)+w_{t}\right)\right)\right]=\max _{\left(\theta_{1}, \theta_{2}\right) \in \Theta}\left[\theta_{1}+J_{k+1}^{\star}\left(\theta_{2}\right)\right],
$$

where

$$
\Theta \stackrel{\text { def }}{=}\left\{\left(\theta_{1}, \theta_{2}\right) \in \mathbb{R}^{2}: \theta_{1} \stackrel{\text { def }}{=} \sum_{t=1}^{k}\left(c_{t} \cdot q_{t}\left(\mathbf{w}_{t}\right)+z_{t}\left(\mathbf{w}_{t+1}\right)\right), \theta_{2} \stackrel{\text { def }}{=} x_{1}+\sum_{t=1}^{k}\left(q_{t}\left(\mathbf{w}_{t}\right)+w_{t}\right), \mathbf{w}_{k+1} \in \mathscr{H}_{k+1}\right\} .
$$

Since $\left\{q_{t}\right\}_{1 \leq t \leq k}$ and $\left\{z_{t}\right\}_{1 \leq t \leq k}$ are affine functions, this implies that, although the uncertainties $\mathbf{w}_{k+1}=$ $\left(w_{1}, \ldots, w_{k}\right)$ lie in a set with $2^{k}$ vertices (the hyperrectangle $\left.\mathscr{H}_{k+1}\right)$, they are only able to affect the objective through two affine combinations $\left(\theta_{1}\right.$ summarizing all the past stage costs, and $\theta_{2}$ representing the next state, $\left.x_{k+1}\right)$, taking values in the set $\Theta$. Such a polyhedron, arising as a two-dimensional affine projection of a $k$ dimensional hyperrectangle, is called a zonogon (see Figure 2 for an example). It belongs to a larger class of polytopes, known as zonotopes, whose combinatorial structure and properties are well documented in the discrete and computational geometry literature. The interested reader is referred to Chapter 7 of Ziegler [34] for a very nice and accessible introduction.

The main properties of a zonogon that we are interested in are summarized in Lemma 2 in the appendix. In particular, the set $\Theta$ is centrally symmetric, and has at most $2 k$ vertices (see Figure 2 for an example). Furthermore, by numbering the vertices of $\Theta$ in counterclockwise fashion, starting at

$$
\mathbf{v}_{0} \equiv \mathbf{v}_{\min } \stackrel{\text { def }}{=} \arg \max \left\{\theta_{1}: \boldsymbol{\theta} \in \arg \min \left\{\theta_{2}^{\prime}: \boldsymbol{\theta}^{\prime} \in \Theta\right\}\right\},
$$

we establish the following result concerning the points of $\Theta$ that are relevant in our problem.

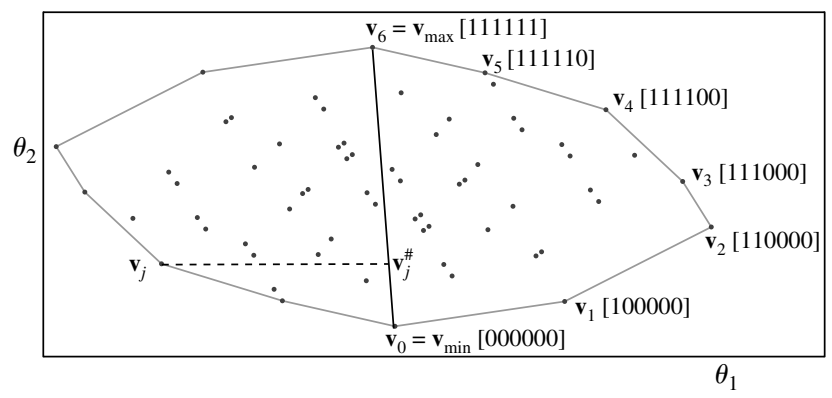

FIGURE 2. Zonogon obtained from projecting a hypercube in $\mathbb{R}^{6}$. 
Lemma 4.1. The maximum value in (15) is achieved for some $\left(\theta_{1}, \theta_{2}\right) \in\left\{\mathbf{v}_{0}, \mathbf{v}_{1}, \ldots, \mathbf{v}_{k}\right\}$.

Proof. The optimization problem described in (15) and (16) is a maximization of a convex function over a convex set. Therefore (see $\$ 32$ of Rockafellar [30]) the maximum is achieved at the extreme points of the set $\Theta$; namely, on the set $\left\{\mathbf{v}_{0}, \mathbf{v}_{1}, \ldots, \mathbf{v}_{2 p-1}, \mathbf{v}_{2 p} \equiv \mathbf{v}_{0}\right\}$, where $2 p$ is the number of vertices of $\Theta$. Letting $\mathbf{O}$ denote the center of $\Theta$, by part (iii) of Lemma .2 in the appendix, we have that the vertex symmetrically opposed to $\mathbf{v}_{\min }$; namely, $\mathbf{v}_{\max } \stackrel{\text { def }}{=} 2 \mathbf{O}-\mathbf{v}_{\min }$, satisfies $\mathbf{v}_{\max }=\mathbf{v}_{p}$.

Consider any vertex $\mathbf{v}_{j}$ with $j \in\{p+1, \ldots, 2 p-1\}$. From the definition of $\mathbf{v}_{\min }, \mathbf{v}_{\max }$, for any such vertex, there exists a point $\mathbf{v}_{j}^{\#} \in\left[\mathbf{v}_{\min }, \mathbf{v}_{\max }\right]$, with the same $\theta_{2}$-coordinate as $\mathbf{v}_{j}$, but with a $\theta_{1}$-coordinate larger than $\mathbf{v}_{j}$ (refer to Figure 2). Since such a point will have an objective in Problem (15) at least as large as $\mathbf{v}_{j}$, and $\mathbf{v}_{j}^{\#} \in\left[\mathbf{v}_{0}, \mathbf{v}_{p}\right]$, we can immediately conclude that the maximum of Problem (15) is achieved on the set $\left\{\mathbf{v}_{0}, \ldots, \mathbf{v}_{p}\right\}$. Since $2 p \leq 2 k$ (see part (ii) of Lemma .2), we immediately arrive at the conclusion of the lemma.

Since the argument presented in the lemma is recurring throughout several of our proofs and constructions, we end this subsection by introducing two useful definitions, and generalizing the previous result.

Consider the system of coordinates $\left(\theta_{1}, \theta_{2}\right)$ in $\mathbb{R}^{2}$, and let $\mathscr{S} \subset \mathbb{R}^{2}$ denote an arbitrary, finite set of points and $\mathscr{P}$ denote any (possibly nonconvex) polygon such that its set of vertices is exactly $\mathscr{S}$. With $\mathbf{y}_{\text {min }} \stackrel{\text { def }}{=} \arg \max \left\{\theta_{1}: \boldsymbol{\theta} \in\right.$ $\left.\arg \min \left\{\theta_{2}^{\prime}: \boldsymbol{\theta}^{\prime} \in \mathscr{P}\right\}\right\}$ and $\mathbf{y}_{\max } \stackrel{\text { def }}{=} \arg \max \left\{\theta_{1}: \boldsymbol{\theta} \in \arg \max \left\{\theta_{2}^{\prime}: \boldsymbol{\theta}^{\prime} \in \mathscr{P}\right\}\right\}$, by numbering the vertices of the convex hull of $\mathscr{S}$ in a counterclockwise fashion, starting at $\mathbf{y}_{0} \stackrel{\text { def }}{=} \mathbf{y}_{\min }$, and with $\mathbf{y}_{m}=\mathbf{y}_{\max }$, we define the right side of $\mathscr{P}$ and the zonogon hull of $\mathscr{S}$ as follows.

DEFINITION 4.1. The right side of an arbitrary polygon $\mathscr{P}$ is

$$
\text { r-side }(\mathscr{P}) \stackrel{\text { def }}{=}\left\{\mathbf{y}_{0}, \mathbf{y}_{1}, \ldots, \mathbf{y}_{m}\right\} .
$$

DeFINITION 4.2. The zonogon hull of a set of points $\mathscr{S}$ is

$$
\text { z-hull }(\mathscr{S}) \stackrel{\text { def }}{=}\left\{\mathbf{y} \in \mathbb{R}^{2}: \mathbf{y}=\mathbf{y}_{0}+\sum_{i=1}^{m} w_{i} \cdot\left(\mathbf{y}_{i}-\mathbf{y}_{i-1}\right), 0 \leq w_{i} \leq 1\right\} .
$$

Intuitively, r-side $(\mathscr{P})$ represents exactly what the names hints at, i.e., the vertices found on the right side of $\mathscr{P}$. An equivalent definition using more familiar operators could be

$$
\text { r-side }(\mathscr{P}) \equiv \operatorname{ext}\left(\operatorname{cone}\left(\left[\begin{array}{c}
-1 \\
0
\end{array}\right]\right)+\operatorname{conv}(\mathscr{P})\right)
$$

where $\operatorname{cone}(\cdot)$ and $\operatorname{conv}(\cdot)$ represent the conic and convex hull, respectively, and $\operatorname{ext}(\cdot)$ denotes the set of extreme points.

Using Definition .1 in $\S$ A2 of the appendix, one can see that the zonogon hull of a set $\mathscr{S}$ is simply a zonogon that has exactly the same vertices on the right side as the convex hull of $\mathscr{S}$, i.e., r-side $(\mathrm{z}$-hull $(\mathscr{S}))=$ r-side $(\operatorname{conv}(\mathscr{S})$ ). Some examples of zonogon hulls are shown in Figure 3 (note that the initial points in $\mathscr{S}$ do not necessarily fall inside the zonogon hull, and as such, there is no general inclusion relation between the zonogon hull and the convex hull). The reason for introducing this object is that it allows for the following immediate generalization of Lemma 4.1.

CoRollary 4.1. If $\mathscr{P}$ is any polygon in $\mathbb{R}^{2}$ (coordinates $\left.\left(\theta_{1}, \theta_{2}\right) \equiv \boldsymbol{\theta}\right)$ with a finite set $\mathscr{S}$ of vertices, and $f(\boldsymbol{\theta}) \stackrel{\text { def }}{=} \theta_{1}+g\left(\theta_{2}\right)$, where $g: \mathbb{R} \rightarrow \overline{\mathbb{R}}$ is any convex function, then the following chain of equalities holds:

$$
\max _{\boldsymbol{\theta} \in \mathscr{P}} f(\boldsymbol{\theta})=\max _{\theta \in \operatorname{conv}(\mathscr{P})} f(\boldsymbol{\theta})=\max _{\theta \in \mathscr{S}} f(\boldsymbol{\theta})=\max _{\theta \in \mathrm{r} \text {-side }(\mathscr{P})} f(\boldsymbol{\theta})=\max _{\theta \in z \text {-hull }(\mathscr{S})} f(\boldsymbol{\theta})=\max _{\theta \in \operatorname{r-side}(\mathrm{z} \text {-hull }(\mathscr{S}))} f(\boldsymbol{\theta}) .
$$
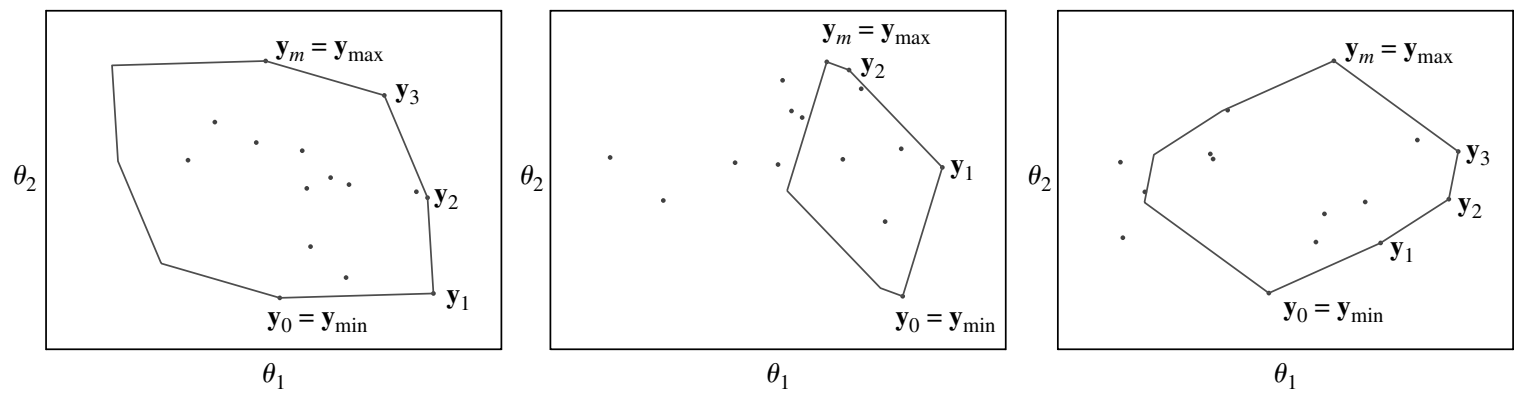

FIGURE 3. Examples of zonogon hulls for different sets $\mathscr{S} \in \mathbb{R}^{2}$. 
Proof. The proof is identical to that of Lemma 4.1, and is omitted for brevity.

Using this result, whenever we are faced with a maximization of a convex function $\theta_{1}+g\left(\theta_{2}\right)$, we can switch between different feasible sets, without affecting the overall optimal value of the optimization problem.

In the context of Lemma 4.1, the above result allows us to restrict attention from a potentially large set of relevant points (the $2^{k}$ vertices of the hyperrectangle $\mathscr{H}_{k+1}$ ), to the $k+1$ vertices found on the right side of the zonogon $\Theta$, which also gives insight into why the construction of an affine controller $q_{k+1}\left(\mathbf{w}_{k+1}\right)$ with $k+1$ degrees of freedom, yielding the same overall objective function value $J_{m M}$, might actually be possible.

In the remaining part of $\$ 4.1$, we further narrow down this set of relevant points, by using the structure and properties of the optimal control law $u_{k+1}^{\star}\left(x_{k+1}\right)$ and optimal value function $J_{k+1}^{\star}\left(x_{k+1}\right)$, derived in $\S 2$. Before proceeding, however, we first reduce the notational clutter by introducing several simplifications and assumptions.

4.1.1. Simplified notation and assumptions. To start, we omit the time subscript $k+1$ whenever possible, so that we write $\mathbf{w}_{k+1} \equiv \mathbf{w}, q_{k+1}(\cdot) \equiv q(\cdot), J_{k+1}^{\star}(\cdot) \equiv J^{\star}(\cdot), g_{k+1}(\cdot) \equiv g(\cdot)$. The affine functions $\theta_{1,2}\left(\mathbf{w}_{k+1}\right)$ and $q_{k+1}\left(\mathbf{w}_{k+1}\right)$ are identified as

$$
\theta_{1}(\mathbf{w}) \stackrel{\text { def }}{=} a_{0}+\mathbf{a}^{\prime} \mathbf{w} ; \quad \theta_{2}(\mathbf{w}) \stackrel{\text { def }}{=} b_{0}+\mathbf{b}^{\prime} \mathbf{w} ; \quad q(\mathbf{w}) \stackrel{\text { def }}{=} q_{0}+\mathbf{q}^{\prime} \mathbf{w},
$$

where $\mathbf{a}, \mathbf{b} \in \mathbb{R}^{k}$ are the generators of the zonogon $\Theta$. Since $\theta_{2}$ is nothing but the state $x_{k+1}$, instead of referring to $J_{k+1}^{\star}\left(x_{k+1}\right)$ and $u_{k+1}^{\star}\left(x_{k+1}\right)$, we use $J^{\star}\left(\theta_{2}\right)$ and $u^{\star}\left(\theta_{2}\right)$.

Since our exposition relies heavily on sets given by maps $\gamma: \mathbb{R}^{k} \mapsto \mathbb{R}^{2}(k \geq 2)$, to reduce the number of symbols, we denote the resulting coordinates in $\mathbb{R}^{2}$ by $\gamma_{1}, \gamma_{2}$ and use the following overloaded notation:

- $\gamma_{i}[\mathbf{v}]$ denotes the $\gamma_{i}$-coordinate of the point $\mathbf{v} \in \mathbb{R}^{2}$,

- $\gamma_{i}(\mathbf{w})$ is the value assigned by the $i$-th component of the map $\boldsymbol{\gamma}$ to $\mathbf{w} \in \mathbb{R}^{k}$ (equivalently, $\gamma_{i}(\mathbf{w}) \equiv \gamma_{i}[\boldsymbol{\gamma}(\mathbf{w})]$ ). The different use of parentheses should remove any ambiguity from the notation (particularly in the case $k=2$ ). For the same $\left(\gamma_{1}, \gamma_{2}\right)$ coordinate system, we use $\operatorname{cotan}(\mathbf{M}, \mathbf{N})$ to denote the cotangent of the angle formed by an oriented line segment $[\mathbf{M}, \mathbf{N}] \in \mathbb{R}^{2}$ with the $\gamma_{1}$-axis,

$$
\operatorname{cotan}(\mathbf{M}, \mathbf{N}) \stackrel{\text { def }}{=} \frac{\gamma_{1}[\mathbf{N}]-\gamma_{1}[\mathbf{M}]}{\gamma_{2}[\mathbf{N}]-\gamma_{2}[\mathbf{M}]} .
$$

Also, to avoid writing multiple functional compositions, since most quantities of interest depend solely on the state $x_{k+1}$ (which is the same as $\theta_{2}$ ), we use the following shorthand notation for any point $\mathbf{v} \in \mathbb{R}^{2}$, with corresponding $\theta_{2}$-coordinate given by $\theta_{2}[\mathbf{v}]$ :

$$
u^{\star}\left(\theta_{2}[\mathbf{v}]\right) \equiv u^{\star}(\mathbf{v}) ; \quad J^{\star}\left(\theta_{2}[\mathbf{v}]\right) \equiv J^{\star}(\mathbf{v}) ; \quad g\left(\theta_{2}[\mathbf{v}]+u^{\star}\left(\theta_{2}[\mathbf{v}]\right)\right) \equiv g(\mathbf{v}) .
$$

We use the same counterclockwise numbering of the vertices of $\Theta$ as introduced earlier in $\S 4.1$ :

$$
\mathbf{v}_{0} \stackrel{\text { def }}{=} \mathbf{v}_{\text {min }}, \ldots, \mathbf{v}_{p} \stackrel{\text { def }}{=} \mathbf{v}_{\text {max }}, \ldots, \mathbf{v}_{2 p}=\mathbf{v}_{\text {min }}
$$

where $2 p$ is the number of vertices of $\Theta$, and we also make the following simplifying assumptions.

Assumption 4.1. The uncertainty vector at time $k+1, \mathbf{w}_{k+1}=\left(w_{1}, \ldots, w_{k}\right)$, belongs to the unit hypercube of $\mathbb{R}^{k}$, i.e., $\mathscr{H}_{k+1}=\mathscr{W}_{1} \times \cdots \times \mathscr{W}_{k} \equiv[0,1]^{k}$.

Assumption 4.2. The zonogon $\Theta$ has a maximal number of vertices, i.e., $p=k$.

Assumption 4.3. The vertex of the hypercube projecting to $\mathbf{v}_{i}, i \in\{0, \ldots, k\}$, is exactly $[1,1, \ldots, 1$, $0, \ldots, 0]$, i.e., 1 in the first $i$ components and 0 thereafter (see Figure 2).

These assumptions are made only to facilitate the exposition, and result in no loss of generality. To see this, note that the conditions of Assumption 4.1 can always be achieved by adequate translation and scaling of the generators $\mathbf{a}$ and $\mathbf{b}$ (refer to $\S \mathrm{A} 2$ in the appendix for more details), and Assumption 4.3 can be satisfied by renumbering and possibly reflecting ${ }^{4}$ the coordinates of the hyperrectangle, i.e., the disturbances $w_{1}, \ldots, w_{k}$. As for Assumption 4.2, we argue that an extension of our construction to the degenerate case $p<k$ is immediate (one could also remove the degeneracy by applying an infinitesimal perturbation to the generators $\mathbf{a}$ or $\mathbf{b}$, with infinitesimal cost implications).

${ }^{4}$ Reflection would represent a transformation $w_{i} \mapsto 1-w_{i}$. As we show in a later result (Lemma 4.4 of $\$ 4.2 .1$ ), reflection is actually not needed, but this is not obvious at this point. 
4.1.2. Further analysis of the induction hypothesis. In the simplified notation, Equation (15) can now be rewritten, using (9) to express $J^{\star}(\cdot)$ as a function of $u^{\star}(\cdot)$ and $g(\cdot)$, as follows:

$$
\begin{aligned}
(\text { OPT }) \quad J_{m M} & =\max _{\left(\gamma_{1}, \gamma_{2}\right) \in \Gamma^{\star}}\left[\gamma_{1}+g\left(\gamma_{2}\right)\right], \\
& \Gamma^{\star} \stackrel{\text { def }}{=}\left\{\left(\gamma_{1}^{\star}, \gamma_{2}^{\star}\right): \gamma_{1}^{\star} \stackrel{\text { def }}{=} \theta_{1}+c \cdot u^{\star}\left(\theta_{2}\right), \gamma_{2}^{\star} \stackrel{\text { def }}{=} \theta_{2}+u^{\star}\left(\theta_{2}\right),\left(\theta_{1}, \theta_{2}\right) \in \Theta\right\} .
\end{aligned}
$$

In this form, $(O P T)$ represents the optimization problem solved by the uncertainties $\mathbf{w} \in \mathscr{H}$ when the optimal policy, $u^{\star}(\cdot)$, is used at time $k+1$. The significance of $\gamma_{1,2}^{\star}$ in the context of the original problem is straightforward: $\gamma_{1}^{\star}$ stands for the cumulative past stage costs, plus the current stage control cost $c \cdot u^{\star}$, while $\gamma_{2}^{\star}$, which is the same variable as $y_{k+1}$, is the sum of the state and the control (in the inventory Example 1.1, it would represent the inventory position just after ordering, before seeing the demand).

Note that we have $\Gamma^{\star} \equiv \boldsymbol{\gamma}^{\star}(\Theta)$, where a characterization for the map $\boldsymbol{\gamma}^{\star}$ can be obtained by replacing the optimal policy, given by (8) in Equation (23b):

$$
\boldsymbol{\gamma}^{\star}: \mathbb{R}^{2} \rightarrow \mathbb{R}^{2}, \boldsymbol{\gamma}^{\star}(\boldsymbol{\theta}) \equiv\left(\gamma_{1}^{\star}(\boldsymbol{\theta}), \gamma_{2}^{\star}(\boldsymbol{\theta})\right)= \begin{cases}\left(\theta_{1}+c \cdot U, \theta_{2}+U\right) & \text { if } \theta_{2}<y^{\star}-U, \\ \left(\theta_{1}-c \cdot \theta_{2}+c \cdot y^{\star}, y^{\star}\right) & \text { otherwise, } \\ \left(\theta_{1}+c \cdot L, \theta_{2}+L\right) & \text { if } \theta_{2}>y^{\star}-L .\end{cases}
$$

The following is a compact characterization for the maximizers in problem (OPT) from (23a).

Lemma 4.2. The maximum in problem $(O P T)$ over $\Gamma^{\star}$ is reached on the right side of

$$
\Delta_{\Gamma^{\star}} \stackrel{\text { def }}{=} \operatorname{conv}\left(\left\{\mathbf{y}_{0}^{\star}, \ldots, \mathbf{y}_{k}^{\star}\right\}\right)
$$

where

$$
\mathbf{y}_{i}^{\star} \stackrel{\text { def }}{=} \boldsymbol{\gamma}^{\star}\left(\mathbf{v}_{i}\right)=\left(\theta_{1}\left[\mathbf{v}_{i}\right]+c \cdot u^{\star}\left(\mathbf{v}_{i}\right), \theta_{2}\left[\mathbf{v}_{i}\right]+u^{\star}\left(\mathbf{v}_{i}\right)\right), \quad i \in\{0, \ldots, k\} .
$$

Proof. By Lemma 4.1, the maximum in (15) is reached at one of the vertices $\mathbf{v}_{0}, \mathbf{v}_{1}, \ldots, \mathbf{v}_{k}$ of the zonogon $\Theta$. Because this problem is equivalent to problem $(O P T)$ in $(23 \mathrm{a})$, written over $\Gamma^{\star}$, we can immediately conclude that the maximum of the latter problem is reached at the points $\left\{\mathbf{y}_{i}^{\star}\right\}_{1 \leq i \leq k}$ given by (26). Furthermore, since $g(\cdot)$ is convex (see Property (P2) of the optimal DP solution in $\$ 2$ ), we can apply Corollary 4.1, and replace the points $\mathbf{y}_{i}^{\star}$ with the right side of their convex hull, r-side $\left(\Delta_{\Gamma^{\star}}\right)$, without changing the result of the optimization problem, which completes the proof.

Because this result is central to our future construction and proof, we spend the remaining part of the subsection discussing some of the properties of the main object of interest, the set r-side $\left(\Delta_{\Gamma^{*}}\right)$. To understand the geometry of the set $\Delta_{\Gamma^{\star}}$ and its connection with the optimal control law, note that the mapping $\gamma^{\star}$ from $\Theta$ to $\Gamma^{\star}$ discriminates points $\boldsymbol{\theta}=\left(\theta_{1}, \theta_{2}\right) \in \Theta$, depending on their position relative to the horizontal band

$$
\mathscr{B}_{L U} \stackrel{\text { def }}{=}\left\{\left(\theta_{1}, \theta_{2}\right) \in \mathbb{R}^{2}: \theta_{2} \in\left[y^{\star}-U, y^{\star}-L\right]\right\} .
$$

In terms of the original problem, the band $\mathscr{B}_{L U}$ represents the portion of the state space $x_{k+1}$ (i.e., $\theta_{2}$ ) in which the optimal control policy $u^{\star}$ is unconstrained by the bounds $L, U$. More precisely, points below $\mathscr{B}_{L U}$ and points above $\mathscr{B}_{L U}$ correspond to state-space regions where the upper bound, $U$, and the lower bound, $L$, are active, respectively.

With respect to the geometry of $\Gamma^{\star}$, we can use (24) and the definition of $\mathbf{v}_{0}, \ldots, \mathbf{v}_{k}$ to distinguish a total of four distinct cases. The first three, shown in Figure 4, are very easy to analyze:

[C1] If the entire zonogon $\Theta$ falls below the band $\mathscr{B}_{L U}$, i.e., $\theta_{2}\left[\mathbf{v}_{k}\right]<y^{\star}-U$, then $\Gamma^{\star}$ is simply a translation of $\Theta$, by $(c \cdot U, U)$, so that r-side $\left(\Delta_{\Gamma^{\star}}\right)=\left\{\mathbf{y}_{0}^{\star}, \mathbf{y}_{1}^{\star}, \ldots, \mathbf{y}_{k}^{\star}\right\}$.

[C2] If $\Theta$ lies inside the band $\mathscr{B}_{L U}$, i.e., $y^{\star}-U \leq \theta_{2}\left[\mathbf{v}_{0}\right] \leq \theta_{2}\left[\mathbf{v}_{k}\right] \leq y^{\star}-L$, then all the points in $\Gamma^{\star}$ will have $\gamma_{2}^{\star}=y^{\star}$, so $\Gamma^{\star}$ will be a line segment, and $\left|\mathrm{r}-\operatorname{side}\left(\Delta_{\Gamma^{\star}}\right)\right|=1$. 

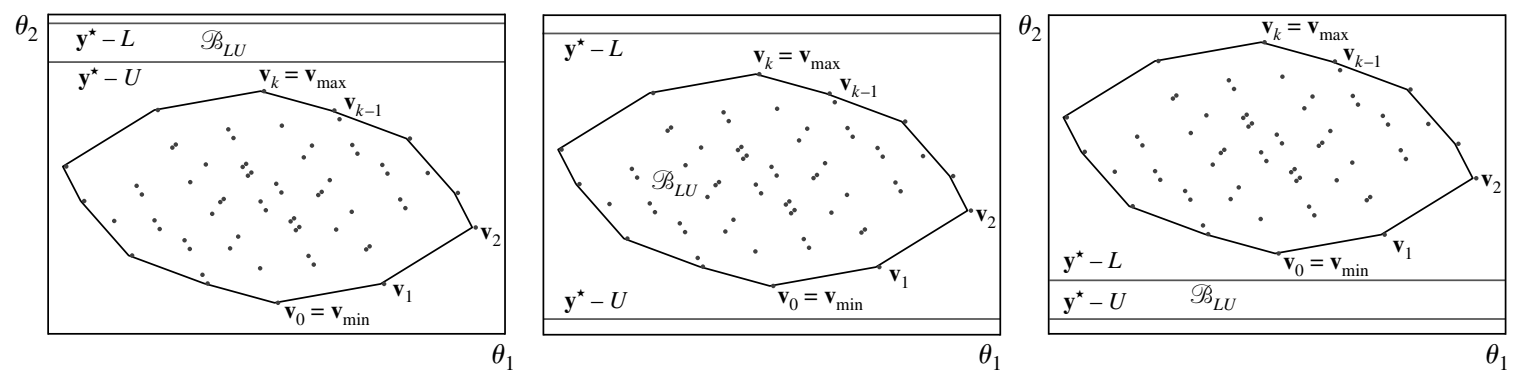

Figure 4. Trivial cases when zonogon $\Theta$ lies entirely [C1] below, [C2] inside, or [C3] above the band $\mathscr{\Re}_{L U}$.

[C3] If the entire zonogon $\Theta$ falls above the band $\mathscr{B}_{L U}$, i.e., $\theta_{2}\left[\mathbf{v}_{0}\right]>y^{\star}-L$, then $\gamma^{\star}$ is again a translation of $\Theta$, by $(c \cdot L, L)$, so, again r-side $\left(\Delta_{\Gamma^{\star}}\right)=\left\{\mathbf{y}_{0}^{\star}, \mathbf{y}_{1}^{\star}, \ldots, \mathbf{y}_{k}^{\star}\right\}$.

The remaining case, [C4], is when $\Theta$ intersects the horizontal band $\mathscr{B}_{L U}$ in a nontrivial fashion. We can separate this situation in the three subcases shown in Figure 5, depending on the position of the vertex $\mathbf{v}_{t} \in$ r-side $(\Theta)$, where the index $t$ relates the per unit control cost, $c$, with the geometrical properties of the zonogon:

$$
t \stackrel{\text { def }}{=} \begin{cases}0 & \text { if } \frac{a_{1}}{b_{1}} \leq c, \\ \max \left\{i \in\{1, \ldots, k\}: \frac{a_{i}}{b_{i}}>c\right\} & \text { otherwise. }\end{cases}
$$

We remark that the definition of $t$ is consistent, because by the simplifying Assumption 4.3, the generators $\mathbf{a}, \mathbf{b}$ of the zonogon $\Theta$ always satisfy

$$
\left\{\begin{array}{l}
\frac{a_{1}}{b_{1}}>\frac{a_{2}}{b_{2}}>\cdots>\frac{a_{k}}{b_{k}}, \\
b_{1}, b_{2}, \ldots, b_{k} \geq 0 .
\end{array}\right.
$$

An equivalent characterization of $\mathbf{v}_{t}$ can be obtained as the result of an optimization problem

$$
\mathbf{v}_{t} \equiv \arg \min \left\{\theta_{2}: \boldsymbol{\theta} \in \arg \max \left\{\theta_{1}^{\prime}-c \cdot \theta_{2}^{\prime}: \boldsymbol{\theta}^{\prime} \in \Theta\right\}\right\} .
$$

The following lemma summarizes all the relevant geometrical properties corresponding to this case.
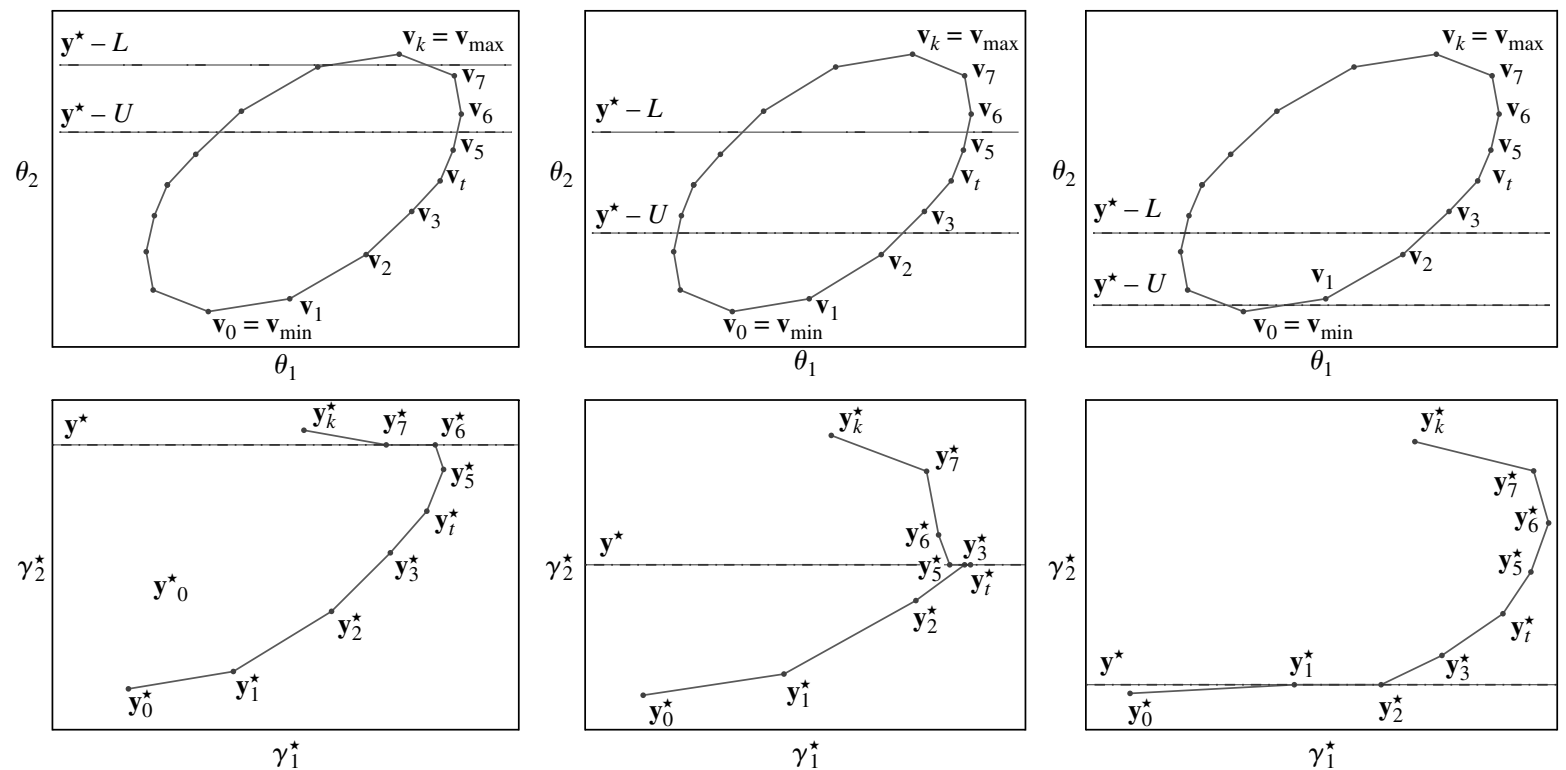

Figure 5. Case [C4]. Original zonogon $\Theta$ (first row) and the set $\Gamma^{\star}$ (second row) when $\mathbf{v}_{t}$ falls (a) under, (b) inside, or (c) above the band $\mathscr{B}_{L U}$. 
LeMma 4.3. When the zonogon $\Theta$ has a nontrivial intersection with the band $\mathscr{B}_{L U}$ (case [C4]), the convex polygon $\Delta_{\Gamma^{\star}}$ and the set of points on its right side, $\mathrm{r}$-side $\left(\Delta_{\Gamma^{\star}}\right)$, verify the following properties.

(i) $r$-side $\left(\Delta_{\Gamma^{\star}}\right)$ is the union of two sequences of consecutive vertices (one starting at $\mathbf{y}_{0}^{\star}$, and one ending at $\left.\mathbf{y}_{k}^{\star}\right)$, and possibly an additional vertex, $\mathbf{y}_{t}^{\star}$ :

$$
\mathrm{r} \text {-side }\left(\Delta_{\Gamma^{\star}}\right)=\left\{\mathbf{y}_{0}^{\star}, \mathbf{y}_{1}^{\star}, \ldots, \mathbf{y}_{s}^{\star}\right\} \cup\left\{\mathbf{y}_{t}^{\star}\right\} \cup\left\{\mathbf{y}_{r}^{\star}, \mathbf{y}_{r+1}^{\star} \ldots, \mathbf{y}_{k}^{\star}\right\}, \quad \text { for some } s \leq r \in\{0, \ldots, k\} .
$$

(ii) With $\operatorname{cotan}(\cdot, \cdot)$ given by (21) applied to the $\left(\gamma_{1}^{\star}, \gamma_{2}^{\star}\right)$ coordinates, we have that

$$
\left\{\begin{array}{l}
\operatorname{cotan}\left(\mathbf{y}_{s}^{\star}, \mathbf{y}_{\min (t, r)}^{\star}\right) \geq \frac{a_{s+1}}{b_{s+1}}, \\
\operatorname{cotan}\left(\mathbf{y}_{\max (t, s)}^{\star}, \mathbf{y}_{r}^{\star}\right) \leq \frac{a_{r}}{b_{r}}, \quad \text { whenever } t>s,
\end{array}\right.
$$

While the proof of the lemma is slightly technical (which is why we have decided to leave it for $\S A 3$ in the appendix), its implications are more straightforward. In conjuction with Lemma 4.2, it provides a compact characterization of the points $\mathbf{y}_{i}^{\star} \in \Gamma^{\star}$, which are potential maximizers of problem $(O P T)$ in (23a), which immediately narrows the set of relevant points $\mathbf{v}_{i} \in \Theta$ in optimization problem (15), and implicitly, the set of disturbances $\mathbf{w} \in \mathscr{H}_{k+1}$ that can achieve the overall min-max cost.

4.2. Construction of the affine control law. Having analyzed the consequences that result from using the induction hypothesis of Theorem 3.1, we now return to the task of completing the inductive proof, which amounts to constructing an affine control law $q_{k+1}\left(\mathbf{w}_{k+1}\right)$ and an affine cost $z_{k+1}\left(\mathbf{w}_{k+2}\right)$ that verify conditions (11a), (11b), and (11c) in Theorem 3.1. We separate this task into two parts. In the current section, we exhibit an affine control law $q_{k+1}\left(\mathbf{w}_{k+1}\right)$ that is robustly feasible, i.e., satisfies constraint (11a), and that leaves the overall min-max cost $J_{1}^{\star}\left(x_{1}\right)$ unchanged, when used at time $k+1$ in conjunction with the original convex state cost, $h_{k+1}\left(x_{k+2}\right)$. The second part of the induction, i.e., the construction of the affine costs $z_{k+1}\left(\mathbf{w}_{k+2}\right)$, is left for $\S 4.3$.

In the simplified notation introduced earlier, the problem we would like to solve is to find an affine control law $q(\mathbf{w})$ such that

$$
\begin{aligned}
J_{1}^{\star}\left(x_{1}\right) & =\max _{w \in \mathscr{H}_{k+1}}\left[\theta_{1}(\mathbf{w})+c \cdot q(\mathbf{w})+g\left(\theta_{2}(\mathbf{w})+q(\mathbf{w})\right)\right], \\
L & \leq q(\mathbf{w}) \leq U, \quad \forall \mathbf{w} \in \mathscr{H}_{k+1} .
\end{aligned}
$$

The maximization represents the problem solved by the disturbances, when the affine controller, $q(\mathbf{w})$, is used instead of the optimal controller, $u^{\star}\left(\theta_{2}\right)$. As such, the first equation amounts to ensuring that the overall objective function remains unchanged, and the inequalities are a restatement of the robust feasibility condition. The system can be immediately rewritten as

$$
\begin{aligned}
(\text { AFF }) \quad J_{1}^{\star}\left(x_{1}\right) & =\max _{\left(\gamma_{1}, \gamma_{2}\right) \in \Gamma}\left[\gamma_{1}+g\left(\gamma_{2}\right)\right] \\
L & \leq q(\mathbf{w}) \leq U, \quad \forall \mathbf{w} \in \mathscr{H}_{k+1},
\end{aligned}
$$

where

$$
\Gamma \stackrel{\text { def }}{=}\left\{\left(\gamma_{1}, \gamma_{2}\right): \gamma_{1} \stackrel{\text { def }}{=} \theta_{1}(\mathbf{w})+c \cdot q(\mathbf{w}), \gamma_{2} \stackrel{\text { def }}{=} \theta_{2}(\mathbf{w})+q(\mathbf{w}), \quad \mathbf{w} \in \mathscr{H}_{k+1}\right\} .
$$

With this reformulation, all our decision variables, i.e., the affine coefficients of $q(\mathbf{w})$, have been moved to the feasible set $\Gamma$ of the maximization problem $(A F F)$ in (31a). Note that with an affine controller $q(\mathbf{w})=q_{0}+\mathbf{q}^{\prime} \mathbf{w}$, and $\theta_{1,2}$ affine in $\mathbf{w}$, the feasible set $\Gamma$ will represent a new zonogon in $\mathbb{R}^{2}$, with generators given by $\mathbf{a}+c \cdot \mathbf{q}$ and $\mathbf{b}+\mathbf{q}$. Furthermore, since the function $g$ is convex, the optimization problem $(A F F)$ over $\Gamma$ is of the exact same nature as that in (15), defined over the zonogon $\Theta$. Thus, in perfect analogy with our discussion in $\S 4.1$ (Lemma 4.1 and Corollary 4.1), we can conclude that the maximum in $(A F F)$ must occur at a vertex of $\Gamma$ found in $\mathrm{r}$-side $(\Gamma)$.

In a different sense, note that optimization problem $(A F F)$ is also very similar to problem $(O P T)$ in (23b), which was the problem solved by the uncertainties $\mathbf{w}$ when the optimal control law $u^{\star}\left(\theta_{2}\right)$ was used at time $k+1$. Since the optimal value of the latter problem is exactly equal to the overall min-max value, $J_{1}^{\star}\left(x_{1}\right)$, we interpret the equation in (31a) as comparing the optimal values in the two optimization problems, (AFF) and $(O P T)$.

As such, note that the same convex objective function $\gamma_{1}+g\left(\gamma_{2}\right)$ is maximized in both problems, but over different feasible sets, $\Gamma^{\star}$ for $(O P T)$ and $\Gamma$ for $(A F F)$, respectively. From Lemma 4.2 in $\S 4.1 .2$, the maximum of problem $(O P T)$ is reached on the set r-side $\left(\Delta_{\Gamma^{\star}}\right)$, where $\Delta_{\Gamma^{\star}}=\operatorname{conv}\left(\left\{\mathbf{y}_{0}^{\star}, \mathbf{y}_{1}^{\star}, \ldots, \mathbf{y}_{k}^{\star}\right\}\right)$. From the discussion 
in the previous paragraph, the maximum in problem $(A F F)$ occurs on r-side $(\Gamma)$. Therefore, to compare the two results of the maximization problems, we must relate the sets r-side $\left(\Delta_{\Gamma^{*}}\right)$ and $\mathrm{r}$-side $(\Gamma)$.

In this context, we introduce the central idea behind the construction of the affine control law, $q(\mathbf{w})$. Recalling the concept of a zonogon hull introduced in Definition 4.2, we argue that, if the affine coefficients of the controller, $q_{0}$, $\mathbf{q}$, were computed in such a way that the zonogon $\Gamma$ actually corresponded to the zonogon hull of the set $\left\{\mathbf{y}_{0}^{\star}, \mathbf{y}_{1}^{\star}, \ldots, \mathbf{y}_{k}^{\star}\right\}$, then, by using the result in Corollary 4.1, we could immediately conclude that the optimal values in $(O P T)$ and $(A F F)$ are the same.

To this end, we introduce the following procedure for computing the affine control law $q(\mathbf{w})$.

Algorithm 1 (Compute affine controller $q(\mathbf{w})$ )

Require: $\theta_{1}(\mathbf{w}), \theta_{2}(\mathbf{w}), g(\cdot), u^{\star}(\cdot)$

1. if $\left(\Theta\right.$ falls below $\left.\mathscr{B}_{L U}\right)$ or $\left(\Theta \subseteq \mathscr{B}_{L U}\right)$ or $\left(\Theta\right.$ falls above $\left.\mathscr{B}_{L U}\right)$, then

2. Return $q(\mathbf{w})=u^{\star}\left(\theta_{2}(\mathbf{w})\right)$.

3. else

4. Apply the mapping (24) to obtain the points $\mathbf{y}_{i}^{\star}, i \in\{0, \ldots, k\}$.

5. Compute the set $\Delta_{\Gamma^{\star}}=\operatorname{conv}\left(\left\{\mathbf{y}_{0}^{\star}, \ldots, \mathbf{y}_{k}^{\star}\right\}\right)$.

6. $\quad$ Let r-side $\left(\Delta_{\Gamma^{\star}}\right)=\left\{\mathbf{y}_{0}^{\star}, \mathbf{y}_{1}^{\star}, \ldots, \mathbf{y}_{s}^{\star}\right\} \cup\left\{\mathbf{y}_{t}^{\star}\right\} \cup\left\{\mathbf{y}_{r}^{\star}, \ldots, \mathbf{y}_{k}^{\star}\right\}$.

7. Solve the following system for $q_{0}, \ldots, q_{k}$ and $K_{U}, K_{L}$ :

$$
\begin{cases}q_{0}+\cdots+q_{i}=u^{\star}\left(\mathbf{v}_{i}\right), & \forall \mathbf{y}_{i}^{\star} \in \mathrm{r}-\operatorname{side}\left(\Delta_{\Gamma^{\star}}\right) \quad(\text { matching }), \\ \frac{a_{i}+c \cdot q_{i}}{b_{i}+q_{i}}=K_{U}, & \forall i \in\{s+1, \ldots, \min (t, r)\} \quad \text { (alignment below } t), \\ \frac{a_{i}+c \cdot q_{i}}{b_{i}+q_{i}}=K_{L}, & \forall i \in\{\max (t, s)+1, \ldots, r\} \quad \text { (alignment above } t) .\end{cases}
$$

8. Return $q(\mathbf{w})=q_{0}+\sum_{i=1}^{k} q_{i} w_{i}$.

9. end if

Before proving that the construction is well defined and produces the expected result, we first give some intuition for the constraints in system (33). To have the zonogon $\Gamma$ be the same as the zonogon hull of $\left\{\mathbf{y}_{0}^{\star}, \ldots, \mathbf{y}_{k}^{\star}\right\}$, we must ensure that the vertices on the right side of $\Gamma$ exactly correspond to the points on the right side of $\Delta_{\Gamma^{\star}}=\operatorname{conv}\left(\left\{\mathbf{y}_{0}^{\star}, \ldots, \mathbf{y}_{k}^{\star}\right\}\right)$. This is achieved in two stages. First, we ensure that vertices $\mathbf{w}_{i}$ of the hypercube $\mathscr{H}_{k+1}$ that are mapped by the optimal control law $u^{\star}(\cdot)$ into points $\mathbf{v}_{i}^{\star} \in \mathrm{r}$-side $\left(\Delta_{\Gamma^{\star}}\right)$ (through the succession of mappings $\mathbf{w}_{i} \stackrel{(16)}{\mapsto} \mathbf{v}_{i} \in \mathrm{r}$-side $(\Theta) \stackrel{(26)}{\mapsto} \mathbf{y}_{i}^{\star} \in \mathrm{r}$-side $\left.\left(\Delta_{\Gamma^{*}}\right)\right)$, will be mapped by the affine control law, $q\left(\mathbf{w}_{i}\right)$, into the same point $\mathbf{y}_{i}^{\star}$ (through the mappings $\mathbf{w}_{i} \stackrel{(16)}{\mapsto} \mathbf{v}_{i} \in \mathrm{r}$-side $(\Theta) \stackrel{(32)}{\mapsto} \mathbf{y}_{i}^{\star} \in \mathrm{r}$-side $\left(\Delta_{\Gamma^{\star}}\right)$ ). This is done in the first set of constraints, by matching the value of the optimal control law at any such points. Second, we ensure that any such matched points $\mathbf{y}_{i}^{\star}$ actually correspond to the vertices on the right side of the zonogon $\Gamma$. This is done in the second and third set of constraints in (33), by computing the affine coefficients $q_{j}$ in such a way that the resulting segments in the generators of the zonogon $\Gamma$, namely $\left(\begin{array}{c}a_{j}+c \cdot q_{j} \\ b_{j}+q_{j}\end{array}\right)$, are all aligned, i.e., have the same cotangent, given by the $K_{U}, K_{L}$ variables. Geometrically, this exactly corresponds to the situation shown in Figure 6.

We remark that the above algorithm does not explicitly require that the control $q(\mathbf{w})$ be robustly feasible, i.e., condition (31b). However, this condition turns out to hold as a direct result of the way matching and alignment are performed in Algorithm 1.

4.2.1. Affine controller preserves overall objective and is robust. In this section, we prove that the affine control law $q(\mathbf{w})$ produced by Algorithm 1 satisfies the requirements of (31a), i.e., it is robustly feasible, and it preserves the overall objective function $J_{1}^{\star}\left(x_{1}\right)$, when used in conjunction with the original convex state costs, $h(\cdot)$. With the exception of Corollary 4.1, all the key results that we are using are contained in $\S 4.1 .2$ (Lemmas 4.2 and 4.3). Therefore, we preserve the same notation and case discussion as initially introduced there.

First, consider the condition on line 1 of Algorithm 1, and note that this corresponds to the three trivial cases [C1], [C2], and [C3] of §4.1.2. In particular, since $\theta_{2} \equiv x_{k+1}$, we can use (8) to conclude that in these cases, the optimal control law $u^{\star}(\cdot)$ is actually affine:

[C1] If $\Theta$ falls below the band $\mathscr{B}_{L U}$, then the upper bound constraint on the control at time $k$ is always active, i.e., $u^{\star}\left(\theta_{2}(\mathbf{w})\right)=U, \forall \mathbf{w} \in \mathscr{H}_{k+1}$. 



FiguRE 6. Outcomes from the matching and alignment performed in Algorithm 1.

[C2] If $\Theta \subseteq \mathscr{P}_{L U}$, then the constraints on the control at time $k$ are never active, i.e., $u^{\star}\left(\theta_{2}(\mathbf{w})\right)=y^{\star}-\theta_{2}(\mathbf{w})$, hence affine in $\mathbf{w}$, since $\theta_{2}$ is affine in $\mathbf{w}$, by (20).

[C3] If $\Theta$ falls above the band $\mathscr{B}_{L U}$, then the lower-bound constraint on the control is always active, i.e., $u^{\star}\left(\theta_{2}(\mathbf{w})\right)=L, \forall \mathbf{w} \in \mathscr{H}_{k+1}$.

Therefore, with the assignment in line 2 of Algorithm 1, we obtain an affine control law that is always feasible and also optimal.

When none of the trivial cases holds, we are in case [C4] of $\$ 4.1 .2$. Therefore, we can invoke the results from Lemma 4.3 to argue that the right side of the set $\Delta_{\Gamma^{*}}$ is exactly the set on line 7 of the algorithm, i.e., r-side $\left(\Delta_{\Gamma^{\star}}\right)=\left\{\mathbf{y}_{0}^{\star}, \ldots, \mathbf{y}_{s}^{\star}\right\} \cup\left\{\mathbf{y}_{t}^{\star}\right\} \cup\left\{\mathbf{y}_{r}^{\star}, \ldots, \mathbf{y}_{k}^{\star}\right\}$. In this setting, we can now formulate the first claim about system (33) and its solution.

Lemma 4.4. System (33) is always feasible, and the solution satisfies

(i) $-b_{i} \leq q_{i} \leq 0, \forall i \in\{1, \ldots, k\}$,

(ii) $L \leq q(\mathbf{w}) \leq U, \forall \mathbf{w} \in \mathscr{H}_{k+1}$.

Proof. Note first that system (33) has exactly $k+3$ unknowns, two for the cotangents $K_{U}, K_{L}$ and one for each coefficient $q_{i}, 0 \leq i \leq k$. Also, since $\mid \mathrm{r}$-side $\left(\Delta_{\Gamma^{*}}\right)|\leq| \operatorname{ext}\left(\Delta_{\Gamma^{*}}\right) \mid \leq k+1$, and there are exactly $\mid \mathrm{r}$-side $\left(\Delta_{\Gamma^{*}}\right) \mid$ matching constraints and $k+3-\mid r$-side $\left(\Delta_{\Gamma^{*}}\right) \mid$ alignment constraints, it can be immediately seen that the system is always feasible.

Consider any $q_{i}$ with $i \in\{1, \ldots, s\} \cup\{r+1, \ldots, k\}$. From the matching conditions, we have that $q_{i}=u^{\star}\left(\mathbf{v}_{i}\right)-$ $u^{\star}\left(\mathbf{v}_{i-1}\right)$. By Property $(\mathbf{P 3})$ from $\S 2$, the difference in the values of the optimal control law $u^{\star}(\cdot)$ satisfies

$$
\begin{aligned}
u^{\star}\left(\mathbf{v}_{i}\right)-u^{\star}\left(\mathbf{v}_{i-1}\right) & \stackrel{\text { def }}{=} u^{\star}\left(\theta_{2}\left[\mathbf{v}_{i}\right]\right)-u^{\star}\left(\theta_{2}\left[\mathbf{v}_{i-1}\right]\right), \\
(\text { by }(\mathbf{P 3})) & =-f \cdot\left(\theta_{2}\left[\mathbf{v}_{i}\right]-\theta_{2}\left[\mathbf{v}_{i-1}\right]\right), \\
& \stackrel{(20)}{=}-f \cdot b_{i}, \quad \text { where } f \in[0,1] .
\end{aligned}
$$

Since by (29), $b_{j} \geq 0, \forall j \in\{1, \ldots, k\}$, we immediately obtain $-b_{i} \leq q_{i} \leq 0$, for $i \in\{1, \ldots, s\} \cup\{r+1, \ldots, k\}$.

Now, consider any index $i \in\{s+1, \ldots, t \wedge r\}$, where $t \wedge r \equiv \min (t, r)$. From the conditions in system (33) for alignment below $t$, we have $q_{i}=\left(a_{i}-K_{U} \cdot b_{i}\right) /\left(K_{U}-c\right)$. By summing up all such relations, we obtain

$$
\begin{aligned}
\sum_{i=s+1}^{t \wedge r} q_{i} & =\frac{\sum_{i=s+1}^{t \wedge r} a_{i}-K_{U} \cdot \sum_{i=s+1}^{t \wedge r} b_{i}}{K_{U}-c} \Leftrightarrow \text { (using the matching), } \\
u^{\star}\left(\mathbf{v}_{t \wedge r}\right)-u^{\star}\left(\mathbf{v}_{s}\right) & =\frac{\sum_{i=s+1}^{t \wedge r} a_{i}-K_{U} \cdot \sum_{i=s+1}^{t \wedge r} b_{i}}{K_{U}-c} \Leftrightarrow \\
K_{U} & =\frac{\sum_{i=s+1}^{t \wedge r} a_{i}+c \cdot\left(u^{\star}\left(\mathbf{v}_{t \wedge r}\right)-u^{\star}\left(\mathbf{v}_{s}\right)\right)}{\sum_{i=s+1}^{t \wedge r} b_{i}+u^{\star}\left(\mathbf{v}_{t \wedge r}\right)-u^{\star}\left(\mathbf{v}_{s}\right)} \\
& =\frac{\left[\sum_{i=0}^{t \wedge r} a_{i}+c \cdot u^{\star}\left(\mathbf{v}_{t \wedge r}\right)\right]-\left[\sum_{i=0}^{s} a_{i}+c \cdot u^{\star}\left(\mathbf{v}_{s}\right)\right]}{\left[\sum_{i=0}^{t \wedge r} b_{i}+u^{\star}\left(\mathbf{v}_{t \wedge r}\right)\right]-\left[\sum_{i=0}^{s} b_{i}+u^{\star}\left(\mathbf{v}_{s}\right)\right]} \\
& \stackrel{(26)}{=} \frac{\gamma_{1}^{\star}\left[\mathbf{y}_{t \wedge r}^{\star}\right]-\gamma_{1}^{\star}\left[\mathbf{y}_{s}^{\star}\right]}{\gamma_{2}^{\star}\left[\mathbf{y}_{t \wedge r}^{\star}\right]-\gamma_{2}^{\star}\left[\mathbf{y}_{s}^{\star}\right]} \stackrel{(21)}{=} \operatorname{cotan}\left(\mathbf{y}_{s}^{\star}, \mathbf{y}_{t \wedge r}^{\star}\right) .
\end{aligned}
$$


In the first step, we have used the fact that both $\mathbf{v}_{s}^{\star}$ and $\mathbf{v}_{\min (t, r)}^{\star}$ are matched, hence the intermediate coefficients $q_{i}$ must sum to exactly the difference of the values of $u^{\star}(\cdot)$ at $\mathbf{v}_{\min (t, r)}$ and $\mathbf{v}_{s}$, respectively. In this context, we can see that $K_{U}$ is simply the cotangent of the angle formed by the segment $\left[\mathbf{y}_{s}^{\star}, \mathbf{y}_{\min (t, r)}^{\star}\right]$ with the horizontal (i.e., $\gamma_{1}^{\star}$ ) axis. In this case, we can immediately recall result (30) from Lemma 4.3, to argue that $K_{U} \geq\left(a_{s+1}\right) /\left(b_{s+1}\right)$. Combining with (28) and (29), we obtain

$$
K_{U} \geq \frac{a_{s+1}}{b_{s+1}} \geq \stackrel{(29)}{\cdots} \geq \frac{a_{\min (t, r)}}{b_{\min (t, r)}} \geq \frac{a_{t}}{b_{t}} \stackrel{(28)}{>} c .
$$

Therefore we immediately have that for any $i \in\{s+1, \ldots, \min (t, r)\}$,

$$
\left\{\begin{array}{l}
a_{i}-K_{U} \cdot b_{i} \leq 0 \\
K_{U}-c>0
\end{array} \Rightarrow q_{i}=\frac{a_{i}-K_{U} \cdot b_{i}}{K_{U}-c} \leq 0, \quad\left\{\begin{array}{l}
a_{i}-c \cdot b_{i}>0, \\
q_{i}+b_{i}=\frac{a_{i}-c \cdot b_{i}}{K_{U}-c}
\end{array} \Rightarrow q_{i}+b_{i} \geq 0 .\right.\right.
$$

The argument for indices $i \in\{\max (t, s)+1, \ldots, r\}$ proceeds in exactly the same fashion, by recognizing that $K_{L}$ defined in the algorithm is the same as $\operatorname{cotan}\left(\mathbf{y}_{\max (t, s)}^{\star}, \mathbf{y}_{r}^{\star}\right)$, and then applying (30) to argue that $K_{L}<a_{r} / b_{r} \leq$ $\left(a_{\max (t, s)+1}\right) /\left(b_{\max (t, s)+1}\right) \leq\left(a_{t+1}\right) /\left(b_{t+1}\right) \leq c$. This will allow us to use the same reasoning as above, completing the proof of part (i) of the claim.

To prove part (ii), consider any $\mathbf{w} \in \mathscr{H}_{k+1} \stackrel{\text { def }}{=}[0,1]^{k}$. Using part (i), we obtain

$$
\begin{gathered}
q(\mathbf{w}) \stackrel{\text { def }}{=} q_{0}+\sum_{i=1}^{k} q_{i} \cdot w_{i} \leq\left(\text { since } w_{i} \in[0,1], q_{i} \leq 0\right) \leq q_{0} \stackrel{(* *)}{=} u^{\star}\left(\mathbf{v}_{0}\right) \leq U, \\
q(\mathbf{w}) \geq q_{0}+\sum_{i=1}^{k} q_{i} \cdot 1 \stackrel{(* *)}{=} u^{\star}\left(\mathbf{v}_{k}\right) \geq L .
\end{gathered}
$$

Note that in step $(* *)$, we have critically used the result from Lemma 4.3 that, when $\Theta \nsubseteq \mathscr{B}_{L U}$, the points $\mathbf{v}_{0}^{\star}, \mathbf{v}_{k}^{\star}$ are always among the points on the right side of $\Delta_{\Gamma^{*}}$, and therefore we always have the equations $q_{0}=u^{\star}\left(\mathbf{v}_{0}\right), q_{0}+\sum_{i=1}^{k} q_{i}=u^{\star}\left(\mathbf{v}_{k}\right)$ among the matching equations of system (33). For the last arguments, we have simply used the fact that the optimal control law $u^{\star}(\cdot)$ is always feasible, hence $L \leq u^{\star}(\cdot) \leq U$.

This completes our first goal; namely, proving that the affine controller $q(\mathbf{w})$ is always robustly feasible. To complete the construction, we introduce the following final result.

Lemma 4.5. The affine control law $q(\mathbf{w})$ computed in Algorithm 1 verifies Equation (31a).

PRoof. From (32), the affine controller $q(\mathbf{w})$ induces the generators $\mathbf{a}+c \cdot \mathbf{q}$ and $\mathbf{b}+\mathbf{q}$ for the zonogon $\Gamma$. This implies that $\Gamma$ will be the Minkowski sum of the following segments in $\mathbb{R}^{2}$ :

$$
\begin{gathered}
{\left[\begin{array}{c}
a_{1}+c \cdot q_{1} \\
b_{1}+q_{1}
\end{array}\right], \ldots,\left[\begin{array}{c}
a_{s}+c \cdot q_{s} \\
b_{s}+q_{s}
\end{array}\right], \quad\left[\begin{array}{c}
K_{U} \cdot\left(b_{s+1}+q_{s+1}\right) \\
b_{s+1}+q_{s+1}
\end{array}\right], \ldots,\left[\begin{array}{c}
K_{U} \cdot\left(b_{\min (t, r)}+q_{\min (t, r)}\right) \\
b_{\min (t, r)}+q_{\min (t, r)}
\end{array}\right],} \\
{\left[\begin{array}{c}
K_{L} \cdot\left(b_{\max (t, s)+1}+q_{\max (t, s)+1}\right) \\
b_{\max (t, s)+1}+q_{\max (t, s)+1}
\end{array}\right] \ldots,\left[\begin{array}{c}
K_{L} \cdot\left(b_{r}+q_{r}\right) \\
b_{r}+q_{r}
\end{array}\right], \quad\left[\begin{array}{c}
a_{r+1}+c \cdot q_{r+1} \\
b_{r+1}+q_{r+1}
\end{array}\right], \ldots,\left[\begin{array}{c}
a_{k}+c \cdot q_{k} \\
b_{k}+q_{k}
\end{array}\right] .}
\end{gathered}
$$

From Lemma 4.4, we have that $q_{i}+b_{i} \geq 0, \forall i \in\{1, \ldots, k\}$. Therefore, if we consider the points in $\mathbb{R}^{2}$,

$$
\mathbf{y}_{i}=\left(\sum_{j=0}^{i}\left(a_{j}+c \cdot q_{j}\right), \sum_{j=0}^{i}\left(b_{j}+q_{j}\right)\right), \quad \forall i \in\{0, \ldots, k\},
$$

we can make the following simple observations:

- For any vertex $\mathbf{v}_{i} \in \Theta, i \in\{0, \ldots, k\}$, that is matched, i.e., $\mathbf{y}_{i}^{\star} \in \mathrm{r}$-side $\left(\Delta_{\Gamma^{*}}\right)$, if we let $\mathbf{w}_{i}$ represent the unique $^{5}$ vertex of the hypercube $\mathscr{H}_{k}$ projecting onto $\mathbf{v}_{i}$, i.e., $\mathbf{v}_{i}=\left(\theta_{1}\left(\mathbf{w}_{i}\right), \theta_{2}\left(\mathbf{w}_{i}\right)\right)$, then we have

$$
\mathbf{y}_{i} \stackrel{(32)}{=}\left(\gamma_{1}\left(\mathbf{w}_{i}\right), \gamma_{2}\left(\mathbf{w}_{i}\right)\right) \stackrel{(33)}{=}\left(\gamma_{1}^{\star}\left(\mathbf{v}_{i}\right), \gamma_{2}^{\star}\left(\mathbf{v}_{i}\right)\right) \stackrel{(26)}{=} \mathbf{y}_{i}^{\star}
$$

The first equality follows from the definition of the mapping that characterizes the zonogon $\Gamma$. The second equality follows from the fact that for any matched vertex $\mathbf{v}_{i}$, the coordinates in $\Gamma^{\star}$ and $\Gamma$ are exactly the same, and the last equality is simply the definition of the point $\mathbf{y}_{i}^{\star}$.

${ }^{5}$ This vertex is unique because of our standing Assumption 4.2 that the number of vertices in $\Theta$ is $2 k$ (also see part (iv) of Lemma .2 in the appendix). 
- For any vertex $\mathbf{v}_{i} \in \Theta, i \in\{0, \ldots, k\}$, that is not matched, we have

$$
\begin{aligned}
\mathbf{y}_{i} \in\left[\mathbf{y}_{s}, \mathbf{y}_{\min (t, r)}\right], & \forall i \in\{s+1, \ldots, \min (t, r)-1\}, \\
\mathbf{y}_{i} \in\left[\mathbf{y}_{\max (t, s)}, \mathbf{y}_{r}\right], & \forall i \in\{\max (t, s)+1, \ldots, r-1\} .
\end{aligned}
$$

This can be seen directly from (34), since the segments in $\mathbb{R}^{2}$ given by $\left[\mathbf{y}_{s}, \mathbf{y}_{s+1}\right], \ldots,\left[\mathbf{y}_{\min (t, r)-1}, \mathbf{y}_{\min (t, r)}\right]$ are always aligned (with common cotangent, given by $K_{U}$ ), and similarly, the segments $\left[\mathbf{y}_{\max (t, s)}, \mathbf{y}_{\max (t, s)+1}\right], \ldots,\left[\mathbf{y}_{r-1}, \mathbf{y}_{r}\right]$ are also aligned (with common cotangent $K_{L}$ ).

This exactly corresponds to the situation shown earlier in Figure 6. By combining the two observations, it can be seen that the points $\left\{\mathbf{y}_{0}, \mathbf{y}_{1}, \ldots, \mathbf{y}_{s}, \mathbf{y}_{\max (t, s)}, \mathbf{y}_{\min (t, r)}, \mathbf{y}_{r}, \ldots, \mathbf{y}_{k}\right\}$ will satisfy the following properties:

$$
\begin{gathered}
\mathbf{y}_{i}=\mathbf{y}_{i}^{\star}, \quad \forall \mathbf{y}_{i}^{\star} \in \mathrm{r}-\operatorname{side}\left(\Delta_{\Gamma^{\star}}\right), \\
\operatorname{cotan}\left(\mathbf{y}_{0}, \mathbf{y}_{1}\right) \geq \operatorname{cotan}\left(\mathbf{y}_{1}, \mathbf{y}_{2}\right) \geq \cdots \geq \operatorname{cotan}\left(\mathbf{y}_{s-1}, \mathbf{y}_{s}\right) \geq \operatorname{cotan}\left(\mathbf{y}_{s}, \mathbf{y}_{\min (t, r)}\right), \\
\geq \operatorname{cotan}\left(\mathbf{y}_{\max (t, s)}, \mathbf{y}_{r}\right) \geq \operatorname{cotan}\left(\mathbf{y}_{r}, \mathbf{y}_{r+1}\right) \geq \cdots \geq \operatorname{cotan}\left(\mathbf{y}_{k-1}, \mathbf{y}_{k}\right),
\end{gathered}
$$

where the second relation follows simply because the points $\mathbf{y}_{i}^{\star} \in \mathrm{r}$-side $\left(\Delta_{\Gamma^{\star}}\right)$ are extreme points on the right side of a convex hull, and thus satisfy the same string of inequalities. This immediately implies that this set of $\mathbf{y}_{i}$ exactly represent the right side of the zonogon $\Gamma$, which, in turn, implies that $\Gamma \equiv$ z-hull $\left(\left\{\mathbf{y}_{0}^{\star}, \mathbf{y}_{1}^{\star}, \ldots, \mathbf{y}_{s}^{\star}, \mathbf{y}_{\max (t, s)}^{\star}, \mathbf{y}_{\min (t, r)}^{\star}, \mathbf{y}_{r}^{\star}, \mathbf{y}_{r+1}^{\star}, \ldots, \mathbf{y}_{k}^{\star}\right\}\right)$. Then, by Corollary 4.1, the maximum value of problem $(O P T)$ in (23b) is equal to the maximum value of problem $(A F F)$ in (31a), and since the former is always $J_{m M}$, so is that latter.

This concludes the construction of the affine control law $q(\mathbf{w})$. We have shown that the policy computed by Algorithm 1 satisfies the conditions (31b) and (31a), i.e., is robustly feasible (by Lemma 4.4) and, when used in conjunction with the original convex state costs, preserves the overall optimal min-max value $J_{1}^{\star}\left(x_{1}\right)$ (Lemma 4.5).

4.3. Construction of the affine state cost. Note that we have essentially completed the first part of the induction step. For the second part, we would still need to show how an affine stage cost can be computed, such that constraints (11b) and (11c) are satisfied. We return temporarily to the notation containing time indices, so as to put the current state of the proof into perspective.

In solving problem $(A F F)$ of (31a), we have shown that there exists an affine $q_{k+1}\left(\mathbf{w}_{k+1}\right)$ such that

$$
\begin{aligned}
J_{1}^{\star}\left(x_{1}\right) & =\max _{w_{k+1} \in \mathscr{H}_{k+1}}\left[\theta_{1}\left(\mathbf{w}_{k+1}\right)+c_{k+1} \cdot q_{k+1}\left(\mathbf{w}_{k+1}\right)+g_{k+1}\left(\theta_{2}\left(\mathbf{w}_{k+1}\right)+q_{k+1}\left(\mathbf{w}_{k+1}\right)\right)\right] \\
& \stackrel{(32)}{=} \max _{w_{k+1} \in \mathscr{H}_{k+1}}\left[\gamma_{1}\left(\mathbf{w}_{k+1}\right)+g_{k+1}\left(\gamma_{2}\left(\mathbf{w}_{k+1}\right)\right)\right] .
\end{aligned}
$$

Using the definition of $g_{k+1}(\cdot)$ from $(7 b)$, we can write the above (only retaining the second term) as

$$
\begin{aligned}
J_{1}^{\star}\left(x_{1}\right) & =\max _{w_{k+1} \in \mathscr{H}_{k}}\left[\gamma_{1}\left(\mathbf{w}_{k+1}\right)+\max _{w_{k+1} \in \mathscr{W}_{k+1}}\left[h_{k+2}\left(\gamma_{2}\left(\mathbf{w}_{k+1}\right)+w_{k+2}\right)+J_{k+2}^{\star}\left(\gamma_{2}\left(\mathbf{w}_{k+1}\right)+w_{k+2}\right)\right]\right] \\
& \stackrel{\text { def }}{=} \max _{w_{k+2} \in \mathscr{H}_{k+2}}\left[\tilde{\gamma}_{1}\left(\mathbf{w}_{k+2}\right)+h_{k+2}\left(\tilde{\gamma}_{2}\left(\mathbf{w}_{k+2}\right)\right)+J_{k+2}^{\star}\left(\tilde{\gamma}_{2}\left(\mathbf{w}_{k+2}\right)\right)\right],
\end{aligned}
$$

where $\tilde{\gamma}_{1}\left(\mathbf{w}_{k+2}\right) \stackrel{\text { def }}{=} \gamma_{1}\left(\mathbf{w}_{k+1}\right)$, and $\tilde{\gamma}_{2}\left(\mathbf{w}_{k+2}\right) \stackrel{\text { def }}{=} \gamma_{2}\left(\mathbf{w}_{k+1}\right)+w_{k+2}$. In terms of physical interpretation, $\tilde{\gamma}_{1}$ has the same significance as $\gamma_{1}$, i.e., the cumulative past costs (including the control cost at time $k+1, c \cdot q_{k+1}$ ), while $\tilde{\gamma}_{2}$ represents the state at time $k+2$, i.e., $x_{k+2}$.

Geometrically, it is easy to note that

$$
\widetilde{\Gamma} \stackrel{\text { def }}{=}\left\{\left(\tilde{\gamma}_{1}\left(\mathbf{w}_{k+2}\right), \tilde{\gamma}_{2}\left(\mathbf{w}_{k+2}\right)\right): \mathbf{w}_{k+2} \in \mathscr{H}_{k+2}\right\}
$$

represents yet another zonogon, obtained by projecting a hyperrectangle $\mathscr{H}_{k+2} \subset \mathbb{R}^{k+1}$ into $\mathbb{R}^{2}$. It has a particular shape relative to the zonogon $\Gamma=\left(\gamma_{1}, \gamma_{2}\right)$, since the generators of $\widetilde{\Gamma}$ are simply obtained by appending a 0 and a 1 , respectively, to the generators of $\Gamma$, which implies that $\widetilde{\Gamma}$ is the convex hull of two translated copies of $\Gamma$, where the translation occurs on the $\tilde{\gamma}_{2}$ axis. As it turns out, this fact will bear little importance for the discussion to follow, so we include it here only for completeness. 
In this context, the problem we would like to solve is to replace the convex function $h_{k+2}\left(\tilde{\gamma}_{2}\left(\mathbf{w}_{k+2}\right)\right)$ with an affine function $z_{k+2}\left(\mathbf{w}_{k+2}\right)$ such that the analogues of conditions (11b) and (11c) are obeyed:

$$
\begin{gathered}
z_{k+2}\left(\mathbf{w}_{k+2}\right) \geq h_{k+2}\left(\tilde{\gamma}_{2}\left(\mathbf{w}_{k+2}\right)\right), \quad \forall \mathbf{w}_{k+2} \in \mathscr{H}_{k+2}, \\
J_{1}^{\star}\left(x_{1}\right)=\max _{w_{k+2} \in \mathscr{H}_{k+2}}\left[\tilde{\gamma}_{1}\left(\mathbf{w}_{k+2}\right)+z_{k+2}\left(\mathbf{w}_{k+2}\right)+J_{k+2}^{\star}\left(\tilde{\gamma}_{2}\left(\mathbf{w}_{k+2}\right)\right)\right] .
\end{gathered}
$$

We can now switch back to the simplified notation, where the time subscript $k+2$ is removed. Furthermore, to preserve as much of the familiar notation from $\S 4.1 .1$, we denote the generators of zonogon $\tilde{\Gamma}$ by $\mathbf{a}, \mathbf{b} \in \mathbb{R}^{k+1}$, and the coefficients of $z(\mathbf{w})$ by $z_{0}, \mathbf{z}$, so that we have

$$
\tilde{\gamma}_{1}(\mathbf{w})=a_{0}+\mathbf{a}^{\prime} \mathbf{w}, \quad \tilde{\gamma}_{2}(\mathbf{w})=b_{0}+\mathbf{b}^{\prime} \mathbf{w}, \quad z(\mathbf{w})=z_{0}+\mathbf{z}^{\prime} \mathbf{w} .
$$

In perfect analogy to our discussion in $\S 4.1$, we can introduce

$$
\begin{aligned}
\mathbf{v}_{\text {min }} & \stackrel{\text { def }}{=} \arg \max \left\{\tilde{\gamma}_{1}: \tilde{\boldsymbol{\gamma}} \in \arg \min \left\{\xi_{2}^{\prime}: \boldsymbol{\xi}^{\prime} \in \tilde{\Gamma}\right\}\right\} ; \quad \mathbf{v}_{\max } \stackrel{\text { def }}{=} 2 \mathbf{O}-\mathbf{v}_{\min } \quad(\mathbf{O} \text { is the center of } \tilde{\Gamma}) \\
\mathbf{v}_{0} & \left.\stackrel{\text { def }}{=} \mathbf{v}_{\min }, \ldots, \mathbf{v}_{p_{1}} \stackrel{\text { def }}{=} \mathbf{v}_{\max }, \ldots, \mathbf{v}_{2 p_{1}}=\mathbf{v}_{\text {min }} \quad \text { (counterclockwise numbering of the vertices of } \tilde{\Gamma}\right) .
\end{aligned}
$$

Without loss of generality, we work again under Assumptions 4.1, 4.2, and 4.3; i.e., we analyze the case when $\mathscr{H}_{k+2}=[0,1]^{k+1}, p_{1}=k+1$ (the zonogon $\tilde{\Gamma}$ has a maximal number of vertices), and $\mathbf{v}_{i}=[1,1, \ldots, 1,0, \ldots, 0]$ (ones in the first $i$ positions). We also use the same overloaded notation when referring to the map $\tilde{\gamma}: \mathbb{R}^{k+1} \rightarrow \mathbb{R}^{2}$ (i.e., $\tilde{\gamma}_{1,2}(\mathbf{w})$ denote the value assigned by the map to a point $\mathbf{w} \in \mathscr{H}_{k+2}$, while $\tilde{\gamma}_{1,2}\left[\mathbf{v}_{i}\right]$ are the $\tilde{\gamma}_{1,2}$ coordinates of a point $\left.\mathbf{v}_{i} \in \mathbb{R}^{2}\right)$, and we write $h\left(\mathbf{v}_{i}\right)$ and $J^{\star}\left(\mathbf{v}_{i}\right)$ instead of $h\left(\tilde{\gamma}_{2}\left[\mathbf{v}_{i}\right]\right)$ and $J^{\star}\left(\tilde{\gamma}_{2}\left[\mathbf{v}_{i}\right]\right)$, respectively.

With the simplified notation, the goal is to find $z(\mathbf{w})$ such that

$$
\begin{aligned}
z(\mathbf{w}) & \geq h\left(\tilde{\gamma}_{2}(\mathbf{w})\right), \quad \forall \mathbf{w} \in \mathscr{H}_{k+1}, \\
\max _{\left(\tilde{\gamma}_{1}, \tilde{\gamma}_{2}\right) \in \tilde{\Gamma}}\left[\tilde{\gamma}_{1}+h\left(\tilde{\gamma}_{2}\right)+J^{\star}\left(\tilde{\gamma}_{2}\right)\right] & =\max _{w \in \mathscr{F}_{k+1}}\left[\tilde{\gamma}_{1}(\mathbf{w})+z(\mathbf{w})+J^{\star}\left(\tilde{\gamma}_{2}(\mathbf{w})\right)\right] .
\end{aligned}
$$

In (38b), the maximization on the left corresponds to the problem solved by the uncertainties $\mathbf{w}$ when the original convex state cost $h\left(\tilde{\gamma}_{2}\right)$ is incurred. As such, the result of the maximization is always exactly equal to $J_{1}^{\star}\left(x_{1}\right)$, the overall min-max value. The maximization on the right corresponds to the problem solved by the uncertainties when the affine cost $z(\mathbf{w})$ is incurred instead of the convex cost. Requiring that the two optimal values be equal thus amounts to preserving the overall min-max value.

Since $h$ and $J^{\star}$ are convex (see Property (P2) in §2), we can immediately use Lemma 4.1 to conclude that the optimal value in the left maximization problem in (38b) is reached at one of the vertices $\mathbf{v}_{0}, \ldots, \mathbf{v}_{k+1}$ found in $\mathrm{r}$-side $(\widetilde{\Gamma})$. Therefore, by introducing the points

$$
\mathbf{y}_{i}^{\star} \stackrel{\text { def }}{=}\left(\tilde{\gamma}_{1}\left[\mathbf{v}_{i}\right]+h\left(\mathbf{v}_{i}\right), \tilde{\gamma}_{2}\left[\mathbf{v}_{i}\right]\right), \quad \forall i \in\{0, \ldots, k+1\},
$$

we can immediately conclude the following result.

Lemma 4.6. The maximum in problem

$$
\begin{gathered}
\max _{\left(\pi_{1}, \pi_{2}\right) \in \Pi^{\star}}\left[\pi_{1}+J^{\star}\left(\pi_{2}\right)\right], \\
\Pi^{\star} \stackrel{\text { def }}{=}\left\{\left(\pi_{1}^{\star}, \pi_{2}^{\star}\right) \in \mathbb{R}^{2}: \pi_{1}^{\star} \stackrel{\text { def }}{=} \tilde{\gamma}_{1}+h\left(\tilde{\gamma}_{2}\right), \pi_{2}^{\star} \stackrel{\text { def }}{=} \tilde{\gamma}_{2},\left(\tilde{\gamma}_{1}, \tilde{\gamma}_{2}\right) \in \tilde{\Gamma}\right\},
\end{gathered}
$$

is reached on the right side of

$$
\Delta_{\Pi^{\star}} \stackrel{\text { def }}{=} \operatorname{conv}\left(\left\{\mathbf{y}_{0}^{\star}, \ldots, \mathbf{y}_{k+1}^{\star}\right\}\right)
$$

Proof. The result is analogous to Lemma 4.2, and the proof is a rehashing of similar ideas. In particular, first note that problem $(O P T)$ is a rewriting of the left maximization in (38b). Therefore, since the maximum of the latter problem is reached at the vertices $\mathbf{v}_{i}, i \in\{0, \ldots, k+1\}$, of zonogon $\widetilde{\Gamma}$, by the definition (39) of the points $\mathbf{y}_{i}^{\star}$, we can conclude that the maximum in problem $(O P T)$ must be reached on the set $\left\{\mathbf{y}_{0}^{\star}, \ldots, \mathbf{y}_{k+1}^{\star}\right\}$. Noting that the function maximized in $(O P T)$ is convex, this set of points can be replaced with its convex hull, $\Delta_{\Pi^{*}}$, without affecting the result. Furthermore, since $J^{\star}$ is convex, by applying the results in Corollary 4.1, and replacing the set by the right side of its convex hull, r-side $\left(\Delta_{\Pi^{*}}\right)$, the optimal value remains unchanged. 
The significance of the new variables $\pi_{1,2}^{\star}$ is as follows. $\pi_{1}^{\star}$ represents the cumulative past stage costs, plus the true (i.e., ideal) convex cost as stage $k+1$, while $\pi_{2}^{\star}$, just like $\tilde{\gamma}_{2}$, stands for the state at the next time step, $x_{k+2}$.

Continuing the analogy with $\S 4.2$, the right optimization in $(38 \mathrm{~b})$ can be rewritten as

$$
\begin{gathered}
\max _{\left(\pi_{1}, \pi_{2}\right) \in \Pi}\left[\pi_{1}+J^{\star}\left(\pi_{2}\right)\right], \\
\text { where } \Pi \stackrel{\text { def }}{=}\left\{\left(\pi_{1}, \pi_{2}\right): \pi_{1}(\mathbf{w}) \stackrel{\text { def }}{=} \tilde{\gamma}_{1}(\mathbf{w})+z(\mathbf{w}), \pi_{2}(\mathbf{w}) \stackrel{\text { def }}{=} \tilde{\gamma}_{2}(\mathbf{w}), \mathbf{w} \in \mathscr{H}_{k+2}\right\} .
\end{gathered}
$$

To examine the maximum in problem $(A F F)$, we remark that its feasible set, $\Pi \subset \mathbb{R}^{2}$, also represents a zonogon, with generators given by $\mathbf{a}+\mathbf{z}$ and $\mathbf{b}$, respectively. Therefore, by Lemma 4.1, the maximum of problem (AFF) is reached at one of the vertices on r-side( $\Pi)$.

Using the same key idea from the construction of the affine control law, we now argue that, if the coefficients of the affine cost, $z_{i}$, were computed in such a way that $\Pi$ represented the zonogon hull of the set of points $\left\{\mathbf{y}_{0}^{\star}, \ldots, \mathbf{y}_{k+1}^{\star}\right\}$, then (by Corollary 4.1 ), the maximum value of problem $(A F F)$ would be the same as the maximum value of problem $(O P T)$.

To this end, we introduce the following procedure for computing the affine cost $z(\mathbf{w})$.

Algorithm 2 (Compute affine stage cost $z(\mathbf{w})$ )

Require: $\tilde{\gamma}_{1}(\mathbf{w}), \tilde{\gamma}_{2}(\mathbf{w}), h(\cdot), J^{\star}(\cdot)$

1. Apply the mapping (39) to obtain $\mathbf{v}_{i}^{\star}, \forall i \in\{0, \ldots, k+1\}$.

2. Compute the set $\Delta_{\Pi^{\star}}=\operatorname{conv}\left(\left\{\mathbf{y}_{0}^{\star}, \ldots, \mathbf{y}_{k+1}^{\star}\right\}\right)$.

3. Let r-side $\left(\Delta_{\Pi^{\star}}\right) \stackrel{\text { def }}{=}\left\{\mathbf{y}_{s(1)}^{\star}, \ldots, \mathbf{y}_{s(n)}^{\star}\right\}$, where $s(1) \leq s(2) \leq \cdots \leq s(n) \in\{0, \ldots, k+1\}$ are the sorted indices of points on the right side of $\Delta_{\Pi^{\star}}$.

4. Solve the following system for $z_{j},(j \in\{0, \ldots, k+1\})$, and $K_{s(i)},(i \in\{2, \ldots, n\})$ :

$$
\begin{cases}z_{0}+z_{1}+\cdots+z_{s(i)}=h\left(\mathbf{v}_{s(i)}\right), & \forall \mathbf{y}_{s(i)}^{\star} \in \mathrm{r}-\operatorname{side}\left(\Delta_{\Pi^{\star}}\right) \quad(\text { matching) } \\ \frac{z_{j}+a_{j}}{b_{j}}=K_{s(i)}, & \forall j \in\{s(i-1)+1, \ldots, s(i)\}, \quad \forall i \in\{2, \ldots, n\}, \quad \text { (alignment). }\end{cases}
$$

5. $\operatorname{Return} z(\mathbf{w})=z_{0}+\sum_{i=1}^{k+1} z_{i} \cdot w_{i}$.

To visualize how the algorithm is working, an extended example is included in Figure 7.

The intuition behind the construction is the same as that presented in $\$ 4.2$. In particular, the matching constraints in system (43) ensure that for any vertex $\mathbf{w}$ of the hypercube $\mathscr{H}_{k+2}$ that corresponds to a potential maximizer in problem $(O P T)$ (through $\mathbf{w} \in \mathscr{H}_{k+2} \stackrel{(36)}{\mapsto} \mathbf{v}_{i} \in \widetilde{\Gamma} \stackrel{(39)}{\mapsto} \mathbf{y}_{i}^{\star} \in \mathrm{r}$-side $\left(\Delta_{\Pi^{\star}}\right)$ ), the value of the affine cost $z(\mathbf{w})$ is equal to the value of the initial convex cost, $h\left(\mathbf{v}_{i}\right)$, implying that the value in problem $(A F F)$ of $(42)$ at $\left(\pi_{1}(\mathbf{w}), \pi_{2}(\mathbf{w})\right)$ is equal to the value in problem $(O P T)$ of $(40 \mathrm{a})$ at $\mathbf{y}_{i}^{\star}$. The alignment constraints in system


FIGURE 7. Matching and alignment performed in Algorithm 2. 
(43) ensure that any such matched points, $\left(\pi_{1}(\mathbf{w}), \pi_{2}(\mathbf{w})\right)$, actually correspond to the vertices on the right side of the zonogon $\Pi$, which implies that, as desired, $\Pi \equiv \mathrm{z}$-hull $\left(\left\{\mathbf{y}_{0}^{\star}, \ldots, \mathbf{y}_{k+1}^{\star}\right\}\right)$.

We conclude our preliminary remarks by noting that, similar to the affine construction, system (43) does not directly impose the robust domination constraint (38a). However, as we will soon argue, this result is a by-product of the way the matching and alignment are performed in Algorithm 2.

4.3.1. Affine cost $z(\cdot)$ dominates convex cost $h(\cdot)$ and preserves overall objective. In this section, we prove that the affine cost $z(\mathbf{w})$ computed in Algorithm 2 not only robustly dominates the original convex cost (38a), but also preserves the overall min-max value (38b).

The following lemma summarizes the first main result.

Lemma 4.7. System (43) is always feasible, and the solution $z(\mathbf{w})$ always satisfies Equation (38b).

Proof. We first note that $s(1)=0$ and $s(n)=k+1$, i.e., $\mathbf{y}_{0}^{\star}, \mathbf{y}_{k+1}^{\star} \in \mathrm{r}$-side $\left(\Delta_{\Pi^{\star}}\right)$. To see why that is the case, note that, by (37), $\mathbf{v}_{0}$ will always have the smallest $\tilde{\gamma}_{2}$ coordinate in the zonogon $\tilde{\Gamma}$. Since the transformation (39) yielding $\mathbf{y}_{i}^{\star}$ leaves the second coordinate unchanged, it is always true that

$$
\mathbf{y}_{0}^{\star}=\arg \max \left\{\pi_{1}: \boldsymbol{\pi} \in \arg \min \left\{\pi_{2}^{\prime}: \boldsymbol{\pi}^{\prime} \in\left\{\mathbf{y}_{i}^{\star}, i \in\{0, \ldots, k+1\}\right\}\right\}\right\},
$$

which immediately implies that $\mathbf{y}_{0}^{\star} \in \mathrm{r}$-side $\left(\Delta_{\Pi^{\star}}\right)$. The proof for $\mathbf{y}_{k+1}^{\star}$ follows in an identical matter since $\mathbf{v}_{k+1}$ has the largest $\tilde{\gamma}_{2}$ coordinate in $\tilde{\Gamma}$.

It can then be checked that the following choice of $z_{i}$ always satisfies system (43):

$$
\begin{gathered}
z_{0}=h\left(\mathbf{v}_{0}\right) ; \quad z_{j}=K_{s(i)} \cdot b_{j}-a_{j}, \quad \forall j \in\{s(i-1)+1, \ldots, s(i)\}, \quad \forall i \in\{2, \ldots, n\}, \\
K_{s(i)}=\frac{z_{s(i-1)+1}+\cdots+z_{s(i)}+a_{s(i-1)+1}+\cdots+a_{s(i)}}{b_{s(i-1)+1}+\cdots+b_{s(i)}}=\frac{h\left(v_{s(i)}\right)-h\left(v_{s(i-1)}\right)+a_{s(i-1)+1}+\cdots+a_{s(i)}}{b_{s(i-1)+1}+\cdots+b_{s(i)}} .
\end{gathered}
$$

The proof of the second part of the lemma is analogous to that of Lemma 4.5. To start, consider the feasible set of problem ( $A F F)$ in (42), namely, the zonogon $\Pi$, and note that from (36), its generators are given by $\mathbf{a}+\mathbf{z}$, b:

$$
\left[\begin{array}{c}
\mathbf{a}+\mathbf{z} \\
\mathbf{b}
\end{array}\right]=\left[\begin{array}{cccccc}
a_{1}+z_{1} & \ldots & a_{s(i)}+z_{s(i)} & a_{s(i)+1}+z_{s(i)+1} & \ldots & a_{k+1}+z_{k+1} \\
b_{1} & \ldots & b_{s(1)} & b_{s(1)+1} & \ldots & b_{k+1}
\end{array}\right] .
$$

By introducing the following points in $\mathbb{R}^{2}$,

$$
\mathbf{y}_{i}=\left(\sum_{j=0}^{i}\left(a_{j}+z_{j}\right), \sum_{j=0}^{i} b_{j}\right)
$$

we have the following simple claims:

- For any $\mathbf{v}_{i} \in \mathrm{r}$-side $(\widetilde{\Gamma})$ that is matched, i.e., $\mathbf{y}_{i}^{\star} \in \mathrm{r}$-side $\left(\Delta_{\Pi^{\star}}\right)$, with $\mathbf{w}_{i}=[1,1, \ldots, 1,0, \ldots, 0]$ denoting the unique $^{6}$ vertex of $\mathscr{H}_{k+2}$ satisfying $\left(\tilde{\gamma}_{1}\left(\mathbf{w}_{i}\right), \tilde{\gamma}_{2}\left(\mathbf{w}_{i}\right)\right)=\mathbf{v}_{i}$, we have

$$
\mathbf{y}_{i} \stackrel{(42)}{=}\left(\tilde{\gamma}_{1}\left(\mathbf{w}_{i}\right)+z\left(\mathbf{w}_{i}\right), \tilde{\gamma}_{2}\left(\mathbf{w}_{i}\right)\right) \stackrel{(43)}{=}\left(\tilde{\gamma}_{1}\left[\mathbf{v}_{i}\right]+h\left(\mathbf{v}_{i}\right), \tilde{\gamma}_{2}\left[\mathbf{v}_{i}\right] \stackrel{(39)}{=} \mathbf{y}_{i}^{\star} .\right.
$$

The first equality follows from the definition of the zonogon $\Pi$, the second follows because any $\mathbf{y}_{i}^{\star} \in \mathrm{r}$-side $\left(\Delta_{\Pi^{*}}\right)$ is matched in system (43), and the third equality represents the definition of the points $\mathbf{y}_{i}^{\star}$.

- For any vertex $\mathbf{v}_{j} \in \mathrm{r}$-side $(\tilde{\Gamma})$, which is not matched, i.e., $\mathbf{y}_{j}^{\star} \notin \mathrm{r}$-side $\left(\Delta_{\Pi^{\star}}\right)$, and $s(i)<j<s(i+1)$ for some $i$, we have $\mathbf{y}_{j} \in\left[\mathbf{y}_{s(i)}, \mathbf{y}_{s(i+1)}\right]$. This can be seen by using the alignment conditions in system (43), in conjunction with (44), since the segments in $\mathbb{R}^{2}$ given by $\left[\mathbf{y}_{s(i)}, \mathbf{y}_{s(i)+1}\right],\left[\mathbf{y}_{s(i)+1}, \mathbf{y}_{s(i)+2}\right], \ldots,\left[\mathbf{y}_{s(i+1)-1}, \mathbf{y}_{s(i+1)}\right]$ are always parallel, with common cotangent given by $K_{s(i+1)}$.

For a geometric interpretation, the reader is referred back to Figure 7. Corroborating these results with the fact that $\left\{\mathbf{y}_{s(1)}^{\star}, \ldots, \mathbf{y}_{s(n)}^{\star}\right\}=\operatorname{r-side}\left(\Delta_{\Pi^{\star}}\right)$ must always satisfy

$$
\operatorname{cotan}\left(\mathbf{y}_{s(1)}^{\star}, \mathbf{y}_{s(2)}^{\star}\right) \geq \operatorname{cotan}\left(\mathbf{y}_{s(2)}^{\star}, \mathbf{y}_{s(3)}^{\star}\right) \geq \cdots \geq \operatorname{cotan}\left(\mathbf{y}_{s(n-1)}^{\star}, \mathbf{y}_{s(n)}^{\star}\right),
$$

we immediately obtain that the points $\left\{\mathbf{y}_{s(1)}, \mathbf{y}_{s(2)}, \ldots, \mathbf{y}_{s(n)}\right\}$ exactly represent the right side of the zonogon $\Pi$, which, in turn, implies that $\Pi \equiv \mathrm{z}-\mathrm{hull}\left(\left\{\mathbf{y}_{0}^{\star}, \mathbf{y}_{1}^{\star}, \ldots, \mathbf{y}_{k+1}^{\star}\right\}\right)$. Then, by Corollary 4.1, the maximum value of 
problem $(O P T)$ in (40a) is equal to the maximum value of problem $(A F F)$ in (42), and since the former is always $J_{1}^{\star}\left(x_{1}\right)$, so is that latter.

To complete the second step of the induction, we must only show that the robust domination constraint (38a) is also obeyed:

$$
z(\mathbf{w}) \geq h\left(\tilde{\gamma}_{2}(\mathbf{w})\right) \Leftrightarrow z_{0}+z_{1} \cdot w_{1}+\cdots+z_{k+1} \cdot w_{k+1} \geq h\left(b_{0}+b_{1} \cdot w_{1}+\cdots+b_{k+1} \cdot w_{k+1}\right), \quad \forall \mathbf{w} \in \mathscr{H}_{k+1} .
$$

The following lemma takes us very close to the desired result.

Lemma 4.8. The coefficients for the affine cost $z(\mathbf{w})$ computed in Algorithm 2 always satisfy the following property:

$$
h\left(b_{0}+b_{j(1)}+\cdots+b_{j(m)}\right) \leq z_{0}+z_{j(1)}+\cdots+z_{j(m)}, \quad \forall j(1), \ldots, j(m) \in\{1, \ldots, k+1\}, \quad \forall m \in\{1, \ldots, k+1\} .
$$

Proof. Before proceeding with the proof, we first list several properties related to the construction of the affine cost. We claim that, upon termination, Algorithm 2 always produces a solution to the following system:

$$
\begin{aligned}
& \left\{\begin{array}{l}
z_{0}=h\left(\mathbf{v}_{s(1)}\right) \\
z_{0}+z_{1}+\cdots+z_{s(2)}=h\left(\mathbf{v}_{s(2)}\right) \\
\vdots \quad \vdots \\
z_{0}+z_{1}+\cdots+z_{s(n)}=h\left(\mathbf{v}_{s(n)}\right) \\
\frac{z_{1}+a_{1}}{b_{1}}=\cdots=\frac{z_{s(2)}+a_{s(2)}}{b_{s(2)}}=K_{s(2)} \\
\quad \vdots \quad \\
\frac{z_{s(n-1)+1}+a_{s(n-1)+1}}{b_{s(n-1)+1}}=\cdots=\frac{z_{s(n)}+a_{s(n)}}{b_{s(n)}}=K_{s(n)},
\end{array}\right. \\
& K_{s(2)} \geq \cdots \geq K_{s(n)} \\
& \left\{\begin{array}{l}
\frac{h\left(\mathbf{v}_{j}\right)-h\left(\mathbf{v}_{0}\right)+a_{1}+\cdots+a_{j}}{b_{1}+\cdots+b_{j}} \leq K_{s(2)} \leq \frac{h\left(\mathbf{v}_{s(2)}\right)-h\left(\mathbf{v}_{j}\right)+a_{j+1}+\cdots+a_{s(1)}}{b_{j+1}+\cdots+b_{s(1)}}, \\
\forall j \in\{1, \ldots, s(2)-1\} \\
\vdots \quad \\
\frac{h\left(\mathbf{v}_{j}\right)-h\left(\mathbf{v}_{s(n-1)}\right)+a_{s(n-1)+1}+\cdots+a_{j}}{b_{s(n-1)+1}+\cdots+b_{j}} \leq K_{s(n)} \leq \frac{h\left(\mathbf{v}_{s(n)}\right)-h\left(\mathbf{v}_{j}\right)+a_{j+1}+\cdots+a_{s(n)}}{b_{j+1}+\cdots+b_{s(n)}}, \\
\forall j \in\{s(n-1)+1, \ldots, s(n)-1\} .
\end{array}\right.
\end{aligned}
$$

Let us explain the significance of all the equations. Equation (46) is simply a rewriting of the original system (43), which states that at any vertex $\mathbf{v}_{s(i)}$, the value of the affine function should exactly match the value assigned by the convex function $h(\cdot)$, and the coefficients $z_{i}$ between any two matched vertices should be such that the resulting segments $\left[z_{j}+a_{j}, b_{j}\right]$ are aligned (i.e., the angles they form with the $\pi_{1}$ axis have the same cotangent, specified by $K_{(\cdot)}$ variables). We note that we have explicitly used the fact that $s(1)=0$, which we have shown in the first paragraph of the proof of Lemma 4.7.

Equation (47) is a simple restatement of (45), that the cotangents on the right side of a convex hull must be decreasing. 
Equation (48) is a direct consequence of the fact that $\left\{\mathbf{y}_{s(1)}^{\star}, \mathbf{y}_{s(2)}^{\star}, \ldots, \mathbf{y}_{s(n)}^{\star}\right\}$ represent r-side $\left(\Delta_{\Pi^{\star}}\right)$. To see why that is, consider an arbitrary $j \in\{s(i)+1, \ldots, s(i+1)-1\}$. Since $\mathbf{y}_{j}^{\star} \notin \mathbf{r}$-side $\left(\Delta_{\Pi^{\star}}\right)$, we have

$$
\begin{gathered}
\operatorname{cotan}\left(\mathbf{y}_{s(i)}^{\star}, \mathbf{y}_{j}^{\star}\right) \leq \operatorname{cotan}\left(\mathbf{y}_{j}^{\star}, \mathbf{y}_{s(i+1)}^{\star}\right) \stackrel{(36),(39)}{\Leftrightarrow} \\
\frac{a_{s(i)+1}+\cdots+a_{j}+h\left(\mathbf{v}_{j}\right)-h\left(\mathbf{v}_{s(i)}\right)}{b_{s(i)+1}+\cdots+b_{j}} \leq \frac{a_{j+1}+\cdots+a_{s(i+1)}+h\left(\mathbf{v}_{s(i+1)}\right)-h\left(\mathbf{v}_{j}\right)}{b_{j+1}+\cdots+b_{s(i+1)}} \Leftrightarrow \\
\frac{a_{s(i)+1}+\cdots+a_{j}+h\left(\mathbf{v}_{j}\right)-h\left(\mathbf{v}_{s(i)}\right)}{b_{s(i)+1}+\cdots+b_{j}} \leq K_{s(i+1)} \leq \frac{a_{j+1}+\cdots+a_{s(i+1)}+h\left(\mathbf{v}_{s(i+1)}\right)-h\left(\mathbf{v}_{j}\right)}{b_{j+1}+\cdots+b_{s(i+1)}},
\end{gathered}
$$

where in the last step we have used the mediant inequality ${ }^{7}$ and the fact that, from (46),

$$
K_{s(i+1)}=\operatorname{cotan}\left(\mathbf{y}_{s(i)}^{\star}, \mathbf{y}_{s(i+1)}^{\star}\right)=\frac{a_{s(i)+1}+\cdots+a_{s(i+1)}+h\left(\mathbf{v}_{s(i+1)}\right)-h\left(\mathbf{v}_{s(i)}\right)}{b_{s(i)+1}+\cdots+b_{s(i+1)}}
$$

(refer back to Figure 7 for a geometrical interpretation).

With these observations, we now prove the claim of the lemma. The strategy of the proof will be to use induction on the size of the subsets, $m$. First, we show the property for any subset of indices $j(1), \ldots, j(m) \in$ $\{s(1)=0, \ldots, s(2)\}$, and then extend it to $j(1), \ldots, j(m) \in\{s(i)+1, \ldots, s(i+1)\}$ for any $i$, and then to any subset of $\{1, \ldots, k+1\}$.

The following implications of the conditions (46)-(48) are stated here for convenience, because they are used throughout the rest of the proof:

$$
\begin{gathered}
h\left(\mathbf{v}_{s(1)}\right)=h\left(\mathbf{v}_{0}\right)=z_{0} ; \quad h\left(\mathbf{v}_{s(2)}\right)=z_{0}+z_{1}+\cdots+z_{s(2)} . \\
h\left(\mathbf{v}_{j}\right)-h\left(\mathbf{v}_{0}\right) \leq z_{1}+\cdots+z_{j}, \quad \forall j \in\{1, \ldots, s(2)-1\} . \\
\frac{z_{1}}{b_{1}} \leq \cdots \leq \frac{z_{j}}{b_{j}} \leq \cdots \leq \frac{z_{s(2)}}{b_{s(2)}}, \quad \forall j \in\{1, \ldots, s(2)-1\} .
\end{gathered}
$$

Their proofs are straightforward. Equation (49) follows directly from system (46), and

$$
\begin{gathered}
\frac{h\left(\mathbf{v}_{j}\right)-h\left(\mathbf{v}_{0}\right)+a_{1}+\cdots+a_{j}}{b_{1}+\cdots+b_{j}} \stackrel{(48)}{\leq} K_{s(2)} \stackrel{(46)}{=} \frac{z_{1}+\cdots+z_{j}+a_{1}+\cdots+a_{j}}{b_{1}+\cdots+b_{j}} \Rightarrow(50) \text { true. } \\
\left\{\begin{array}{l}
(46): \frac{a_{1}+z_{1}}{b_{1}}=\cdots=\frac{a_{j}+z_{j}}{b_{j}}=\cdots=\frac{a_{s(2)}+z_{s(2)}}{b_{s(2)}} \\
\Pi \text { zonogon } \Rightarrow \frac{a_{1}}{b_{1}}>\cdots>\frac{a_{j}}{b_{j}}>\cdots>\frac{a_{s(2)}}{b_{s(2)}}
\end{array} \Rightarrow\right. \text { (51) true. }
\end{gathered}
$$

We can now proceed with the proof by checking the induction for $m=1$. We would like to show that

$$
h\left(b_{0}+b_{j}\right) \leq z_{0}+z_{j}, \quad \forall j \in\{1, \ldots, s(2)\} .
$$

Writing $b_{0}+b_{j}$ as

$$
\begin{gathered}
b_{0}+b_{j}=(1-\lambda) \cdot b_{0}+\lambda \cdot\left(b_{0}+\cdots+b_{j}\right), \\
\lambda=\frac{b_{j}}{b_{1}+\cdots+b_{j}},
\end{gathered}
$$

we obtain

$$
\begin{aligned}
h\left(b_{0}+b_{j}\right) & \leq(1-\lambda) \cdot h\left(b_{0}\right)+\lambda \cdot \underbrace{h\left(b_{0}+\cdots+b_{j}\right)}_{\equiv h\left(\mathbf{v}_{j}\right)} \\
& =h\left(\mathbf{v}_{0}\right)+\frac{b_{j}}{b_{1}+\cdots+b_{j}}\left[h\left(\mathbf{v}_{j}\right)-h\left(\mathbf{v}_{0}\right)\right] \leq(\text { by }(49) \text { if } j=s(2) \text { or (50) otherwise }) \\
& \leq z_{0}+\frac{b_{j}}{b_{1}+\cdots+b_{j}}\left(z_{1}+\cdots+z_{j}\right) \leq(\text { by (51) and the mediant inequality }) \\
& \leq z_{0}+z_{j} .
\end{aligned}
$$

${ }^{7}$ If $b, d>0$ and $a / b \leq c / d$, then $a / b \leq(a+c) /(b+d) \leq c / d$. 
Assume the property is true for any subsets of size $m$. Consider a subset $j(1), \ldots, j(m), j(m+1)$, and without loss of generality, let $j(m+1)$ be the largest index. With the convex combination

$$
\begin{aligned}
b^{\star} & \stackrel{\text { def }}{=} b_{0}+b_{j(1)}+\cdots+b_{j(m)}+b_{j(m+1)} \\
& =(1-\lambda) \cdot\left(b_{0}+b_{j(1)}+\cdots+b_{j(m)}\right)+\lambda \cdot\left(b_{0}+b_{1}+\cdots+b_{j(m+1)-1}+b_{j(m+1)}\right),
\end{aligned}
$$

where

$$
\lambda=\frac{b_{j(m+1)}}{\left(b_{1}+b_{2}+\cdots+b_{j(m+1)}\right)-\left(b_{j(1)}+b_{j(2)}+\cdots+b_{j(m)}\right)},
$$

we obtain

$$
\begin{aligned}
h\left(b^{\star}\right) \leq & (1-\lambda) \cdot h\left(b_{0}+b_{j(1)}+\cdots+b_{j(m)}\right)+\lambda \cdot h\left(\mathbf{v}_{i(m+1)}\right) \leq(\text { by induction hypothesis and (49), (50)) } \\
\leq & (1-\lambda) \cdot\left(z_{0}+z_{j(1)}+\cdots+z_{j(m)}\right)+\lambda \cdot\left(z_{0}+z_{1}+\cdots+z_{i(m+1)}\right) \\
= & z_{0}+z_{j(1)}+\cdots+z_{j(m)}+\frac{b_{j(m+1)}}{\left(b_{1}+b_{2}+\cdots+b_{j(m+1)}\right)-\left(b_{j(1)}+b_{j(2)}+\cdots+b_{j(m)}\right)} \\
& \cdot\left[\left(z_{1}+z_{2}+\cdots+z_{j(m+1)}\right)-\left(z_{j(1)}+z_{j(2)}+\cdots+z_{j(m)}\right)\right] \leq(\text { by }(51) \text { and mediant inequality }) \\
\leq & z_{0}+z_{j(1)}+\cdots+z_{j(m)}+z_{j(m+1)} .
\end{aligned}
$$

We claim that the exact same procedure can be repeated for a subset of indices from $\{s(i)+1, \ldots, s(i+1)\}$, for any index $i \in\{1, \ldots, n-1\}$. We would simply be using the adequate inequality from (48), and the statements equivalent to (49)-(51). The following results would be immediate:

$$
\begin{aligned}
h\left(\left(b_{0}+b_{1}+\cdots+b_{s(i)}\right)+b_{j(1)}+\right. & \left.\cdots+b_{j(m)}\right) \leq\left(z_{0}+z_{1}+\cdots+z_{s(i)}\right)+z_{j(1)}+\cdots+z_{j(m)}, \\
& \forall i \in\{1, \ldots, n\}, \quad \forall j(1), \ldots, j(m) \in\{s(i)+1, \ldots, s(i+1)\} .
\end{aligned}
$$

Note that instead of the term $b_{0}$ for the argument of $h(\cdot)$, we would use the complete sum $b_{0}+b_{1}+\cdots+b_{s(i)}$, and similarly, instead of $z_{0}$, we would have the complete sum $z_{0}+z_{1}+\cdots+z_{s(i)}$. With these results, we can make use of the increasing increments property of convex functions

$$
\frac{h\left(x_{1}+\Delta\right)-h\left(x_{1}\right)}{\Delta} \leq \frac{h\left(x_{2}+\Delta\right)-h\left(x_{2}\right)}{\Delta}, \quad \forall \Delta>0, \quad x_{1} \leq x_{2}
$$

to obtain the following result:

$$
\begin{aligned}
& h(b_{0}+\underbrace{b_{j(1)}+\cdots+b_{j(m)}}_{j(\cdot) \in\{1, \ldots, s(2)\}}+\underbrace{b_{i(1)}+\cdots+b_{i(l)}}_{i(\cdot) \in\{s(2)+1, \ldots, s(3)\}})-h\left(b_{0}+b_{j(1)}+\cdots+b_{j(m)}\right) \\
& \quad \leq h(b_{0}+\underbrace{b_{1}+\cdots+b_{s(2)}}_{\text {all indices in }\{1, \ldots, s(2)\}}+b_{i(1)}+\cdots+b_{i(l)})-\underbrace{\leq}_{\stackrel{\text { def } h\left(\mathbf{v}_{s(2)}\right)}{h\left(b_{0}+b_{1}+\cdots+b_{s(2)}\right)} \stackrel{(49),(52)}{\leq}} \\
& \quad \leq\left(z_{0}+z_{1}+\cdots+z_{s(2)}\right)+z_{i(1)}+\cdots+z_{i(l)}-\left(z_{0}+z_{1}+\cdots+z_{s(2)}\right) \\
& \quad=z_{i(1)}+\cdots+z_{i(l)} \Rightarrow \\
& h\left(b_{0}+b_{j(1)}+\cdots+b_{j(m)}+b_{i(1)}+\cdots+b_{i(l)}\right) \leq h\left(b_{0}+b_{j(1)}+\cdots+b_{j(m)}\right)+z_{i(1)}+\cdots+z_{i(l)} \stackrel{(52)}{\leq} \\
& \quad \leq z_{0}+z_{j(1)}+\cdots+z_{j(m)}+z_{i(1)}+\cdots+z_{i(l)} .
\end{aligned}
$$

We showed the property for indices drawn only from the first two intervals, $\{s(1)+1, \ldots, s(2)\}$ and $\{s(2)+1$, $l \ldots, s(3)\}$, but it should be clear how the argument can be immediately extended to any collection of indices, drawn from any intervals. We omit the details for brevity, and conclude that the claim of the lemma is true.

We are now ready for the last major result.

Lemma 4.9. The affine cost $z(\mathbf{w})$ computed by Algorithm 2 always dominates the convex cost $h\left(\tilde{\gamma}_{2}(\mathbf{w})\right)$ :

$$
h\left(b_{0}+\sum_{i=1}^{k+1} b_{i} \cdot w_{i}\right) \leq z_{0}+\sum_{i=1}^{k+1} z_{i} \cdot w_{i}, \quad \forall \mathbf{w} \in \mathscr{H}_{k+1}=[0,1]^{k+1} .
$$


Proof. Note first that the function $f(\mathbf{w}) \stackrel{\text { def }}{=} h\left(b_{0}+\sum_{i=1}^{k+1} b_{i} \cdot w_{i}\right)-\left(z_{0}+\sum_{i=1}^{k+1} z_{i} \cdot w_{i}\right)$ is a convex function of $\mathbf{w}$. Furthermore, the result of Lemma 4.8 can be immediately rewritten as

$$
h\left(b_{0}+\sum_{i=1}^{k+1} b_{i} \cdot w_{i}\right) \leq z_{0}+\sum_{i=1}^{k+1} z_{i} \cdot w_{i}, \quad \forall \mathbf{w} \in\{0,1\}^{k+1} \Leftrightarrow f(\mathbf{w}) \leq 0, \quad \forall \mathbf{w} \in\{0,1\}^{k+1} .
$$

Since the maximum of a convex function on a polytope occurs on the extreme points of the polytope, and $\operatorname{ext}\left(\mathscr{H}_{k+1}\right)=\{0,1\}^{k+1}$, we immediately have that $\max _{w \in \mathscr{H}_{k+1}} f(\mathbf{w})=\max _{w \in\{0,1\}^{k+1}} f(\mathbf{w}) \leq 0$, which completes the proof of the lemma.

We can now conclude the proof of correctness in the construction of the affine stage cost, $z(\mathbf{w})$. With Lemma 4.9, we have that the affine cost always dominates the convex cost $h(\cdot)$, thus condition (38a) is obeyed. Furthermore, from Lemma 4.7, the overall min-max cost remains unchanged even when incurring the affine stage cost, $z(\mathbf{w})$, hence condition (38b) is also true. This completes the construction of the affine cost, and hence also the full step of the induction hypothesis.

4.4. Proof of main theorem. To finalize the current section, we summarize the steps that have lead us to the result, thereby proving the main Theorem 3.1.

Proof OF THEOREM 3.1 In $\S 4.1$, we have verified the induction hypothesis at time $k=1$. With the induction hypothesis assumed true for times $t=1, \ldots, k$, we have listed the initial consequences in Lemma 4.1 and Corollary 4.1 of $\$ 4.1 .1$. By exploring the structure of the optimal control law, $u_{k+1}^{\star}\left(x_{k+1}\right)$ and the optimal value function $J_{k+1}^{\star}\left(x_{k+1}\right)$, in $\S 4.1 .2$, we have finalized the analysis of the induction hypothesis and summarized our findings in Lemmas 4.2 and 4.3.

Section 4.2 then introduced the main construction of the affine control law $q_{k+1}\left(\mathbf{w}_{k+1}\right)$, which was shown to be robustly feasible (Lemma 4.4). Furthermore, in Lemma 4.5, we have shown that, when used in conjuction with the original convex state costs $h_{k+1}\left(x_{k+2}\right)$, this affine control preserves the min-max value of the overall problem.

In $\S 4.3$, we have also introduced an affine stage cost, $z_{k+1}\left(\mathbf{w}_{k+1}\right)$, which, if incurred at time $k+1$, will always preserve the overall min-max value (Lemma 4.7), despite being always larger than the original convex cost, $h_{k+1}\left(x_{k+2}\right)$ (Lemma 4.9).

4.5. Counterexamples for potential extensions. On first sight, one might be tempted to believe that the results in Theorem 3.1 could be immediately extended to more general problems. In particular, one could be tempted to ask one of the following natural questions:

(i) Would both results of Theorem 3.1 (i.e., existence of affine control laws and existence of affine stage costs) hold for a problem that also included linear constraints coupling the controls $u_{t}$ across different time steps? (See Ben-Tal et al. [8] for a situation when this might be of interest.)

(ii) Would both results of Theorem 3.1 hold for multidimensional linear systems? (i.e., problems where $x_{k} \in \mathbb{R}^{d}, \forall k$ with $d \geq 2$.)

(iii) Are affine policies in the disturbances optimal for the two problems above?

(iv) Are affine policies also optimal for stochastic versions of this problem, e.g., for the case where $w_{k}$ is uniformly distributed in $\mathscr{W}_{k}=\left[\underline{w}_{k}, \bar{w}_{k}\right]$, and the goal is to minimize expected costs?

In the rest of the current section, we argue that all of the above questions can be answered negatively. To address the first three, we use the following simple counterexample.

Example 4.1 (Suboptimality of Affine Policies and Affine Cost Relaxations).

$$
\begin{gathered}
T=4, \quad c_{k}=1, h_{k}\left(x_{k+1}\right)=\max \left\{18.5 \cdot x_{k+1},-24 \cdot x_{k+1}\right\}, \quad L_{k}=0, \quad U_{k}=\infty, \quad \forall k \in\{1, \ldots, 4\}, \\
w_{1} \in[-7,0], \quad w_{2} \in[-11,0], \quad w_{3} \in[-8,0], \quad w_{4} \in[-44,0], \\
\sum_{i=1}^{k} u_{i} \leq 10 \cdot k, \quad \forall k \in\{1, \ldots, 4\} .
\end{gathered}
$$

The first two rows describe a one-dimensional problem that fits the conditions of Problem 1 in $\S 1$. The third row corresponds to a coupling constraint for controls at different times, so that the problem fits question (i) above. Furthermore, since the state in such a problem consists of two variables (one for $x_{k}$ and one for $\sum_{i=1}^{k} u_{k}$ ), the example also fits question (ii) above.

The optimal min-max value for Example 4.1 can be found by solving an optimization problem (see Ben-Tal et al. [8]), in which nonanticipatory decisions are computed at all the extreme points of the uncertainty set, 
i.e., for $\left\{\underline{w}_{1}, \bar{w}_{1}\right\} \times\left\{\underline{w}_{2}, \bar{w}_{2}\right\} \times\left\{\underline{w}_{3}, \bar{w}_{3}\right\} \times\left\{\underline{w}_{4}, \bar{w}_{4}\right\}$. The resulting model, which is a large linear program, can be solved to optimality, resulting in a corresponding value of approximately 838.493 for Example 4.1.

To compute the optimal min-max objective obtained by using affine policies $q_{k}\left(\mathbf{w}_{k}\right)$ and incurring affine costs $z_{k}\left(\mathbf{w}_{k+1}\right)$, one can amend the model $(A A R C)$ from $\$ 3$ by including constraints for the cumulative controls (see Ben-Tal et al. [8] for details), and then using (12) to rewrite the resulting model as a linear program. The optimal value of this program for Example 4.1 was approximately 876.057 , resulting in a gap of $4.4 \%$, and thus providing a negative answer to questions (i) and (ii).

To investigate question (iii), we remark that the smallest objective achievable by using affine policies of the type $q_{k}\left(\mathbf{w}_{k}\right)$ can be found by solving another linear optimization problem, having as decision variables the affine coefficients $\left\{q_{k, t}\right\}_{0 \leq t<k \leq T}$, as well as (nonanticipatory) stage cost variables $z_{k}^{\mathbf{w}}$ for every time step $k \in\{1, \ldots, T\}$ and every extreme point $\mathbf{w}$ of the uncertainty set. Solving the resulting linear program for Example 4.1 gave an optimal value of 873.248, so strictly larger than the (true) optimum (838.493) and strictly smaller than the optimal value of the model using both affine control policies and affine stage costs (876.057).

Thus, with question (iii) also answered negatively, we conclude that policies that are affine in the disturbances, $q_{k}\left(\mathbf{w}_{k}\right)$, are, in general, suboptimal for problems with cumulative control constraints or multiple dimensions, and that replacing the convex state costs $h_{k}\left(x_{k+1}\right)$ by (larger) affine costs $z_{k}\left(\mathbf{w}_{k+1}\right)$ would, in general, result in even further deterioration of the objective.

As for question (iv), the following simple example suggests that affine rules are, in general, suboptimal, and that the gap can be arbitrarily large.

Example 4.2 (Suboptimality of Affine Policies in Stochastic Problems).

$$
\begin{array}{ll} 
& J=\mathbb{E}_{w_{1}}\left[\min _{u_{2}\left(w_{1}\right)}\left(u_{2}-w_{1}\right)^{2}\right], \\
\text { s.t. } & 0 \leq u_{2} \leq \frac{1}{K} \\
& w_{1} \sim \text { Uniform }[0,1], \\
& K \in(1,3) \text { fixed and known. }
\end{array}
$$

From the convexity of the problem, it is easy to see that the optimal policy is

$$
u_{2}^{\star}\left(w_{1}\right)= \begin{cases}w_{1} & \text { if } 0 \leq w_{1} \leq \frac{1}{K} \\ \frac{1}{K} & \text { otherwise, }\end{cases}
$$

which results in an objective $J^{\star}=(K-1)^{3} /\left(3 K^{3}\right)$. It can also be easily shown that the optimal objective achievable under affine rules (that satisfy the constraint almost surely) is $J^{A F F}=(K-1)^{2} /\left(4 K^{2}\right)$, for $u_{2}^{A F F}\left(w_{1}\right)=$ $((3-K) /(2 K)) w_{1}+(K-1) /(2 K)$. In particular, note that the relative optimality gap, $\left(J^{A F F}-J^{\star}\right) / J^{\star}=$ $(4-K) /(4(K-1))$, can be made arbitrarily large by taking $K \searrow 1$.

5. An application in inventory management. In this section, we would like to explore our results in connection with the classical inventory problem mentioned in Example 1.1. This example was originally considered by Ben-Tal et al. [8], in the context of a more general model: a retailer-supplier with flexible commitment contracts problem. We first describe the problem in detail, and then draw a connection with our results.

The setting is the following: consider a single-product, single-echelon, multiperiod supply chain, in which inventories are managed periodically over a planning horizon of $T$ periods. The unknown demands $w_{t}$ from customers arrive at the (unique) echelon, henceforth referred to as the retailer, and are satisfied from the onhand inventory, denoted by $x_{t}$ at the beginning of period $t$. The retailer can replenish the inventory by placing orders $u_{t}$, at the beginning of each period $t$, for a cost of $c_{t}$ per unit of product. These orders are immediately available, i.e., there is no lead time in the system, but there are capacities on how much the retailer can order: $L_{t} \leq u_{t} \leq U_{t}$. After the demand $w_{t}$ is realized, the retailer incurs holding costs $H_{t} \cdot \max \left\{0, x_{t}+u_{t}-w_{t}\right\}$ for all the amounts of supply stored on her premises, as well as penalties $B_{t} \cdot \max \left\{w_{t}-x_{t}-u_{t}, 0\right\}$, for any demand that is backlogged.

In the spirit of robust optimization, we assume that the only information available about the demand at time $t$ is that it resides within a certain interval centered around a nominal (or mean) demand $\bar{d}_{t}$, which results in the uncertainty set $W_{t}=\left\{w_{t}:\left\|w_{t}-\bar{d}_{t}\right\| \leq \rho \bar{d}_{t}\right\}$, where $\rho \in[0,1]$ can be interpreted as an uncertainty level. 
As such, if we take the objective function to be minimized as the cost resulting in the worst-case scenario, we immediately obtain an instance of our original Problem 1, with $\alpha_{t}=\beta_{t}=1, \gamma_{t}=-1$, and the convex state costs $h_{t}(\cdot)$ denoting the newsvendor costs, $h_{t}\left(x_{t+1}\right)=H_{t} \cdot \max \left\{x_{t}+u_{t}-w_{t}, 0\right\}+B_{t} \cdot \max \left\{w_{t}-x_{t}-u_{t}, 0\right\}$.

Therefore, the results in Theorem 3.1 are immediately applicable to conclude that no loss of optimality is incurred when we restrict attention to affine order quantities $q_{t}$ that depend on the history of available demands at time $t, q_{t}\left(\mathbf{w}_{t}\right)=q_{t, 0}+\sum_{\tau=1}^{t-1} q_{t, \tau} \cdot w_{\tau}$, and when we replace the newsvendor costs $h_{t}\left(x_{t+1}\right)$ by some (potentially larger) affine costs $z_{t}\left(\mathbf{w}_{t+1}\right)$. The main advantage is that with these substitutions, the problem of finding the optimal affine policies becomes an LP (see the discussion in $\$ 3$ and Ben-Tal et al. [8] for more details).

The more interesting connection with our results comes if we recall the construction in Algorithm 1. In particular, we have the following simple claim.

Proposition 5.1. If the affine orders $q_{t}\left(\mathbf{w}_{t}\right)$ computed in Algorithm 1 are implemented at every time step $t$, and we let $x_{k}\left(\mathbf{w}_{k}\right)=x_{1}+\sum_{t=1}^{k-1}\left(q_{t}\left(\mathbf{w}_{t}\right)-w_{t}\right) \stackrel{\text { def }}{=} x_{t, 0}+\sum_{t=1}^{k-1} x_{k, t} \cdot w_{t}$ denote the affine dependency of the inventory $x_{k}$ on the history of demands, $\mathbf{w}_{k}$, then

(i) If a certain demand $w_{t}$ is fully satisfied by time $k \geq t+1$, i.e., $x_{k, t}=0$, then all the (affine) orders $q_{\tau}$ placed after time $k$ will not depend on $w_{t}$.

(ii) Every demand $w_{t}$ is at most satisfied by the future orders $q_{k}, k \geq t+1$, and the coefficient $q_{k, t}$ represents what fraction of the demand $w_{t}$ is satisfied by the order $q_{k}$.

Proof. To prove the first claim, recall that, in our notation from $\S 4.1 .1, x_{k} \equiv \theta_{2}=b_{0}+\sum_{t=1}^{k-1} b_{t} \cdot w_{t}$. Applying part (i) of Lemma 4.4 in the current setting, ${ }^{8}$ we have that $0 \leq q_{k, t} \leq-x_{k, t}$. Therefore, if $x_{k, t}=0$, then $q_{k, t}=0$, which implies that $x_{k+1, t}=0$. By induction, we immediately get that $q_{\tau, t}=0, \forall \tau \in\{k, \ldots, T\}$.

To prove the second part, note that any given demand, $w_{t}$, initially has an affine coefficient of -1 in the state $x_{t+1}$, i.e., $x_{t+1, t}=-1$. By part (i) of Lemma 4.4, $0 \leq q_{t+1, t} \leq-x_{t+1, t}=1$, so that $q_{t+1, t}$ represents a fraction of the demand $w_{t}$ satisfied by the order $q_{t+1}$. Furthermore, $x_{t+2, t}=x_{t+1, t}+q_{t+1, t} \in[-1,0]$, so by induction we immediately have that $q_{k, t} \in[0,1], \forall k \geq t+1$, and $\sum_{k=t+1}^{T} q_{k, t} \leq 1$.

In view of this result, if we think of $\left\{q_{k}\right\}_{k \geq t+1}$ as future orders that are partially satisfying the demand $w_{t}$, then every future order quantity $q_{k}\left(\mathbf{w}_{k}\right)$ satisfies exactly a fraction of the demand $w_{t}$ (since the coefficient for $w_{t}$ in $q_{k}$ is always in [0,1]), and every demand is at most satisfied by the sequence of orders following after it appears. This interpretation bears some similarity with the unit decomposition approach of Muharremoglu and Tsitsiklis [28], where every unit of supply can be interpreted as satisfying a particular unit of the demand. Here, we are accounting for fractions of the total demand as being satisfied by future order quantities.

6. Conclusions and future directions. We have presented a novel approach for theoretically handling robust multistage decision problems. The method strongly used the connections between the geometrical properties of the feasible sets (zonogons), and the objective functions being optimized, to prune the set of relevant points and derive properties about the optimal policies for the problem. We have also shown an interesting implication of our theoretical results in the context of a classical problem in inventory management.

On a theoretical level, one immediate direction of future research would be to study systems with mixed (polyhedral) constraints, on both state and control at time $t$. Furthermore, we would like to explore the possibility of using the same proof technique in the context of multidimensional problems, as well as for more complicated uncertainty sets $\mathscr{W}$.

Second, we would like to better understand the connections between the matching performed in Algorithm 2 and the properties of convex (and supermodular) functions, and explore extensions of the approach to handle cost functions that are not necessarily convex, as well as nonlinear cost structures for the control $u_{t}$. Another potential area of interest would be to use our analysis tools to quantify the performance of affine policies even in problems where they are known to be suboptimal (such as the one suggested in §4.5). This could potentially lead to fast approximation algorithms with solid theoretical foundations.

On a practical level, we would like to explore potential applications arising in robust portfolio optimization, as well as operations management. Also, we would like to construct a procedure that mimics the behavior of our algorithms, but does not require knowledge of the optimal value functions $J^{\star}(\cdot)$ or optimal controllers $u^{\star}(\cdot)$. One potential idea would be to explore which types of cuts could be added to the linear program (AARC) to ensure that it computes a solution as "close" to the affine controller $q(\mathbf{w})$ as possible.

\footnotetext{
${ }^{8}$ The signs of the inequalities are changed because every disturbance, $w_{t}$, is entering the system dynamics with a coefficient -1 , instead of +1 , as was the case in the discussion from $\$ 4.1 .1$.
} 


\section{Appendix}

A1. DP solution. This section contains a detailed proof for the solution of the DP formulation, initially introduced in $\S 2$. Recall that the problem we would like to solve is the following:

$$
\begin{aligned}
\min _{u_{1}} & {\left[c_{1} u_{1}+\max _{w_{1}}\left[h_{1}\left(x_{2}\right)+\cdots+\min _{u_{k}}\left[c_{k} u_{k}+\max _{w_{k}}\left[h_{k}\left(x_{k+1}\right)+\cdots+\min _{u_{T}}\left[c_{T} u_{T}+\max _{w_{T}} h_{T}\left(x_{T+1}\right)\right] \cdots\right]\right],\right.\right.} \\
\text { s.t. } & x_{k+1}=x_{k}+u_{k}+w_{k}, \\
& L_{k} \leq u_{k} \leq U_{k} \quad \forall k \in\{1,2, \ldots, T\}, \\
& w_{k} \in \mathscr{W}_{k}=\left[\underline{w}_{k}, \bar{w}_{k}\right],
\end{aligned}
$$

which gives rise to the corresponding Bellman recursion:

$$
J_{k}^{\star}\left(x_{k}\right) \stackrel{\text { def }}{=} \min _{L_{k} \leq u_{k} \leq U_{k}}\left[c_{k} u_{k}+\max _{w_{k} \in \mathscr{W}_{k}}\left[h_{k}\left(x_{k}+u_{k}+w_{k}\right)+J_{k+1}^{\star}\left(x_{k}+u_{k}+w_{k}\right)\right]\right] .
$$

According to our definition of running cost and cost-to-go, the cost at $T+1$ is $J_{T+1}^{\star}=0$, which yields the following Bellman recursion at time $T$ :

$$
J_{T}^{\star}\left(x_{T}\right) \stackrel{\text { def }}{=} \min _{L_{T} \leq u_{T} \leq U_{T}}\left[c_{T} \cdot u_{T}+\max _{w_{T} \in \mathscr{W}_{T}} h_{T}\left(x_{T}+u_{T}+w_{T}\right)\right] .
$$

First, consider the inner (maximization) problem. Letting $y_{T} \stackrel{\text { def }}{=} x_{T}+u_{T}$, we obtain

$$
\begin{gathered}
g_{T}\left(y_{T}\right) \stackrel{\text { def }}{=} \max _{w_{T} \in\left[\underline{w}_{T}, \bar{w}_{T}\right]} h_{T}\left(x_{T}+u_{T}+w_{T}\right), \\
\text { (since } \left.h_{T}(\cdot) \text { convex }\right)=\max \left\{h_{T}\left(y_{T}+\underline{w}_{T}\right), h_{T}\left(y_{T}+\bar{w}_{T}\right)\right\} .
\end{gathered}
$$

Note that $g_{T}$ is the maximum of two convex functions of $y_{T}$, hence it is also convex (see Rockafellar [30]). The outer (minimization) problem at time $T$ becomes

$$
\begin{aligned}
J_{T}^{\star}\left(x_{T}\right) & =\min _{L_{T} \leq u_{T}(\cdot) \leq U_{T}} c_{T} \cdot u_{T}+g_{T}\left(x_{T}+u_{T}\right) \\
& =-c_{T} \cdot x_{T}+\min _{L_{T} \leq u_{T}(\cdot) \leq U_{T}}\left[c_{T} \cdot\left(x_{T}+u_{T}\right)+g_{T}\left(x_{T}+u_{T}\right)\right] .
\end{aligned}
$$

For any $x_{T}, c_{T} \cdot\left(x_{T}+u_{T}\right)+g_{T}\left(x_{T}+u_{T}\right)$ is a convex function of its argument $y_{T}=x_{T}+u_{T}$. As such, by defining $y_{T}^{\star}$ to be the minimizer ${ }^{9}$ of the convex function $c_{T} \cdot y+g_{T}(y)$, we obtain that the optimal controller and optimal value function at time $T$ will be

$$
\begin{aligned}
u_{T}^{\star}\left(x_{T}\right) & = \begin{cases}U_{T} & \text { if } x_{T}<y_{T}^{\star}-U_{T}, \\
-x_{T}+y_{T}^{\star} & \text { otherwise, } \\
L_{T} & \text { if } x_{T}>y_{T}^{\star}-L_{T} ;\end{cases} \\
J_{T}^{\star}\left(x_{T}\right) & = \begin{cases}c_{T} \cdot U_{T}+g_{T}\left(x_{T}+U_{T}\right) & \text { if } x_{T}<y_{T}^{\star}-U_{T}, \\
c_{T} \cdot\left(y_{T}^{\star}-x_{T}\right)+g_{T}\left(y_{T}^{\star}\right) & \text { otherwise, } \\
c_{T} \cdot L_{T}+g_{T}\left(x_{T}+L_{T}\right) & \text { if } x_{T}>y_{T}^{\star}-L_{T} .\end{cases}
\end{aligned}
$$

The following properties are immediately obvious:

(i) $u_{T}^{\star}\left(x_{T}\right)$ is piecewise affine (with at most three pieces), continuous, and monotonically decreasing in $x_{T}$.

(ii) $J_{T}^{\star}\left(x_{T}\right)$ is convex, since it represents a partial minimization of a convex function with respect to one of the variables (see Proposition 2.3.6 in Bertsekas et al. [11]).

The results can be immediately extended by induction on $k$.

Lemma .1. The optimal control policy $u_{k}^{\star}\left(x_{k}\right)$ is piecewise affine, with at most three pieces, continuous, and monotonically decreasing in $x_{k}$. The optimal objective function $J_{k}^{\star}\left(x_{k}\right)$ is convex in $x_{t}$.

\footnotetext{
${ }^{9}$ We assume, again, that the minimizer is unique. The results can be extended to a compact set of minimizers, $\left[\underline{y}_{T}, \bar{y}_{T}\right]$.
} 
Proof. The induction is checked at $k=T$. Assume the property is true at $k+1$. Letting $y_{k} \stackrel{\text { def }}{=} x_{k}+u_{k}$, the Bellman recursion at $k$ becomes

$$
\begin{gathered}
J_{k}^{\star}\left(x_{k}\right) \stackrel{\text { def }}{=} \min _{L_{k} \leq u_{k} \leq U_{k}}\left[c_{k} \cdot u_{k}+g_{k}\left(x_{k}+u_{k}\right)\right], \\
g_{k}\left(y_{k}\right) \stackrel{\text { def }}{=} \max _{w_{k} \in \mathscr{W}_{k}}\left[h_{k}\left(y_{k}+w_{k}\right)+J_{k+1}^{\star}\left(y_{k}+w_{k}\right)\right] .
\end{gathered}
$$

Consider first the maximization problem. Since $h_{k}$ is convex and (by the induction hypothesis) $J_{k+1}^{\star}$ is also convex, the maximum will be reached on the boundary of $\mathscr{W}_{k}=\left[\underline{w}_{k}, \bar{w}_{k}\right]$ :

$$
g_{k}\left(y_{k}\right)=\max \left\{h_{k}\left(y_{k}+\underline{w}_{k}\right)+J_{k+1}^{\star}\left(y_{k}+\underline{w}_{k}\right), h_{k}\left(y_{k}+\bar{w}_{k}\right)+J_{k+1}^{\star}\left(y_{k}+\bar{w}_{k}\right)\right\},
$$

and $g_{k}\left(y_{k}\right)$ will also be convex. The minimization problem becomes

$$
\begin{aligned}
J_{k}^{\star}\left(x_{k}\right) & =\min _{L_{k} \leq u_{k} \leq U_{k}}\left[c_{k} \cdot u_{k}+g_{k}\left(x_{k}+u_{k}\right)\right] \\
& =-c_{k} \cdot x_{k}+\min _{L_{k} \leq u_{k} \leq U_{k}}\left[c_{k} \cdot\left(x_{k}+u_{k}\right)+g_{k}\left(x_{k}+u_{k}\right)\right] .
\end{aligned}
$$

Defining, as before, $y_{k}^{\star}$ as the minimizer of $c_{k} \cdot y+g_{k}(y)$, we get

$$
\begin{aligned}
u_{k}^{\star}\left(x_{k}\right) & = \begin{cases}U_{k} & \text { if } x_{k}<y_{k}^{\star}-U_{k}, \\
-x_{k}+y_{k}^{\star} & \text { otherwise, } \\
L_{k} & \text { if } x_{k}>y_{k}^{\star}-L_{k} ;\end{cases} \\
J_{k}^{\star}\left(x_{k}\right) & = \begin{cases}c_{k} \cdot U_{k}+g_{k}\left(x_{k}+U_{k}\right) & \text { if } x_{k}<y_{k}^{\star}-U_{k}, \\
c_{k} \cdot\left(y_{k}^{\star}-x_{k}\right)+g_{k}\left(y_{k}^{\star}\right) & \text { otherwise, } \\
c_{k} \cdot L_{k}+g_{k}\left(x_{k}+L_{k}\right) & \text { if } x_{k}>y_{k}^{\star}-L_{k} .\end{cases}
\end{aligned}
$$

In particular, $u_{k}^{\star}$ will be piecewise affine with three pieces, continuous, and monotonically decreasing, and $J_{k}^{\star}$ will be convex (as the partial minimization of a convex function with respect to one of the variables). A typical example of the optimal control law and the optimal value function is shown in Figure 1.

A2. Zonotopes and zonogons. In this section of the appendix, we would like to outline several useful properties of the main geometrical objects of interest in our exposition; namely, zonotopes. The presentation here parallels that in Chapter 7 of Ziegler [34], to which the interested reader is referred for a much more comprehensive treatment.

Zonotopes are special polytopes that can be viewed in various ways: as projections of hypercubes, as Minkowski sums of line segments, and as sets of bounded linear combinations of vector configurations. Each description gives a different insight into the combinatorics of zonotopes, and there exist some very interesting results that unify the different descriptions under a common theory. For our purposes, it will be sufficient to understand zonotopes under the first two descriptions. In particular, letting $\mathscr{H}_{k}$ denote the $k$-dimensional hypercube, $\mathscr{H}_{k}=\left\{\mathbf{w} \in \mathbb{R}^{k}: 0 \leq w_{i} \leq 1, \forall i\right\}$, we can introduce the following definition.

Definition A.1 (7.13 In Ziegler [34]). A zonotope is the image of a hypercube under an affine projection; that is, a $d$-polytope $Z \subseteq \mathbb{R}^{d}$ of the form

$$
\begin{aligned}
Z=Z(V) & :=V \cdot \mathscr{H}_{k}+\mathbf{z}=\left\{V \mathbf{w}+\mathbf{z}: \mathbf{w} \in \mathscr{H}_{k}\right\} \\
& =\left\{\mathbf{x} \in \mathbb{R}^{d}: \mathbf{x}=\mathbf{z}+\sum_{i=1}^{k} w_{i} \mathbf{v}_{i}, 0 \leq w_{i} \leq 1\right\}
\end{aligned}
$$

for some matrix (vector configuration) $V=\left(\mathbf{v}_{1}, \ldots, \mathbf{v}_{k}\right) \in \mathbb{R}^{d \times k}$ and some $\mathbf{z} \in \mathbb{R}^{d}$.

The rows of the matrix $V$ are often referred to as the generators defining the zonotope. An equivalent description of the zonotope can be obtained by recalling that every $k$-cube $\mathscr{H}_{k}$ is a product of line segments 
$\mathscr{H}_{k}=\mathscr{H}_{1} \times \cdots \times \mathscr{H}_{1}$. Since for a linear operator $\pi$ we always have $\pi\left(\mathscr{H}_{1} \times \cdots \times \mathscr{H}_{1}\right)=\pi\left(\mathscr{H}_{1}\right)+\cdots+\pi\left(\mathscr{H}_{1}\right)$, by considering an affine map given by $\pi(\mathbf{w})=V \mathbf{w}+\mathbf{z}$, it is easy to see that every zonotope is the Minkowski sum of a set of line segments:

$$
Z(V)=\left[0, \mathbf{v}_{1}\right]+\cdots+\left[0, \mathbf{v}_{k}\right]+\mathbf{z} .
$$

For completeness, we remark that there is no loss of generality in regarding a zonotope as a projection from the unit hypercube $\mathscr{H}_{k}$, since any projection from an arbitrary hyperrectangle in $\mathbb{R}^{k}$ can be seen as a projection from the unit hypercube in $\mathbb{R}^{k}$. To see this, consider an arbitrary hyperrectangle in $\mathbb{R}^{k}$ :

$$
\mathscr{W}_{k}=\left[\underline{w}_{1}, \bar{w}_{1}\right] \times\left[\underline{w}_{2}, \bar{w}_{2}\right] \times \cdots \times\left[\underline{w}_{k}, \bar{w}_{k}\right],
$$

and note that with $V \in \mathbb{R}^{d \times k}$ and $\mathbf{a}^{\prime} \in \mathbb{R}^{k}$ denoting the $j$ th row of $V$, the $j$ th component of $Z(V) \stackrel{\text { def }}{=} V \cdot \mathscr{W}_{k}+\mathbf{z}$ can be written as

$$
Z(V)_{j} \stackrel{\text { def }}{=} z_{j}+\sum_{i=1}^{k}\left(a_{i} \cdot w_{i}\right)=\left(z_{j}+\sum_{i=1}^{k} a_{i} \cdot \underline{w}_{i}\right)+\sum_{i=1}^{k} a_{i} \cdot\left(\bar{w}_{i}-\underline{w}_{i}\right) \cdot y_{i}, \quad \text { where } y_{i} \in[0,1], \quad \forall 1 \leq i \leq k .
$$

An example of a subclass of zonotopes are the zonogons, which are all centrally symmetric, two-dimensional $2 p$-gons, arising as the projection of $p$-cubes to the plane. An example is shown in Figure 2. These are the main objects of interest in our treatment, and the following lemma summarizes their most important properties.

Lemma .2. Let $\mathscr{H}_{k}=[0,1]^{k}$ be a $k$-dimensional hypercube, $k \geq 2$. For fixed $\mathbf{a}, \mathbf{b} \in \mathbb{R}^{k}$ and $a_{0}, b_{0} \in \mathbb{R}$, consider the affine transformation

$$
\pi: \mathbb{R}^{k} \rightarrow \mathbb{R}^{2}, \quad \pi(\mathbf{w})=\left[\begin{array}{l}
\mathbf{a}^{\prime} \\
\mathbf{b}^{\prime}
\end{array}\right] \cdot \mathbf{w}+\left[\begin{array}{l}
a_{0} \\
b_{0}
\end{array}\right]
$$

and the zonogon $\Theta \subset \mathbb{R}^{2}$ :

$$
\Theta=\pi\left(\mathscr{H}_{k}\right) \stackrel{\text { def }}{=}\left\{\theta \in \mathbb{R}^{2}: \exists \mathbf{w} \in \mathscr{H}_{k} \text { s.t. } \theta=\pi(\mathbf{w})\right\} .
$$

If we let $\mathscr{V}_{\Theta}$ denote the set of vertices of $\Theta$, then the following properties are true:

(i) $\exists \mathbf{O} \in \Theta$ such that $\Theta$ is symmetric around $\mathbf{O}: \forall \mathbf{x} \in \Theta \Rightarrow 2 \mathbf{O}-\mathbf{x} \in \Theta$.

(ii) $\left|\mathscr{V}_{\Theta}\right|=2 p \leq 2 k$ vertices. Also, $p<k$ if and only if $\exists i \neq j \in\{1, \ldots, k\}$ with $\operatorname{rank}\left(\left[\begin{array}{ll}a_{i} & a_{j} \\ b_{i} & b_{j}\end{array}\right]\right)<2$.

(iii) If we number the vertices of $\mathscr{V}_{\Theta}$ in cyclic order:

$$
\mathscr{V}_{\Theta}=\left(\mathbf{v}_{0}, \ldots, \mathbf{v}_{i}, \mathbf{v}_{i+1}, \ldots, \mathbf{v}_{2 p-1}\right) \quad\left(\mathbf{v}_{2 p+i} \stackrel{\text { def }}{=} \mathbf{v}_{(2 p+i)} \bmod (2 p)\right),
$$

then $2 \mathbf{O}-\mathbf{v}_{i}=\mathbf{v}_{i+p}$, and we have the following representation for $\Theta$ as a Minkowski sum of line segments:

$$
\begin{aligned}
\Theta & =\mathbf{O}+\left[-\frac{\mathbf{v}_{1}-\mathbf{v}_{0}}{2}, \frac{\mathbf{v}_{1}-\mathbf{v}_{0}}{2}\right]+\cdots+\left[-\frac{\mathbf{v}_{p}-\mathbf{v}_{p-1}}{2}, \frac{\mathbf{v}_{p}-\mathbf{v}_{p-1}}{2}\right] \\
& \stackrel{\text { def }}{=} \mathbf{O}+\sum_{i=1}^{p} \lambda_{i} \cdot \frac{\mathbf{v}_{i}-\mathbf{v}_{i-1}}{2}, \quad-1 \leq \lambda_{i} \leq 1 .
\end{aligned}
$$

(iv) If $\exists \mathbf{w}_{1}, \mathbf{w}_{2} \in \mathscr{H}_{k}$ such that $\mathbf{v}_{1} \stackrel{\text { def }}{=} \pi\left(\mathbf{w}_{1}\right)=\mathbf{v}_{2} \stackrel{\text { def }}{=} \pi\left(\mathbf{w}_{2}\right)$ and $\mathbf{v}_{1,2} \in \mathscr{V}_{\Theta}$, then $\exists j \in\{1, \ldots, k\}$ such that $a_{j}=b_{j}=0$.

(v) With the same numbering from (iii) and $k=p$, for any $i \in\{0, \ldots, 2 p-1\}$, the vertices of the hypercube that are projecting to $\mathbf{v}_{i}$ and $\mathbf{v}_{i+1}$, respectively, are adjacent, i.e., they differ only in exactly one component.

PRoof. We will omit a complete proof of the lemma, and will instead simply suggest the main ideas needed for checking the validity of the statements.

For part (i), it is easy to argue that the center of the hypercube, $\mathbf{O}_{\mathscr{H}}=[1 / 2,1 / 2, \ldots, 1 / 2]^{\prime}$, will always project into the center of the zonogon, i.e., $\mathbf{O}=\pi\left(\mathbf{O}_{\mathscr{H}}\right)$. This implies that any zonogon will be centrally symmetric, and will therefore have an even number of vertices.

Part (ii) can be shown by induction on the dimension $k$ of the hypercube, $\mathscr{H}_{k}$. For instance, to prove the first claim, note that the projection of a polytope is simply the convex hull of the projections of the vertices, and therefore projecting a hypercube of dimension $k$ simply amounts to projecting two hypercubes of dimension $k-1$, one for $w_{k}=0$ and another for $w_{k}=1$, and then taking the convex hull of the two resulting polytopes. 
It is easy to see that these two polytopes in $\mathbb{R}^{2}$ are themselves zonogons, and are translated copies of each other (by an amount $\left[a_{k}, b_{k}\right]^{\prime}$ ). Therefore, by the induction hypothesis, they have at most $2(k-1)$ vertices, and taking their convex hull introduces at most two new vertices, for a total of at most $2(k-1)+2=2 k$ vertices. The second claim can be proved in a similar fashion.

One way to prove part (iii) is also by induction on $p$, by taking any pair of opposite (i.e., parallel of the same length) edges and showing that they correspond to a Minkowski summand of the zonogon.

Part (iv) also follows by induction. Using the same argument as for part (ii), note that the only ways to have two distinct vertices of the hypercube $\mathscr{H}_{k}$ (of dimension $k$ ) project onto the same vertex of the zonogon $\Theta$ is to either have this situation happen for one of the two $k-1$ dimensional hypercubes (in which case the induction hypothesis would complete the proof), or to have zero translation between the two zonogons, which could only happen if $a_{k}=b_{k}=0$.

Part (v) follows by using parts (iii) and (iv) and the definition of a zonogon as the Minkowski sum of line segments. In particular, since the difference between two consecutive vertices of the zonogon, $\mathbf{v}_{i}, \mathbf{v}_{i+1}$, for the case $k=p$, is always given by a single column of the projection matrix (i.e., $\left[a_{j}, b_{j}\right]^{\prime}$, for some $j$ ), then the unique vertices of $\mathscr{H}_{k}$ that were projecting onto $\mathbf{v}_{i}$ and $\mathbf{v}_{i+1}$, respectively, must be incidence vectors that differ in exactly one component, i.e., are adjacent on the hypercube $\mathscr{H}_{k}$.

A3. Technical lemmas. This section of the appendix contains a detailed proof for the technical Lemma 4.3 introduced in $\$ 4.1 .2$, which we include below for convenience.

Lemma 4.3. When the zonogon $\Theta$ has a nontrivial intersection with the band $\mathscr{B}_{L U}$ (case [C4]), the convex polygon $\Delta_{\Gamma^{\star}}$ and the set of points on its right side, r-side $\left(\Delta_{\Gamma^{*}}\right)$, satisfy the following properties:

(i) r-side $\left(\Delta_{\Gamma^{\star}}\right)$ is the union of two sequences of consecutive vertices (one starting at $\mathbf{y}_{0}^{\star}$, and one ending at $\left.\mathbf{y}_{k}^{\star}\right)$, and possibly an additional vertex, $\mathbf{y}_{t}^{\star}$,

$$
\mathrm{r} \text {-side }\left(\Delta_{\Gamma^{\star}}\right)=\left\{\mathbf{y}_{0}^{\star}, \mathbf{y}_{1}^{\star}, \ldots, \mathbf{y}_{s}^{\star}\right\} \cup\left\{\mathbf{y}_{t}^{\star}\right\} \cup\left\{\mathbf{y}_{r}^{\star}, \mathbf{y}_{r+1}^{\star} \ldots, \mathbf{y}_{k}^{\star}\right\}, \quad \text { for some } s \leq r \in\{0, \ldots, k\} .
$$

(ii) With $\operatorname{cotan}(\cdot, \cdot)$ given by (21) applied to the $\left(\gamma_{1}^{\star}, \gamma_{2}^{\star}\right)$ coordinates, we have that

$$
\left\{\begin{array}{l}
\operatorname{cotan}\left(\mathbf{y}_{s}^{\star}, \mathbf{y}_{\min (t, r)}^{\star}\right) \geq \frac{a_{s+1}}{b_{s+1}} \quad \text { whenever } t>s, \\
\operatorname{cotan}\left(\mathbf{y}_{\max (t, s)}^{\star}, \mathbf{y}_{r}^{\star}\right) \leq \frac{a_{r}}{b_{r}} \quad \text { whenever } t<r .
\end{array}\right.
$$

Proof of Lemma 4.3. In the following exposition, we use the same notation as introduced in \$4.1.2. Recall that case [C4] on which the lemma is focused corresponds to a nontrivial intersection of the zonotope $\Theta$ with the horizontal band $\mathscr{B}_{L U}$ defined in (27). As suggested in Figure 5, this case can be separated into three subcases, depending on the position of the vertex $\mathbf{v}_{t}$ relative to the band $\mathscr{B}_{L U}$, where the index $t$ is defined in (28). Since the proof of all three cases is essentially identical, we will focus on the more "complicated" situation; namely, when $\mathbf{v}_{t} \in \mathscr{B}_{L U}$. The corresponding arguments for the other two cases should be straightforward.

First, recall that $\Delta_{\Gamma^{\star}}$ is given by (25), i.e., $\Delta_{\Gamma^{\star}}=\operatorname{conv}\left(\left\{\mathbf{y}_{0}^{\star}, \ldots, \mathbf{y}_{k}^{\star}\right\}\right)$, where the points $\mathbf{y}_{i}^{\star}$ are given by (26), which results from applying mapping (24) to $\mathbf{v}_{i} \in \Theta$. From Definition 4.1 of the right side, it can be seen that the points of interest to us; namely, r-side $\left(\Delta_{\Gamma^{*}}\right)$ will be a maximal subset $\left\{\mathbf{y}_{i(1)}^{\star}, \mathbf{y}_{i(2)}^{\star}, \ldots, \mathbf{y}_{i(m)}^{\star}\right\} \subseteq\left\{\mathbf{y}_{0}^{\star}, \ldots, \mathbf{y}_{k}^{\star}\right\}$, satisfying

$$
\left\{\begin{array}{l}
\mathbf{y}_{i(1)}^{\star}=\arg \max \left\{\gamma_{1}: \boldsymbol{\gamma} \in \arg \min \left\{\gamma_{2}^{\prime}: \boldsymbol{\gamma}_{2}^{\prime} \in\left\{\mathbf{y}_{0}^{\star}, \ldots, \mathbf{y}_{k}^{\star}\right\}\right\}\right\}, \\
\mathbf{y}_{i(m)}^{\star}=\arg \max \left\{\gamma_{1}: \boldsymbol{\gamma} \in \arg \max \left\{\gamma_{2}^{\prime}: \boldsymbol{\gamma}_{2}^{\prime} \in\left\{\mathbf{y}_{0}^{\star}, \ldots, \mathbf{y}_{k}^{\star}\right\}\right\}\right\}, \\
\operatorname{cotan}\left(\mathbf{y}_{i(1)}^{\star}, \mathbf{y}_{i(2)}^{\star}\right)>\operatorname{cotan}\left(\mathbf{y}_{i(2)}^{\star}, \mathbf{y}_{i(3)}^{\star}\right)>\cdots>\operatorname{cotan}\left(\mathbf{y}_{i(m-1)}^{\star}, \mathbf{y}_{i(m)}^{\star}\right) .
\end{array}\right.
$$

For the analysis, we find it useful to define the following two indices:

$$
\hat{s} \stackrel{\text { def }}{=} \min \left\{i \in\{0, \ldots, k\}: \theta_{2}\left(\mathbf{v}_{i}\right) \geq y^{\star}-U\right\}, \quad \hat{r} \stackrel{\text { def }}{=} \max \left\{i \in\{0, \ldots, k\}: \theta_{2}\left(\mathbf{v}_{i}\right) \leq y^{\star}-L\right\} .
$$

In particular, $\hat{s}$ is the index of the first vertex of r-side $(\Theta)$ falling inside $\mathscr{B}_{L U}$, and $\hat{r}$ is the index of the last vertex of r-side $(\Theta)$ falling inside $\mathscr{B}_{L U}$. Since we are in the situation when $\mathbf{v}_{t} \in \mathscr{B}_{L U}$, it can be seen that 
$0 \leq \hat{s} \leq t \leq \hat{r} \leq k$, and thus from (28) (the definition of $t$ ) and (29) (typical conditions for the right side of a zonogon):

$$
\frac{a_{1}}{b_{1}}>\cdots>\frac{a_{\hat{s}}}{b_{\hat{s}}}>\cdots>\frac{a_{t}}{b_{t}}>c \geq \frac{a_{t+1}}{b_{t+1}}>\cdots>\frac{a_{\hat{r}}}{b_{\hat{r}}}>\cdots>\frac{a_{k}}{b_{k}} .
$$

With this new notation, we proceed to prove the first result in the claim. First, consider all the vertices

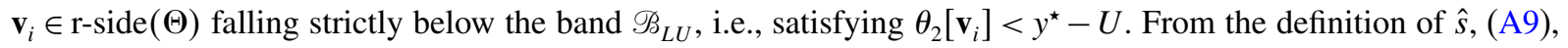
these are exactly $\mathbf{v}_{0}, \ldots, \mathbf{v}_{\hat{s}-1}$, and mapping (24) applied to them will yield $\mathbf{y}_{i}^{\star}=\left(\theta_{1}\left[\mathbf{v}_{i}\right]+c \cdot U, \theta_{2}\left[\mathbf{v}_{i}\right]+U\right)$. In other words, any such points will simply be translated by $(c \cdot U, U)$. Similarly, any points $\mathbf{v}_{i} \in \mathrm{r}$-side $(\Theta)$ falling strictly above the band $\mathscr{B}_{L U}$, i.e., $\theta_{2}\left[\mathbf{v}_{i}\right]>y^{\star}-L$, will be translated by $(c \cdot L, L)$, so that we have

$$
\begin{aligned}
& \mathbf{y}_{i}^{\star}=\mathbf{v}_{i}+(c \cdot U, U), i \in\{0, \ldots, \hat{s}-1\}, \\
& \mathbf{y}_{i}^{\star}=\mathbf{v}_{i}+(c \cdot L, L), \quad i \in\{\hat{r}+1, \ldots, k\},
\end{aligned}
$$

which immediately implies, since $\mathbf{v}_{i} \in \mathrm{r}$-side $(\Theta)$, that

$$
\left\{\begin{array}{l}
\operatorname{cotan}\left(\mathbf{y}_{0}^{\star}, \mathbf{y}_{1}^{\star}\right)>\operatorname{cotan}\left(\mathbf{y}_{1}^{\star}, \mathbf{y}_{2}^{\star}\right)>\cdots>\operatorname{cotan}\left(\mathbf{y}_{\hat{s}-2}^{\star}, \mathbf{y}_{\hat{s}-1}^{\star}\right), \\
\operatorname{cotan}\left(\mathbf{y}_{\hat{r}+1}^{\star}, \mathbf{y}_{\hat{r}+2}^{\star}\right)>\operatorname{cotan}\left(\mathbf{y}_{\hat{r}+2}^{\star}, \mathbf{y}_{\hat{r}+3}^{\star}\right)>\cdots>\operatorname{cotan}\left(\mathbf{y}_{k-1}^{\star}, \mathbf{y}_{k}^{\star}\right) .
\end{array}\right.
$$

For any vertices inside $\mathscr{B}_{L U}$, i.e., $\mathbf{v}_{i} \in \mathrm{r}$-side $(\Theta) \cap \mathscr{B}_{L U}$, mapping (24) will yield

$$
\mathbf{y}_{i}^{\star}=\left(\theta_{1}\left[\mathbf{v}_{i}\right]-c \cdot \theta_{2}\left[\mathbf{v}_{i}\right]+c \cdot y^{\star}, y^{\star}\right), \quad i \in\{\hat{s}, \ldots, t, \ldots, \hat{r}\} ;
$$

that is, they will be mapped into points with the same $\gamma_{2}^{\star}$ coordinates. Furthermore, using (20), it can be seen that $\mathbf{y}_{t}^{\star}$ will have the largest $\gamma_{1}^{\star}$ coordinate among all such $\mathbf{y}_{i}^{\star}$ :

$$
\begin{aligned}
& \gamma_{1}^{\star}\left[\mathbf{y}_{t}^{\star}\right]-\gamma_{1}^{\star}\left[\mathbf{y}_{i}^{\star}\right] \stackrel{\text { def }}{=} \theta_{1}\left[\mathbf{v}_{t}\right]-\theta_{1}\left[\mathbf{v}_{i}\right]-c \cdot\left(\theta_{2}\left[\mathbf{v}_{t}\right]-\theta_{2}\left[\mathbf{v}_{i}\right]\right) \\
& \stackrel{(20)}{=} \begin{cases}\sum_{j=i+1}^{t} a_{j}-c \cdot \sum_{j=i+1}^{t} b_{j} \stackrel{(\mathrm{A} 10)}{\geq} 0, & \text { if } \hat{s} \leq i<t \\
-\sum_{j=t+1}^{i} a_{j}+c \cdot \sum_{j=t+1}^{i} b_{j} \stackrel{(\mathrm{A} 10)}{\geq} 0, & \text { if } t<i \leq \hat{r} .\end{cases}
\end{aligned}
$$

Furthermore, since the mapping (24) yielding $\gamma_{2}^{\star}$ is only a function of $\theta_{2}$, and is monotonic nondecreasing (strictly monotonic increasing outside the band $\mathscr{B}_{L U}$ ), vertices $\mathbf{v}_{0}, \ldots, \mathbf{v}_{k} \in \mathrm{r}$-side $(\Theta)$ will be mapped into points $\mathbf{y}_{0}^{\star}, \ldots, \mathbf{y}_{k}^{\star} \in \gamma^{\star}$ with nondecreasing $\gamma_{2}^{\star}$ coordinates:

$$
\gamma_{2}^{\star}\left[\mathbf{y}_{0}^{\star}\right]<\gamma_{2}^{\star}\left[\mathbf{y}_{1}^{\star}\right]<\cdots<\gamma_{2}^{\star}\left[\mathbf{y}_{\hat{s}-1}^{\star}\right]<y^{\star}=\gamma_{2}^{\star}\left[\mathbf{y}_{\hat{s}}^{\star}\right]=\cdots=\gamma_{2}^{\star}\left[\mathbf{y}_{t}^{\star}\right]=\cdots=\gamma_{2}^{\star}\left[\mathbf{y}_{\hat{r}}^{\star}\right]<\gamma_{2}^{\star}\left[\mathbf{y}_{\hat{r}+1}^{\star}\right]<\cdots<\gamma_{2}^{\star}\left[\mathbf{y}_{k}^{\star}\right] .
$$

Therefore, combining this fact with (A12) and (A14), we can conclude that the points $\mathbf{y}_{i}^{\star}$ satisfying conditions (A8) are none other than

$$
\mathrm{r}-\operatorname{side}\left(\Delta_{\Gamma^{\star}}\right)=\left\{\mathbf{y}_{0}^{\star}, \mathbf{y}_{1}^{\star}, \ldots, \mathbf{y}_{s}^{\star}, \mathbf{y}_{t}^{\star}, \mathbf{y}_{r}^{\star}, \mathbf{y}_{r+1}^{\star}, \mathbf{y}_{k}^{\star}\right\}
$$

where the indices $s$ and $r$ are given as

$$
\begin{aligned}
& s \stackrel{\text { def }}{=}\left\{\begin{array}{l}
\max \left\{i \in\{1, \ldots, \hat{s}-1\}: \operatorname{cotan}\left(\mathbf{y}_{i-1}^{\star}, \mathbf{y}_{i}^{\star}\right)>\operatorname{cotan}\left(\mathbf{y}_{i}^{\star}, \mathbf{y}_{t}^{\star}\right)\right\}, \\
0 \quad \text { if the above condition is never true, }
\end{array}\right. \\
& r \stackrel{\text { def }}{=}\left\{\begin{array}{l}
\min \left\{i \in\{\hat{r}+1, \cdots, k-1\}: \operatorname{cotan}\left(\mathbf{y}_{t}^{\star}, \mathbf{y}_{i}^{\star}\right)>\operatorname{cotan}\left(\mathbf{y}_{i}^{\star}, \mathbf{y}_{i+1}^{\star}\right)\right\}, \\
k \quad \text { if the above condition is never true. }
\end{array}\right.
\end{aligned}
$$

This completes the proof of part (i) of the Lemma. We remark that, for the cases when $\mathbf{v}_{t}$ falls strictly below $\mathscr{B}_{L U}$ or strictly above $\mathscr{B}_{L U}$, one can repeat the exact same reasoning, and immediately argue that the same result would hold. 
To prove the first claim in part (ii), we first recall that, from (A15), if $s<\hat{s}-1$, we must have

$$
\operatorname{cotan}\left(\mathbf{y}_{s}^{\star}, \mathbf{y}_{s+1}^{\star}\right) \leq \operatorname{cotan}\left(\mathbf{y}_{s+1}^{\star}, \mathbf{y}_{t}^{\star}\right),
$$

since otherwise, we would have taken $s+1$ instead of $s$ in (A15). But this immediately implies that

$$
\begin{gathered}
\operatorname{cotan}\left(\mathbf{y}_{s}^{\star}, \mathbf{y}_{s+1}^{\star}\right) \leq \operatorname{cotan}\left(\mathbf{y}_{s+1}^{\star}, \mathbf{y}_{t}^{\star}\right) \stackrel{(21)}{\Leftrightarrow} \frac{\gamma_{1}^{\star}\left[\mathbf{y}_{s+1}^{\star}\right]-\gamma_{1}^{\star}\left[\mathbf{y}_{s}^{\star}\right]}{\gamma_{2}^{\star}\left[\mathbf{y}_{s+1}^{\star}\right]-\gamma_{2}^{\star}\left[\mathbf{y}_{s}^{\star}\right]} \leq \frac{\gamma_{1}^{\star}\left[\mathbf{y}_{t}^{\star}\right]-\gamma_{1}^{\star}\left[\mathbf{y}_{s+1}^{\star}\right]}{\gamma_{2}^{\star}\left[\mathbf{y}_{t}^{\star}\right]-\gamma_{1}^{\star}\left[\mathbf{y}_{s+1}^{\star}\right]} \Rightarrow \text { (mediant inequality) } \\
\frac{\gamma_{1}^{\star}\left[\mathbf{y}_{s+1}^{\star}\right]-\gamma_{1}^{\star}\left[\mathbf{y}_{s}^{\star}\right]}{\gamma_{2}^{\star}\left[\mathbf{y}_{s+1}^{\star}\right]-\gamma_{2}^{\star}\left[\mathbf{y}_{s}^{\star}\right]} \leq \frac{\gamma_{1}^{\star}\left[\mathbf{y}_{t}^{\star}\right]-\gamma_{1}^{\star}\left[\mathbf{y}_{s}^{\star}\right]}{\gamma_{2}^{\star}\left[\mathbf{y}_{t}^{\star}\right]-\gamma_{1}^{\star}\left[\mathbf{y}_{s}^{\star}\right]} \stackrel{(A 11)}{\Leftrightarrow} \frac{a_{s+1}}{b_{s+1}} \leq \operatorname{cotan}\left(\mathbf{y}_{s}^{\star}, \mathbf{y}_{t}^{\star}\right)
\end{gathered}
$$

which is exactly the first claim in part (ii). Thus the only case to discuss is $s=\hat{s}-1$. Since $s \geq 0$, it must be that in this case, there are vertices $\mathbf{v}_{i} \in \mathrm{r}$-side $(\Theta)$ falling strictly below the band $\mathscr{B}_{L U}$. Therefore we can introduce the following point in $\Theta$ :

$$
M \stackrel{\text { def }}{=} \arg \max \left\{\theta_{1}:\left(\theta_{1}, \theta_{2}\right) \in \Theta, \theta_{2}=y^{\star}-U\right\} .
$$

Referring back to Figure 6 in $\S 4.2$, it can be seen that $M$ represents the point with smallest $\theta_{2}$ coordinate in $\mathscr{B}_{L U} \cap \mathrm{r}$-side $(\Theta)$, and $M \in\left[\mathbf{v}_{\hat{s}-1}, \mathbf{v}_{\hat{s}}\right]$. If we let $\left(\theta_{1}[M], \theta_{2}[M]\right)$ denote the coordinates of $M$, then by applying mapping (24) to $M$, the coordinates of the point $\widetilde{M} \in \gamma^{\star}$ are

$$
\widetilde{M}=\left(\theta_{1}[M]+c \cdot U, \theta_{2}[M]+U\right)=\left(\theta_{1}[M]+c \cdot U, y^{\star}\right) .
$$

Furthermore, a similar argument with (A14) can be invoked to show that $\gamma_{1}^{\star}[\widetilde{M}] \leq \gamma_{1}^{\star}\left[\mathbf{y}_{t}^{\star}\right]$. With $s=\hat{s}-1$, we then have

$$
\begin{aligned}
\operatorname{cotan}\left(\mathbf{y}_{s}^{\star}, \mathbf{y}_{t}^{\star}\right) & \stackrel{(21)}{=} \frac{\gamma_{1}^{\star}\left[\mathbf{y}_{t}^{\star}\right]-\gamma_{1}^{\star}\left[\mathbf{y}_{\hat{s}-1}^{\star}\right]}{\gamma_{2}^{\star}\left[\mathbf{y}_{t}^{\star}\right]-\gamma_{2}^{\star}\left[\mathbf{y}_{\hat{s}-1}^{\star}\right]} \geq\left(\text { since } \gamma_{2}^{\star}\left[\mathbf{y}_{t}^{\star}\right]=\gamma_{2}^{\star}[\widetilde{M}]=y^{\star}>\gamma_{2}^{\star}\left[\mathbf{y}_{\hat{s}-1}^{\star}\right]\right) \\
& \geq \frac{\gamma_{1}^{\star}[\widetilde{M}]-\gamma_{1}^{\star}\left[\mathbf{y}_{\hat{s}-1}^{\star}\right]}{\gamma_{2}^{\star}[\widetilde{M}]-\gamma_{2}^{\star}\left[\mathbf{y}_{\hat{s}-1}^{\star}\right]} \\
& \stackrel{\text { (A11),(A17) }}{=} \frac{\theta_{1}[M]-\theta_{1}\left[\mathbf{v}_{\hat{s}-1}\right]}{\theta_{2}[M]-\theta_{2}\left[\mathbf{v}_{\hat{s}-1}\right]}=\left(\text { since } M \in\left[\mathbf{v}_{\hat{s}-1}, \mathbf{v}_{\hat{s}}\right]\right) \\
& =\frac{a_{s+1}}{b_{s+1}}
\end{aligned}
$$

which completes the proof of the first claim in part (ii).

The proof of the second claim in (ii) proceeds in an analogous fashion, by first examining the trivial case $r>\hat{r}+1$ in (A15), and then introducing $N \stackrel{\text { def }}{=} \arg \max \left\{\theta_{1}:\left(\theta_{1}, \theta_{2}\right) \in \Theta, \theta_{2}=y^{\star}-L\right\}$ for the case $r=\hat{r}+1$.

\section{References}

[1] Bemporad, A., F. Borrelli, M. Morari. 2003. Min-max control of constrained uncertain discrete-time linear systems. IEEE Trans. Automatic Control 48(9) 1600-1606.

[2] Ben-Tal, A., A. Nemirovski. 1999. Robust solutions of uncertain linear programs. Oper. Res. Lett. 25 1-13.

[3] Ben-Tal, A., A. Nemirovski. 2002. Robust optimization-Methodology and applications. Math. Program. 92(3) $453-480$.

[4] Ben-Tal, A., S. Boyd, A. Nemirovski. 2005. Control of uncertainty-affected discrete time linear systems via convex programming. Technical report, Minerva Optimization Center, Technion, Haifa, Israel. http://www2.isye.gatech.edu/ nemirovs/FollowUpFinal.pdf.

[5] Ben-Tal, A., S. Boyd, A. Nemirovski. 2006. Extending scope of robust optimization: Comprehensive robust counterparts of uncertain problems. Math. Programming 107(1) 63-89.

[6] Ben-Tal, A., A. Nemirovski, C. Roos. 2002. Robust solutions of uncertain quadratic and conic-quadratic problems. SIAM J. Optim. 13(2) 535-560.

[7] Ben-Tal, A., A. Goryashko, E. Guslitzer, A. Nemirovski. 2004. Adjustable robust solutions of uncertain linear programs. Math. Programming 99(2) 351-376.

[8] Ben-Tal, A., B. Golany, A. Nemirovski, J.-P. Vial. 2005. Retailer-supplier flexible commitments contracts: A robust optimization approach. Manufacturing Service Oper. Management 7(3) 248-271.

[9] Bertsekas, D. P. 2001. Dynamic Programming and Optimal Control. Athena Scientific, Belmont, MA.

[10] Bertsekas, D. P., I. B. Rhodes. 1971. On the minmax reachability of target tubes. Automatica 7 233-247.

[11] Bertsekas, D. P., A. Nedic, A. Ozdaglar. 2003. Convex Analysis and Optimization. Athena Scientific, Nashua, NH.

[12] Bertsimas, D., M. Sim. 2003. Robust discrete optimization and network flows. Math. Programming 98(1-3) 49-71.

[13] Bertsimas, D., M. Sim. 2004. The price of robustness. Oper. Res. 52(1) 35-53. http://or.journal.informs.org/cgi/content/abstract/52/1/35.

[14] Bertsimas, D., D. Pachamanova, M. Sim. 2004. Robust linear optimization under general norms. Oper. Res. Lett. 32(6) 510-516. 
[15] Charnes, A., W. W. Cooper, G. H. Symonds. 1958. Cost horizons and certainty equivalents: An approach to stochastic programming of heating oil. Management Sci. 4(3, April) 235-263.

[16] Chen, X., M. Sim, P. Sun, J. Zhang. 2008. A linear decision-based approximation approach to stochastic programming. Oper. Res. 56(2) 344-357.

[17] Dullerud, G. E., F. Paganini. 2005. A Course in Robust Control Theory. Springer-Verlag, New York.

[18] El-Ghaoui, L., F. Oustry, H. Lebret. 1998. Robust solutions to uncertain semidefinite programs. SIAM J. Optim. 9(1) 33-52.

[19] Fan, M. K. H., A. Tits, J. Doyle. 1991. Robustness in the presence of mixed parametric uncertainty and unmodeled dynamics. IEEE Trans. Automatic Control 36(1) 25-38.

[20] Garstka, S. J., R. J.-B. Wets. 1974. On decision rules in stochastic programming. Math. Programming 7(1) 117-143.

[21] Goulart, P. J., E. C. Kerrigan. 2005. Relationships between affine feedback policies for robust control with constraints. Proc. 16th IFAC World Congress Automatic Control, Prague, Czech Republic.

[22] Grieder, P., P. A. Parrilo, M. Morari. 2003. Robust receding horizon control—Analysis \& synthesis. Proc. 42nd IEEE Conf. Decision Control, Vol. 1. Maui, HI, 941-946.

[23] Kasugai, H., T. Kasegai. 1960. Characteristics of dynamic maximin ordering policy. J. Oper. Res. Soc. Japan 3(1) 11-26.

[24] Kerrigan, E. C., J. M. Maciejowski. 2003. On robust optimization and the optimal control of constrained linear systems with bounded state disturbances. Proc. 2003 Eur. Control Conf., Cambridge, UK.

[25] Kerrigan, E. C., J. M. Maciejowski. 2004. Properties of a new parameterization for the control of constrained systems with disturbances. Proc. 2004 Amer. Control Conf., Vol. 5. 4669-4674. http://ieeexplore.ieee.org/stamp/stamp.jsp?arnumber=01384049.

[26] Kuhn, D., W. Wiesemann, A. Georghiou. 2009. Primal and dual linear decision rules in stochastic and robust optimization. Math. Programming (December) 1-33.

[27] Löfberg, J. 2003. Approximations of closed-loop minimax MPC. Proc. 42nd IEEE Conf. Decision Control, Vol. 2. $1438-1442$.

[28] Muharremoglu, A., J. N. Tsitsiklis. 2008. A single-unit decomposition approach to multiechelon inventory systems. Oper. Res. 56(5) 1089-1103. http://or.journal.informs.org/cgi/content/abstract/56/5/1089.

[29] Nemirovski, A., A. Shapiro. 2005. On complexity of stochastic programming problems. Continuous Optimization: Current Trends and Applications. Springer Verlag, New York, 111-146.

[30] Rockafellar, T. 1970. Convex Analysis. Princeton University Press, Princeton, NJ.

[31] Scarf, H., K. J. Arrow, S. Karlin. 1958. A min-max solution to an inventory problem. Stud. Math. Theory Inventory Production 201-209.

[32] Skaf, J., S. Boyd. 2008. Design of affine controllers via convex optimization. IEEE Trans. Automatic Control. http://www.stanford. edu/ boyd/papers/affine_contr.html.

[33] Zhou, K., J. C. Doyle. 1998. Essentials of Robust Control. Prentice-Hall, Upper Saddle River, NJ.

[34] Ziegler, G. 2003. Lectures on Polytopes, 2nd ed. Springer-Verlag, New York. 